\title{
Fluorous (Trimethylsilyl)ethanol: A New Reagent for Carboxylic Acid Tagging and Protection in Peptide Synthesis
}

\author{
Santos Fustero, ${ }^{+\dagger \neq}$ Amador García Sancho, ${ }^{\ddagger}$ Gema Chiva, ${ }^{\ddagger}$ \\ Juan F. Sanz-Cervera, ${ }^{\dagger, \ddagger}$ Carlos del Pozo, ${ }^{\dagger}$ and José Luis Aceña. ${ }^{\ddagger}$ \\ Departamento de Química Orgánica, Universidad de Valencia, E-46100 Burjassot, Spain, and \\ Laboratorio de Moléculas Orgánicas, Centro de Investigación Príncipe Felipe, E-46013 \\ Valencia, Spain
}

santos.fustero@uv.es

\section{SUPPORTING INFORMATION}

\section{Table of contents:}

General methods

General procedure C

General procedure D

General procedure E

General procedure F

General procedure G

Synthesis of $\mathbf{4}$

Synthesis of $\mathbf{5}$

Synthesis of $\mathbf{6}$

Synthesis of $\mathbf{7}$

S7

Synthesis of $\mathbf{9}$

S7

Synthesis of 12a

S8

Synthesis of $\mathbf{1 2 b}$

S8 
Synthesis of $\mathbf{1 3}$ S9

Synthesis of 14a $\quad$ S9

Synthesis of $\mathbf{1 4 b} \quad$ S9

Synthesis of 15a $\quad \mathrm{S} 10$

Synthesis of 15b $\quad$ S10

Synthesis of $\mathbf{1 5 c} \quad$ S11

Synthesis of 15d $\quad$ S11

$\begin{array}{ll}\text { Synthesis of 15e } & \text { S12 }\end{array}$

$\begin{array}{ll}\text { Synthesis of 16a } & \text { S12 }\end{array}$

Synthesis of $\mathbf{1 6 b} \quad \mathrm{S} 12$

Synthesis of 16c S13

$\begin{array}{ll}\text { Synthesis of 16d } & \text { S13 }\end{array}$

Synthesis of $\mathbf{1 7} \quad \mathrm{S} 14$

$\begin{array}{ll}\text { Synthesis of } \mathbf{1 8} & \text { S14 }\end{array}$

Synthesis of $\mathbf{1 9} \quad$ S15

$\begin{array}{ll}\text { Synthesis of 20a } & \text { S15 }\end{array}$

Synthesis of 20b $\quad$ S16

$\begin{array}{ll}\text { Synthesis of 20c } & \text { S16 }\end{array}$

$\begin{array}{ll}\text { Synthesis of 20d } & \text { S17 }\end{array}$

Synthesis of 1a $\quad$ S18

Synthesis of 1b $\quad \mathrm{S} 18$

$\begin{array}{ll}\text { Synthesis of 1d } & \text { S18 }\end{array}$

NMR spectra of $4 \quad$ S19-S21

NMR spectra of $5 \quad$ S22-S24

$\begin{array}{ll}\text { NMR spectra of } 6 & \text { S25-S27 }\end{array}$

$\begin{array}{lr}\text { NMR spectra of } 7 & \text { S28-S30 }\end{array}$

NMR spectra of 9

NMR spectra of $\mathbf{1 0}$ S33-S35

NMR spectra of $\mathbf{1 1}$ S36-S38

$\begin{array}{ll}\text { NMR spectra of 12a } & \text { S39-S41 }\end{array}$

NMR spectra of 12b $\quad$ S42-S44 
NMR spectra of $\mathbf{1 4 a}$

S45-S46

NMR spectra of $\mathbf{1 4 b}$

S47-S49

NMR spectra of 15a

S50-S52

NMR spectra of $\mathbf{1 5 b}$

S53-S55

NMR spectra of 15c

S56-S58

NMR spectra of 15d

S59-S61

NMR spectra of 15e

S62-S64

NMR spectra of $\mathbf{1 6 a}$

S65-S66

NMR spectra of $\mathbf{1 6 b}$

S67-S68

NMR spectra of $\mathbf{1 6 c}$

S69-S70

NMR spectra of $\mathbf{1 6 d}$

S71-S72

NMR spectra of $\mathbf{1 6 e}$

S73-S74

NMR spectra of $\mathbf{1 6 f}$

S75-S76

NMR spectra of $\mathbf{1 6 g}$

S77-S78

NMR spectra of $\mathbf{1 7}$

S79-S81

NMR spectra of $\mathbf{1 8}$

S82-S83

NMR spectra of 19

S84-S86

NMR spectra of 20a

S87-S89

NMR spectra of $\mathbf{2 0 b}$

S90-S92

NMR spectra of 20c

S93-S95

NMR spectra of $\mathbf{2 0 d}$

S96-S98

NMR spectra of 1c

S99-S101 


\section{General methods.}

All reactions were carried out under argon or nitrogen atmosphere. The solvents were purified prior to use: THF and $\mathrm{Et}_{2} \mathrm{O}$ were distilled from sodium/benzophenone, $\mathrm{CH}_{2} \mathrm{Cl}_{2}$ and $\mathrm{CCl}_{4}$ were distilled from calcium hydride. All reagents were used as received. The reactions were monitored with the aid of thin-layer chromatography (TLC) using $0.25 \mathrm{~mm}$ precoated silica gel plates. Visualization was carried out with UV light and aqueous ceric ammonium molybdate solution or potassium permanganate stain. Flash column chromatography was performed with the indicated solvents on silica gel 60 (particle size 0.040-0.063 mm). Fluorous solid-phase extraction (F-SPE) was performed on cartridges loaded with fluorous silica gel. In a typical purification, the sample was charged on the cartridge and eluted first with $80 \%$ aqueous $\mathrm{MeOH}$ (fluorophobic solvent for eluting non-fluorinated compounds), then with $\mathrm{MeOH}$ (fluorophilic solvent for eluting fluorinated compounds). Melting points are uncorrected. Chemical shifts of NMR spectra are given in ppm $(\delta)$, referenced to the residual proton resonances of the solvents

or fluorotrichloromethane in ${ }^{19}$ F NMR experiments. Coupling constants $(J)$ are given in Hertz $(\mathrm{Hz})$. The letters $\mathrm{m}, \mathrm{s}, \mathrm{d}, \mathrm{t}$, and q stand for multiplet, singlet, doublet, triplet, and quartet, respectively. The letters br indicate that the signal is broad.

\section{General Procedure for the synthesis of fluorous Fmoc-protected amino acids (General Procedure C).}

The corresponding fluorous alcohol (1.0 equiv) was added to a solution of Fmoc-protected amino acid (1.0 equiv) in $\mathrm{CH}_{2} \mathrm{Cl}_{2}(0.2 \mathrm{M})$; subsequently 1-hydroxybenzotriazol (HOBt) (1.2 equiv), diisopropylcarbodiimide (DIC) (1.4 equiv), and dimethylaminopyridine (DMAP) (2.5 equiv) were all added. After stirring at room temperature for $12 \mathrm{~h}$, EtOAc and $\mathrm{H}_{2} \mathrm{O}$ were added and the phases were separated. The organic phase was washed sequentially with $\mathrm{NaHCO}_{3}$ (5\% aq.), citric acid (10\% aq.), and brine, dried over $\mathrm{Na}_{2} \mathrm{SO}_{4}$, and concentrated at reduced pressure. The crude product was purified by means of F-SPE.

\section{General Procedure for the Fmoc deprotection (General Procedure D).}

A solution of the corresponding fluorous Fmoc-protected amino acid (1 equiv) in a 3:1 mixture of piperidine/THF $(0.06 \mathrm{M})$ was stirred at room temperature for $2 \mathrm{~h}$. The crude product was then concentrated at reduced pressure and purified by means of F-SPE. 
General Procedure for the synthesis of fluorous Michael acceptors (General Procedure E). To a cold $\left(0{ }^{\circ} \mathrm{C}\right)$ solution of the corresponding fluorous amine (1 equiv) in sym-collidine (1 equiv), a solution of (trifluoromethyl)acryloyl chloride $2^{1}$ (1.1 equiv) in $\mathrm{CH}_{2} \mathrm{Cl}_{2}(0.04 \mathrm{M})$ was added. After stirring at $0{ }^{\circ} \mathrm{C}$ for $4 \mathrm{~h}$, the reaction was quenched with $\mathrm{NH}_{4} \mathrm{Cl}$ (sat. aq.). The organic phase was washed with $\mathrm{HCl}$ (5\% aq.), dried over $\mathrm{Na}_{2} \mathrm{SO}_{4}$, and concentrated at reduced pressure. The crude product was purified by means of F-SPE.

General Procedure for the aza-Michael addition of $\alpha$-amino esters (General Procedure F).

To a cold $\left(0{ }^{\circ} \mathrm{C}\right)$ solution of the corresponding fluorous Michael acceptor (1 equiv) in $\mathrm{CCl}_{4}(0.04$ M), DABCO (2 equiv) was added followed by the addition of the corresponding $\alpha$-amino ester hydrochloride (1 equiv). After stirring at $0{ }^{\circ} \mathrm{C}$ for $30 \mathrm{~min}$, the crude product was concentrated at reduced pressure and purified with the aid of F-SPE.

\section{General Procedure for the coupling of fluorous amino acids (General Procedure G).}

To a solution of the corresponding fluorous amino acid (1.0 equiv) in DMF (0.1 M), the corresponding Boc-protected amino acid (1.2 equiv), HATU (1.2 equiv), HOAt (1.5 equiv) and sym-collidine (5 equiv) were added. After stirring at room temperature for $18 \mathrm{~h}$, the crude was concentrated at reduced pressure and purified with the aid of F-SPE.

\section{Synthesis of fluorous Fmoc-protected valine, 4.}

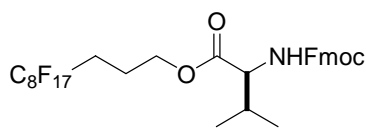

Following General Procedure C, from 3-(perfluorooctyl)propanol 3 (422 mg, $0.88 \mathrm{mmol}$ ) and Fmoc-L-valine (300 mg, $0.88 \mathrm{mmol}$ ), $447 \mathrm{mg}$ of 4 were obtained as a white solid (63\% yield). mp: 97-99 ${ }^{\circ} \mathrm{C} .[\alpha]_{\mathrm{D}}{ }^{25}-0.3\left(\right.$ ( $\left.1.0, \mathrm{CHCl}_{3}\right) .{ }^{1} \mathrm{H}$ NMR $\left(400 \mathrm{MHz}, \mathrm{CDCl}_{3}\right) \delta 0.82$ (d, J=6.8 Hz, 3H), 0.91 (d, $J=6.7$ Hz, 3H), 1.88-1.95 (m, 2H), 2.04-2.15 (m, 3H), 4.11-4.19 (m, 3H), 4.23 (dd, $J=9.1,5.0 \mathrm{~Hz}, 1 \mathrm{H}), 4.28-4.38(\mathrm{~m}, 2 \mathrm{H}), 5.21$ (d, $J=9.0 \mathrm{~Hz}, 1 \mathrm{H}), 7.17-7.69(\mathrm{~m}, 8 \mathrm{H})$; ${ }^{13} \mathrm{C}$ NMR $\left(100 \mathrm{MHz}, \mathrm{CDCl}_{3}\right) \delta 17.5,18.9,19.9\left(\mathrm{t},{ }^{3} J_{\mathrm{CF}}=3.6 \mathrm{~Hz}\right), 27.7\left(\mathrm{t},{ }^{2} J_{\mathrm{CF}}=21.9 \mathrm{~Hz}\right), 31.2,47.1,59.0$, 
63.6, 67.0, 119.9, 119.9, 120.2, 124.3, 125.0, 127.0, 127.7, 129.0, 134.6, 141.3, 143.7, 143.8, 144.4, 156.2, 172.0, (the signals from the $\mathrm{C}_{8} \mathrm{~F}_{17}$ group were obscured due to their low intensity); ${ }^{19} \mathrm{~F}$ NMR $\left(\mathrm{CDCl}_{3}, 282.4 \mathrm{MHz}\right) \delta-81.2\left(\mathrm{t},{ }^{3} J_{\mathrm{FF}}=10.3 \mathrm{~Hz}, 3 \mathrm{~F}\right),-114.8$ (br, 2F), -122.2 (br, 6F), 123.1 (br, 2F), -123.9 (br, 2F), -126.5 (br, 2F). HRMS (FAB): Calcd for $\mathrm{C}_{31} \mathrm{H}_{27} \mathrm{~F}_{17} \mathrm{NO}_{4}\left(\mathrm{M}^{+}+1\right)$ : 800.1668; found: 800.1757 .

\section{Synthesis of fluorous valine, 5 .}

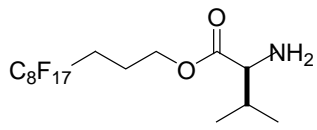

Following General Procedure D, from 4 (109 mg, $0.136 \mathrm{mmol}), 78 \mathrm{mg}$ of 5 were obtained as a white solid (99\% yield). mp: 99-101 ${ }^{\circ} \mathrm{C} .[\alpha]_{\mathrm{D}}{ }^{25}+2.8\left(c \mathrm{c} 0.9, \mathrm{CHCl}_{3}\right) .{ }^{1} \mathrm{H} \mathrm{NMR}\left(\mathrm{CDCl}_{3}, 300 \mathrm{MHz}\right)$ $\delta 0.83$ (d, $J=6.8 \mathrm{~Hz}, 3 \mathrm{H}), 0.91$ (d, $J=6.8 \mathrm{~Hz}, 3 \mathrm{H}), 1.41$ (br, 2H), 1.76-1.82 (m, 1H), 1.89-1.97 (m, 2H), 2.08-2.17 (m, 2H), 3.24 (d, $J=5.1 \mathrm{~Hz}, 1 \mathrm{H}), 4.09-4.18$ (m, 2H); ${ }^{13} \mathrm{C} \mathrm{NMR} \mathrm{(CDCl}, 75.5$ MHz) $\delta 17.0,19.2,20.0\left(\mathrm{t},{ }^{3} J_{\mathrm{CF}}=4.5 \mathrm{~Hz}\right), 27.8\left(\mathrm{t},{ }^{2} J_{\mathrm{CF}}=22.3 \mathrm{~Hz}\right.$ ), 32.1, 59.8, 63.0, 175.3, (the signals from the $\mathrm{C}_{8} \mathrm{~F}_{17}$ group were obscured due to their low intensity); ${ }^{19} \mathrm{~F}$ NMR ( $\mathrm{CDCl}_{3}, 282.4$ MHz) $\delta-81.2\left(t,{ }^{3} J_{\mathrm{FF}}=10.1 \mathrm{~Hz}, 3 \mathrm{~F}\right),-114.8$ (br, 2F), -122.5 (br, 6F), -123.3 (br, 2F), -124.1 (br, 2F), -126.5 (br, 2F). HRMS (FAB): Calcd for $\mathrm{C}_{16} \mathrm{H}_{17} \mathrm{~F}_{17} \mathrm{NO}_{2}\left(\mathrm{M}^{+}+1\right)$ : 578.0987; found: 578.0966 .

\section{Synthesis of fluorous Michael acceptor, 6.}

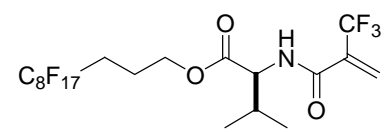

Following General Procedure E, from 5 ( $63 \mathrm{mg}, 0.109 \mathrm{mmol}$ ), $58 \mathrm{mg}$ of 6 were obtained as a colorless oil (75\% yield). $[\alpha]_{D}{ }^{25}-8.2\left(c 0.6, \mathrm{CHCl}_{3}\right) .{ }^{1} \mathrm{H} \mathrm{NMR}\left(\mathrm{CDCl}_{3}, 300 \mathrm{MHz}\right) \delta 0.87$ (d, $J=$ $6.9 \mathrm{~Hz}, 3 \mathrm{H}), 0.91$ (d, $J=6.8 \mathrm{~Hz}, 3 \mathrm{H}), 1.87-2.08$ (m, 2H), 2.11-2.22 (m, 3H), 4.11-4.24 (m, 2H), 4.57 (dd, $J=8.3,4.7 \mathrm{~Hz}, 1 \mathrm{H}), 6.20$ (q, $J=1.1 \mathrm{~Hz}, 1 \mathrm{H}), 6.36$ (d, $J=8.3 \mathrm{~Hz}, 1 \mathrm{H}), 6.50$ (q, $J=1.5$ $\mathrm{Hz}, 1 \mathrm{H}) ;{ }^{13} \mathrm{C}$ NMR $\left(\mathrm{CDCl}_{3}, 75.5 \mathrm{MHz}\right) \delta 17.5,18.8,19.9\left(\mathrm{t},{ }^{3} J_{\mathrm{CF}}=4.0 \mathrm{~Hz}\right), 27.7\left(\mathrm{t},{ }^{2} J_{\mathrm{CF}}=22.4\right.$ $\mathrm{Hz}$ ), 31.2, 57.5, 63.9, 122.1 (q, $\left.{ }^{1} J_{\mathrm{CF}}=273.1 \mathrm{~Hz}\right), 129.9$ (q, $\left.{ }^{3} J_{\mathrm{CF}}=5.7 \mathrm{~Hz}\right), 133.5$ (q, ${ }^{2} J_{\mathrm{CF}}=31.0$ $\mathrm{Hz}$ ), 160.6, 171.1, (the signals from the $\mathrm{C}_{8} \mathrm{~F}_{17}$ group were obscured due to their low intensity);

${ }^{19} \mathrm{~F}$ NMR $\left(\mathrm{CDCl}_{3}, 282.4 \mathrm{MHz}\right) \delta-64.3(\mathrm{~s}, 3 \mathrm{~F}),-81.2\left(\mathrm{t},{ }^{3} \mathrm{JFF}_{\mathrm{FF}}=10.3 \mathrm{~Hz}, 3 \mathrm{~F}\right),-114.8$ (br, 2F), - 
122.2 (br, 6F), -123.2 (br, 2F), -123.9 (br, 2F), -126.6 (br, 2F). HRMS (FAB): Calcd for $\mathrm{C}_{20} \mathrm{H}_{18} \mathrm{~F}_{20} \mathrm{NO}_{3}\left(\mathrm{M}^{+}+1\right)$ : 700.0967; found: 700.1032 .

\section{Synthesis of fluorous retropeptide, 7.}

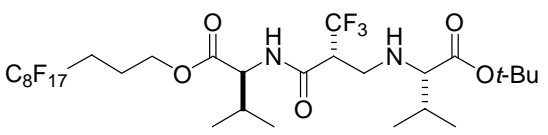

Following General Procedure F, from 6 (158 mg, $0.23 \mathrm{mmol}$ ) and valine tert-butyl ester hydrochloride ( $55 \mathrm{mg}, 0.23 \mathrm{mmol}$ ), $177 \mathrm{mg}$ of 7 were obtained as a colorless oil (90\% yield) in a 27:1 mixture of diastereoisomers as determined from its ${ }^{19} \mathrm{~F}$ NMR spectrum. NMR data for the major diastereoisomer: ${ }^{1} \mathrm{H}$ NMR $\left(\mathrm{CDCl}_{3}, 300 \mathrm{MHz}\right) \delta 0.82$ (d, $\left.J=5.1 \mathrm{~Hz}, 3 \mathrm{H}\right), 0.86$ (d, $J=6.8$ Hz, 3H), 0.87 (d, $J=6.8 \mathrm{~Hz}, 3 \mathrm{H}$ ), 0.92 (d, $J=6.8 \mathrm{~Hz}, 3 \mathrm{H}$ ), 1.40 (s, 9H), 1.82-1.96 (m, 4H), 2.052.19 (m, 3H), 2.87 (d, $J=3.9$ Hz, 1H), 2.89-3.10 (m, 3H), 4.08-4.21 (m, 2H), 4.49 (dd, $J=8.7$, $5.1 \mathrm{~Hz}, 1 \mathrm{H}), 7.66$ (d, $J=8.6 \mathrm{~Hz}, 1 \mathrm{H}) ;{ }^{13} \mathrm{C} \mathrm{NMR}\left(\mathrm{CDCl}_{3}, 75.5 \mathrm{MHz}\right) \delta 17.6,18.0,18.9,18.9,19.9$ (t, ${ }^{3} J_{\mathrm{CF}}=4.0 \mathrm{~Hz}$ ), 27.7, 27.9, 30.7, 31.5, 46.9, 50.9 (q, $\left.{ }^{2} J_{\mathrm{CF}}=25.3 \mathrm{~Hz}\right), 57.4,63.6,68.2,81.5$, $124.4\left(\mathrm{q},{ }^{1} J_{\mathrm{CF}}=280.5 \mathrm{~Hz}\right.$ ), 166.4 (q, ${ }^{3} J_{\mathrm{CF}}=2.3 \mathrm{~Hz}$ ), 171.7, 174.6, (the signals from the $\mathrm{C}_{8} \mathrm{~F}_{17}$ group were obscured due to their low intensity); ${ }^{19} \mathrm{~F} \mathrm{NMR}\left(\mathrm{CDCl}_{3}, 282.4 \mathrm{MHz}\right) \delta-67.3$ (d, $J_{\mathrm{HF}}=$ $7.7 \mathrm{~Hz}, 3 \mathrm{~F}$ ), -81.5 (t, ${ }^{3} \mathrm{JFF}_{\mathrm{FF}}=10.3 \mathrm{~Hz}, 3 \mathrm{~F}$ ), -114.9 (br, 2F), -122.3 (br, 6F), -123.3 (br, 2F), -124.0 (br, 2F), -126.7 (br, 2F). NMR data for the minor diastereoisomer: ${ }^{19} \mathrm{~F}$ NMR $\left(\mathrm{CDCl}_{3}, 282.4\right.$ MHz) $\delta$-66.2 (d, $\left.J_{\mathrm{HF}}=9.5 \mathrm{~Hz}, 3 \mathrm{~F}\right)$. HRMS (FAB): Calcd for $\mathrm{C}_{29} \mathrm{H}_{37} \mathrm{~F}_{20} \mathrm{~N}_{2} \mathrm{O}_{5}\left(\mathrm{M}^{+}+1\right)$ : 873.2383; found: 873.2370 .

\section{Synthesis of dimethyl-(3-(perfluorooctyl)propyl)vinylsilane, 9.}

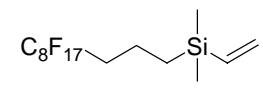

A solution of 3-(perfluorooctyl)propyl iodide 8 (700 mg, $1.2 \mathrm{mmol})$ in $\mathrm{Et}_{2} \mathrm{O}(25 \mathrm{~mL})$ was added to a flask containing a stirred solution of $t$-BuLi $(1.7 \mathrm{M}$ in pentane, $1.76 \mathrm{~mL}, 3.0 \mathrm{mmol})$ at -78 ${ }^{\circ} \mathrm{C}$, and the mixture was warmed up to $-10{ }^{\circ} \mathrm{C}$. After stirring for $30 \mathrm{~min}$, the reaction was again cooled to $-78{ }^{\circ} \mathrm{C}$ and a solution of chloro(dimethyl)vinylsilane $(0.25 \mathrm{~mL}, 1.8 \mathrm{mmol})$ in $\mathrm{Et}_{2} \mathrm{O}$ (4 $\mathrm{mL}$ ) was added. After stirring at room temperature for $5 \mathrm{~h}$, the reaction was quenched with water and the phases were separated. The organic layer was washed with brine, dried over $\mathrm{Na}_{2} \mathrm{SO}_{4}$, and concentrated at reduced pressure. The crude material was purified by means of flash column 
chromatography with hexane to give $510 \mathrm{mg}$ of $\mathbf{9}$ as a colorless oil (78\% yield). $R_{f}=0.84$ (Hexane). ${ }^{1} \mathrm{H}$ NMR ( $\left.\mathrm{CDCl}_{3}, 300 \mathrm{MHz}\right) \delta 0.09$ (br, 6H), 0.64-0.67 (m, 2H), 1.57-1.69 (m, 2H), 1.99-2.17 (m, 2H), 5.66-5.74 (m, 1H), 5.95-6.19 (m, 2H); $\left.{ }^{19} \mathrm{~F} \mathrm{NMR} \mathrm{(CDCl} 3,282.4 \mathrm{MHz}\right) \delta-81.0$ (t, $\left.{ }^{3} J_{\mathrm{FF}}=10.0 \mathrm{~Hz}, 3 \mathrm{~F}\right),-114.6$ (br, 2F), -122.0 (br, 6F), -122.8 (br, 2F), -123.7 (br, 2F), 126.3 (br, 2F).

\section{Synthesis of Fmoc-Val- ${ }^{\mathrm{F}}$ TMSE, 12a.}

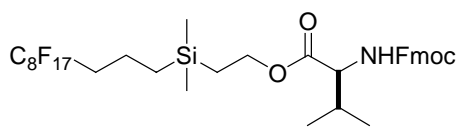

Following General Procedure C, from 11 (400 mg, $0.71 \mathrm{mmol}$ ) and Fmoc-L-valine (240 mg,

$0.71 \mathrm{mmol}$ ), $503 \mathrm{mg}$ of 12a were obtained as a colorless oil (80\% yield). $[\alpha]_{\mathrm{D}}{ }^{25}-2.4$ (c 0.9 , $\left.\mathrm{CHCl}_{3}\right) .{ }^{1} \mathrm{H}$ NMR $\left(\mathrm{CDCl}_{3}, 300 \mathrm{MHz}\right) \delta 0.07$ (s, 6H), 0.59-0.65 (m, 2H), $0.92(\mathrm{~d}, J=7.2 \mathrm{~Hz}, 3 \mathrm{H})$, 0.98 (d, J= 6.8 Hz, 3H), 1.02-1.08 (m, 2H), 1.58-1.67 (m, 2H), 2.00-2.21 (m, 3H), 4.20-4.30 (m, 4H), 4.38-4.41 (m, 2H), 5.22 (d, $J=9.0 \mathrm{~Hz}, 1 \mathrm{H}), 7.32$ (t, $J=7.1 \mathrm{~Hz}, 2 \mathrm{H}), 7.40$ (t, $J=7.5 \mathrm{~Hz}, 2 \mathrm{H})$, 7.61 (d, $J=7.5 \mathrm{~Hz}, 2 \mathrm{H}), 7.77$ (d, $J=7.5 \mathrm{~Hz}, 2 \mathrm{H}) ;{ }^{13} \mathrm{C} \mathrm{NMR}\left(\mathrm{CDCl}_{3}, 75.5 \mathrm{MHz}\right) \delta-3.1,15.1$, 16.3, 17.9, 19.3, 30.4, 31.7, $34.8\left(\mathrm{t},{ }^{2} J_{\mathrm{CF}}=21.9 \mathrm{~Hz}\right), 47.6,59.4,63.6,67.4,120.3,125.4,127.4$ 128.0, 141.7, 144.2, 156.6, 172.6 (the signals from the $\mathrm{C}_{8} \mathrm{~F}_{17}$ group were obscured due to their low intensity); ${ }^{19} \mathrm{~F}$ NMR $\left(\mathrm{CDCl}_{3}, 282.4 \mathrm{MHz}\right) \delta-81.4\left(\mathrm{t},{ }^{3} J_{\mathrm{FF}}=9.9 \mathrm{~Hz}, 3 \mathrm{~F}\right),-115.0(\mathrm{br}, 2 \mathrm{~F}),-$ 122.6 (br, 6F), -123.4 (br, 2F), -124.2 (br, 2F), -126.7 (br, 2F).

\section{Synthesis of Fmoc-Ala- ${ }^{\mathrm{F}}$ TMSE, $12 \mathrm{~b}$.}

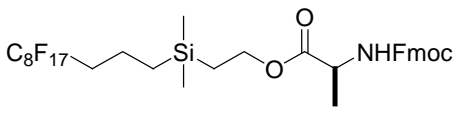

Following General Procedure C, from 11 (530 mg, $0.94 \mathrm{mmol}$ ) and Fmoc-L-alanine (293 mg, $0.94 \mathrm{mmol}$ ), $480 \mathrm{mg}$ of $\mathbf{1 2 b}$ were obtained as a colorless oil (60\% yield). [ $\alpha]_{\mathrm{D}}{ }^{25}-2.0$ (c 0.9 , $\left.\mathrm{CHCl}_{3}\right) .{ }^{1} \mathrm{H}$ NMR $\left(\mathrm{CDCl}_{3}, 300 \mathrm{MHz}\right) \delta 0.07$ (s, 6H), 0.60-0.65 (m, 2H), 1.02-1.08 (m, 2H), 1.43 (d, $J=7.0 \mathrm{~Hz}, 3 \mathrm{H}$ ), 1.57-1.68 (m, 2H), 2.01-2.18 (m, 2H), 4.21-4.27 (m, 3H), 4.35-4.41 (m, 3H), 5.37 (d, $J=7.2 \mathrm{~Hz}, 1 \mathrm{H}), 7.29-7.43(\mathrm{~m}, 4 \mathrm{H}), 7.59-7.78(\mathrm{~m}, 4 \mathrm{H}) ;{ }^{13} \mathrm{C} \mathrm{NMR}\left(\mathrm{CDCl}_{3}, 75.5 \mathrm{MHz}\right) \delta$ -3.3, 15.0, 15.4, 16.0, 18.9, 34.7 (t, ${ }^{2} J_{\mathrm{CF}}=22.2 \mathrm{~Hz}$ ), 47.4, 50.0, 63.7, 67.2, 120.2, 125.3, 127.3 $127.9,141.5,144.0,144.2,155.9,173.4$ (the signals from the $\mathrm{C}_{8} \mathrm{~F}_{17}$ group were obscured due to 
their low intensity); ${ }^{19} \mathrm{~F}$ NMR $\left(\mathrm{CDCl}_{3}, 282.4 \mathrm{MHz}\right) \delta-81.3\left(\mathrm{t},{ }^{3} J_{\mathrm{FF}}=9.9 \mathrm{~Hz}, 3 \mathrm{~F}\right),-114.9$ (br, $\left.2 \mathrm{~F}\right)$, -122.4 (br, 6F), -123.2 (br, 2F), -124.1 (br, 2F), -126.6 (br, 2F).

\section{Synthesis of valine benzyl ester, 13.}<smiles></smiles>

Following General Procedure A, from 12a (17 mg, $0.019 \mathrm{mmol}$ ), $2.5 \mathrm{mg}$ of 13 were obtained as a colorless oil (62\% yield). The spectroscopic data and optical rotation measurements of this compound matched those of a commercial sample.

\section{Synthesis of H-Val- ${ }^{\mathrm{F}}$ TMSE, $14 a$.}

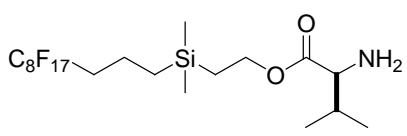

Following General Procedure D, from 12a (35 mg, $0.040 \mathrm{mmol}$ ), $25 \mathrm{mg}$ of 14a were obtained as a colorless oil (95\% yield). $[\alpha]_{\mathrm{D}}{ }^{25}-12.2\left(c\right.$ 0.4, $\left.\mathrm{CHCl}_{3}\right) .{ }^{1} \mathrm{H} \mathrm{NMR}\left(\mathrm{CDCl}_{3}, 300 \mathrm{MHz}\right) \delta 0.06$ (s, 6H), 0.60-0.65 (m, 2H), 0.90 (d, $J=6.8 \mathrm{~Hz}, 3 \mathrm{H}), 0.97$ (d, $J=6.8 \mathrm{~Hz}, 3 \mathrm{H}), 0.98-1.05$ (m, 2H),

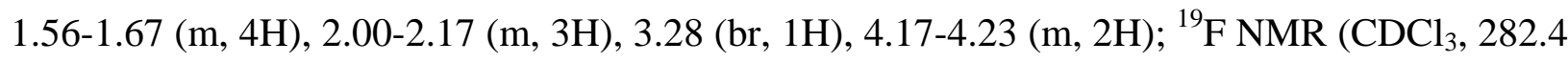
$\mathrm{MHz}) \delta-81.2\left(\mathrm{t},{ }^{3} J_{\mathrm{FF}}=8.4 \mathrm{~Hz}, 3 \mathrm{~F}\right),-114,8$ (br, 2F), -122.4 (br, 6F), -123.2 (br, 2F), -124.1 (br, 2F), -126.6 (br, 2F).

\section{Synthesis of H-Ala- ${ }^{\mathrm{F}}$ TMSE, $14 \mathrm{~b}$.}

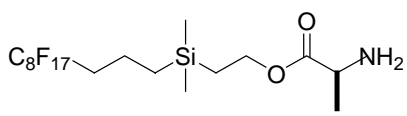

Following General Procedure D, from 12b (300 mg, $0.35 \mathrm{mmol}$ ), $185 \mathrm{mg}$ of 14b were obtained as a colorless oil (85\% yield). $[\alpha]_{\mathrm{D}}{ }^{25}+2.1$ (c 1.05, $\left.\mathrm{CHCl}_{3}\right) .{ }^{1} \mathrm{H} \mathrm{NMR}\left(\mathrm{CDCl}_{3}, 300 \mathrm{MHz}\right) \delta 0.04$ (s, 6H), 0.57-0.63 (m, 2H), 0.98-1.04 (m, 2H), 1.30 (d, J= 7.5 Hz, 3H), 1.56-1.62 (m, 4H), 1.97-

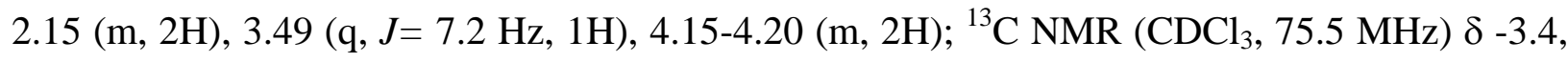
14.9, 15.3, 15.9, 20.7, $34.5\left(\mathrm{t},{ }^{2} J_{\mathrm{CF}}=22.2 \mathrm{~Hz}\right.$ ), 50.2, 62.8, 176.8 (the signals from the $\mathrm{C}_{8} \mathrm{~F}_{17}$ group were obscured due to their low intensity); ${ }^{19} \mathrm{~F} \mathrm{NMR}\left(\mathrm{CDCl}_{3}, 282.4 \mathrm{MHz}\right) \delta-81.3\left(\mathrm{t},{ }^{3} J_{\mathrm{FF}}=8.4\right.$ Hz, 3F), -114,9 (br, 2F), -122.4 (br, 6F), -123.2 (br, 2F), -124.1 (br, 2F), -126.6 (br, 2F). 
Synthesis of Boc-Ala-Val- ${ }^{\mathrm{F}}$ TMSE, $15 a$.

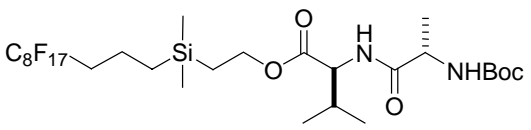

Following General Procedure G, from 14 (45 mg, 0.067 mmol) and Boc-L-alanine (15 mg, 0.081 mmol), $46 \mathrm{mg}$ of $\mathbf{1 5 a}$ were obtained as a colorless oil (81\% yield). [ $\alpha]_{\mathrm{D}}{ }^{25}-14.2$ (c 1.05, $\mathrm{CHCl}_{3}$ ). ${ }^{1} \mathrm{H}$ NMR $\left(\mathrm{CDCl}_{3}, 300 \mathrm{MHz}\right) \delta 0.06$ (s, 6H), 0.59-0.65 (m, 2H), 0.90 (d, J=6.9 Hz, 3H), 0.93 (d, $J=6.9 \mathrm{~Hz}, 3 \mathrm{H}), 1.01-1.06$ (m, 2H), 1.35 (d, $J=7.2 \mathrm{~Hz}, 3 \mathrm{H}), 1.44$ (s, 9H), 1.58-1.66 (m, 2H), 2.00-2.23 (m, 3H), 4.19-4.24 (m, 3H), 4.49 (dd, $J=9.0,4.8 \mathrm{~Hz}, 1 \mathrm{H}), 4.96$ (br, 1H), 6.62 (d, $J=$ 8.7 Hz, 1H); ${ }^{13} \mathrm{C} \mathrm{NMR}\left(\mathrm{CDCl}_{3}, 75.5 \mathrm{MHz}\right) \delta-2.9,15.4\left(\mathrm{t},{ }^{3} J_{\mathrm{CF}}=4.5 \mathrm{~Hz}\right), 15.7,16.5,18.1,19.6$, 28.9, 30.3, 31.9, 35.0 (t, ${ }^{2} J_{\mathrm{CF}}=21.9 \mathrm{~Hz}$ ), 50.6, 57.7, 63.8, 80.8, 156.1, 172.4, 173.0, (the signals from the $\mathrm{C}_{8} \mathrm{~F}_{17}$ group were obscured due to their low intensity); ${ }^{19} \mathrm{~F}$ NMR ( $\left.\mathrm{CDCl}_{3}, 282.4 \mathrm{MHz}\right) \delta$ $-80.7\left(\mathrm{t},{ }^{3} J_{\mathrm{FF}}=8.4 \mathrm{~Hz}, 3 \mathrm{~F}\right),-114.4$ (br, 2F), -121.8 (br, 6F), -122.7 (br, 2F), -123.6 (br, 2F), 126.1 (br, 2F).

\section{Synthesis of Boc-Phg-Val- ${ }^{\mathrm{F}}$ TMSE, $15 \mathrm{~b}$.}

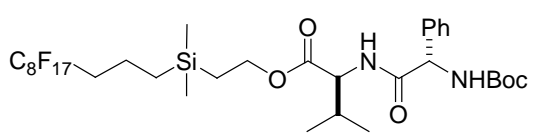

Following General Procedure G, from 14a (35 mg, $0.053 \mathrm{mmol}$ ) and Boc-L-phenylglycine (16 $\mathrm{mg}, 0.063 \mathrm{mmol}), 30 \mathrm{mg}$ of $\mathbf{1 5 b}$ were obtained as a colorless oil (64\% yield). $[\alpha]_{\mathrm{D}}{ }^{25}+19.1$ (c 0.9, $\left.\mathrm{CHCl}_{3}\right) .{ }^{1} \mathrm{H} \mathrm{NMR}\left(\mathrm{CDCl}_{3}, 300 \mathrm{MHz}\right) \delta 0.01$ (s, 6H), 0.54-0.60 (m, 2H), 0.87 (d, J= 6.9 Hz, 3H), 0.90-0.95 (m, 2H), 0.93 (d, J=6.8 Hz, 3H), 1.38 (s, 9H), 1.52-1.63 (m, 2H), 1.97-2.20 (m, 3H), 4.07-4.13 (m, 2H), 4.43 (dd, $J=8.6,4.8 \mathrm{~Hz}, 1 \mathrm{H}), 5.15$ (br, 1H), 5.69 (br, 1H), 6.19 (d, $J=$ 8.7 Hz, 1H), 7.27-7.36 (m, 5H); ${ }^{13} \mathrm{C}$ NMR $\left(\mathrm{CDCl}_{3}, 75.5 \mathrm{MHz}\right) \delta-3.5,14.8,15.1,15.8,17.7$, 18.9, 28.2, 31.4, $34.4\left(\mathrm{t},{ }^{2} J_{\mathrm{CF}}=21.9 \mathrm{~Hz}\right), 57.4$, 58.5, 63.2, 80.2, 127.2, 128.4, 129.0, 137.9, 155.1, 170.2, 171.2, (the signals from the $\mathrm{C}_{8} \mathrm{~F}_{17}$ group were obscured due to their low intensity); ${ }^{19} \mathrm{~F}$ NMR $\left(\mathrm{CDCl}_{3}, 282.4 \mathrm{MHz}\right) \delta-81.5\left(\mathrm{t},{ }^{3} J_{\mathrm{FF}}=8.4 \mathrm{~Hz}, 3 \mathrm{~F}\right),-115.1$ (br, 2F), -122.5 (br, 6F), -123.4 (br, 2F), -124.3 (br, 2F), -126.8 (br, 2F). 
Synthesis of Boc-(S)- $\beta^{2}$-HomoAla-Val- ${ }^{\mathrm{F}}$ TMSE, 15c.

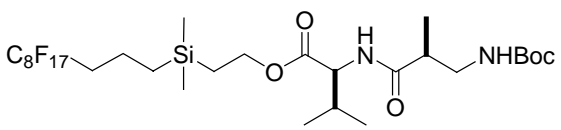

Following General Procedure G, from 14a (45 mg, $0.067 \mathrm{mmol}$ ) and (S)-Boc- $\beta^{2}$-HomoAla-OH (18 mg, $0.088 \mathrm{mmol}), 49 \mathrm{mg}$ of $\mathbf{1 5 c}$ were obtained as a colorless oil (85\% yield). $[\alpha]_{\mathrm{D}}{ }^{25}+18.3$ (c 1.3, $\left.\mathrm{CHCl}_{3}\right) .{ }^{1} \mathrm{H} \mathrm{NMR}\left(\mathrm{CDCl}_{3}, 300 \mathrm{MHz}\right) \delta 0.04$ (br, 6H), 0.55-0.61 (m, 2H), 0.86 (d, J= 6.8 Hz, 3H), 0.90 (d, $J=6.8 \mathrm{~Hz}, 3 \mathrm{H}), 0.97-1.03$ (m, 2H), 1.12 (d, $J=6.5 \mathrm{~Hz}, 3 \mathrm{H}), 1.39$ (s, 9H), 1.51-1.63 (m, 2H), 1.95-2.19 (m, 3H), 2.51-2.53 (m, 1H), 3.08-3.17 (m, 1H), 3.23-3.30 (m, 1H), 4.11-4.24 (m, 2H), 4.44 (dd, $J=8.8,4.4 \mathrm{~Hz}, 1 \mathrm{H}), 5.18$ (br, $1 \mathrm{H}), 5.92$ (d, $J=8.7 \mathrm{~Hz}, 1 \mathrm{H}) ;{ }^{13} \mathrm{C} \mathrm{NMR}\left(\mathrm{CDCl}_{3}\right.$, $75.5 \mathrm{MHz}) \delta-3.1,15.2,15.5,15.8,16.3,18.0,19.4,28.7,31.3,34.8\left(\mathrm{t},{ }^{2} J_{\mathrm{CF}}=21.9 \mathrm{~Hz}\right), 41.6$, 44.0, 57.4, 63.7, 79.6, 156.5, 172.5, 175.5 (the signals from the $\mathrm{C}_{8} \mathrm{~F}_{17}$ group were obscured due to their low intensity); ${ }^{19} \mathrm{~F}$ NMR $\left(\mathrm{CDCl}_{3}, 282.4 \mathrm{MHz}\right) \delta-80.8\left(\mathrm{t},{ }^{3} J_{\mathrm{FF}}=9.1 \mathrm{~Hz}, 3 \mathrm{~F}\right),-114.3$ (br, 2F), -121.9 (br, 6F), -122.7 (br, 2F), -123.6 (br, 2F), -126.1 (br, 2F).

\section{Synthesis of Boc-Ile-Ala- ${ }^{\mathrm{F}}$ TMSE, 15d.}

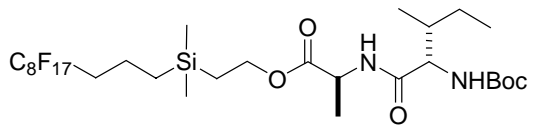

Following General Procedure G, from 14b (41 mg, $0.065 \mathrm{mmol}$ ) and Boc-L-isoleucine (18 mg, $0.078 \mathrm{mmol}), 48 \mathrm{mg}$ of $\mathbf{1 5 d}$ were obtained as a colorless oil (87\% yield). $[\alpha]_{\mathrm{D}}{ }^{25}-9.2$ (c 0.73 , $\left.\mathrm{CHCl}_{3}\right) .{ }^{1} \mathrm{H} \mathrm{NMR}\left(\mathrm{CDCl}_{3}, 300 \mathrm{MHz}\right) \delta 0.00$ (s, 6H), 0.52-0.59 (m, 2H), 0.82-0.94 (m, 6H), 0.961.00 (m, 2H), 1.04-1.22 (m, 2H), 1.34 (d, $J=6.9$ Hz, 3H), 1.38 (s, 9H), 1.49-1.61 (m, 2H), 1.761.82 (m, 1H), 1.94-2.11 (m, 2H), 3.88-3.93 (m, 1H), 4.13-4.18 (m, 2H), 4.43-4.52 (m, 1H), 5.02 (d, $J=8.1 \mathrm{~Hz}, 1 \mathrm{H}), 6.42$ (d, $J=7.2 \mathrm{~Hz}, 1 \mathrm{H}) ;{ }^{13} \mathrm{C}$ NMR $\left(\mathrm{CDCl}_{3}, 75.5 \mathrm{MHz}\right) \delta-3.2,11.6,15.0$, 15.4, 15.7, 16.0, 18.6, 25.0, 28.5, $34.7\left(\mathrm{t},{ }^{3} J_{\mathrm{CF}}=22.2 \mathrm{~Hz}\right), 37.6,48.4$, 59.4, 63.7, 80.1, 156.0, 171.3, 173.0, (the signals from the $\mathrm{C}_{8} \mathrm{~F}_{17}$ group were obscured due to their low intensity); ${ }^{19} \mathrm{~F}$

NMR $\left(\mathrm{CDCl}_{3}, 282.4 \mathrm{MHz}\right) \delta-81.3\left(\mathrm{t},{ }^{3} J_{\mathrm{FF}}=9.8 \mathrm{~Hz}, 3 \mathrm{~F}\right),-114.9$ (br, 2F), -122.4 (br, 6F), -123.2 (br, 2F), -124.1 (br, 2F), -126.6 (br, 2F). 


\section{Synthesis of Boc-Phe-Ala- ${ }^{\mathrm{F}}$ TMSE, $15 \mathrm{e}$.}

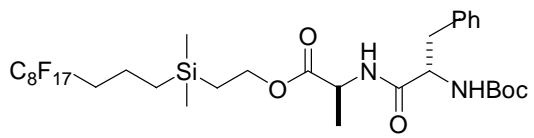

Following General Procedure G, from 14b (73 mg, $0.115 \mathrm{mmol}$ ) and Boc-L-phenylalanine (37 mg, $0.138 \mathrm{mmol}), 83 \mathrm{mg}$ of $\mathbf{1 5 e}$ were obtained as a white solid (80\% yield). mp: $44-45^{\circ} \mathrm{C}$. $[\alpha]_{\mathrm{D}}{ }^{25}+9.5\left(c \mathrm{0.7}, \mathrm{CHCl}_{3}\right) .{ }^{1} \mathrm{H} \mathrm{NMR}\left(\mathrm{CDCl}_{3}, 300 \mathrm{MHz}\right) \delta 0.00(\mathrm{~s}, 6 \mathrm{H}), 0.53-0.59(\mathrm{~m}, 2 \mathrm{H}), 0.93-$ 0.98 (m, 2H), 1.28 (d, J= 7.1 Hz, 3H), 1.34 (s, 9H), 1.50-1.61 (m, 2H), 1.94-2.12 (m, 2H), 3.01 (d, $J=6.6 \mathrm{~Hz}, 2 \mathrm{H}), 4.06-4.16$ (m, 2H), 4.17-4.46 (m, 2H), 4.93 (br, 1H), 6.41 (d, J= 7.2 Hz, 1H), 7.13-7.22 (m, 5H); ${ }^{13} \mathrm{C} \mathrm{NMR}\left(\mathrm{CDCl}_{3}, 75.5 \mathrm{MHz}\right) \delta$-3.1, 15.1, 15.5 16.1, 18.7, 28.6, 34.8 (t,

${ }^{2} J_{\mathrm{CF}}=21.9 \mathrm{~Hz}$ ), 38.7, 48.6, 56.0, 63.8, 80.6, 127.3, 129.0, 129.7, 136.9, 155.7, 171.1, 172.9 (the signals from the $\mathrm{C}_{8} \mathrm{~F}_{17}$ group were obscured due to their low intensity); ${ }^{19} \mathrm{~F}$ NMR ( $\mathrm{CDCl}_{3}, 282.4$ $\mathrm{MHz}) \delta-81.5\left(\mathrm{t},{ }^{3} J_{\mathrm{FF}}=9.8 \mathrm{~Hz}, 3 \mathrm{~F}\right),-115.1$ (br, 2F), -122.6 (br, 6F), -123.4 (br, 2F), -124.3 (br, 2F), -126.8 (br, 2F).

\section{Synthesis of Boc-Ala-Val-OBn, 16a.}

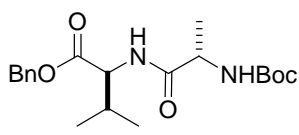

Following General Procedure A, from 15a (16 mg, $0.018 \mathrm{mmol}), 6.6 \mathrm{mg}$ of 16a were obtained as a colorless oil (91\% yield). $[\alpha]_{\mathrm{D}}{ }^{25}-36.6$ (c 0.67, $\left.\mathrm{CHCl}_{3}\right) .{ }^{1} \mathrm{H} \mathrm{NMR}\left(\mathrm{CDCl}_{3}, 300 \mathrm{MHz}\right) \delta 0.80$ (d, $J=6.9 \mathrm{~Hz}, 3 \mathrm{H}$ ), 0.85 (d, $J=6.9 \mathrm{~Hz}, 3 \mathrm{H}), 1.29$ (d, $J=7.1 \mathrm{~Hz}, 3 \mathrm{H}), 1.38$ (s, 9H), 2.08-2.19 (m, 1H), 4.03-4.12 (m, 1H), 4.52 (dd, $J=8.9,4.8 \mathrm{~Hz}, 1 \mathrm{H}), 4.92$ (d, $J=7.0 \mathrm{~Hz}, 1 \mathrm{H}), 5.07$ (d, $J=12.3 \mathrm{~Hz}$, 1H), 5.14 (d, $J=12.2 \mathrm{~Hz}, 1 \mathrm{H}), 6.58$ (br, $1 \mathrm{H}), 7.24-7.33(\mathrm{~m}, 5 \mathrm{H}) ;{ }^{13} \mathrm{C} \mathrm{NMR}\left(\mathrm{CDCl}_{3}, 75.5 \mathrm{MHz}\right) \delta$ 17.4, 17.7, 18.9, 28.2, 31.1, 49.0, 56.9, 66.9, 79.9, 128.2, 128.3, 128.5, 135.3, 155.5, 171.5, 172.6. HRMS (FAB): Calcd for $\mathrm{C}_{20} \mathrm{H}_{31} \mathrm{~N}_{2} \mathrm{O}_{5}\left(\mathrm{M}^{+}+1\right)$ : 379.2233; found: 379.2242.

\section{Synthesis of Boc-Phg-Val-OBn, $16 b$.}<smiles>CC(C)C(NC(=O)[C@H](NC(=O)O)c1ccccc1)C(=O)O</smiles>

Following General Procedure A, from 15b (13 mg, $0.014 \mathrm{mmol}), 5 \mathrm{mg}$ of $\mathbf{1 6 b}$ were obtained as a white solid (79\% yield). mp: $107-108{ }^{\circ} \mathrm{C} .[\alpha]_{\mathrm{D}}{ }^{25}+26.8$ (c $\left.0.94, \mathrm{CHCl}_{3}\right) .{ }^{1} \mathrm{H} \mathrm{NMR}\left(\mathrm{CDCl}_{3}, 300\right.$ 
MHz) $\delta 0.77$ (d, $J=6.9 \mathrm{~Hz}, 3 \mathrm{H}$ ), 0.84 (d, $J=6.9 \mathrm{~Hz}, 3 \mathrm{H}), 1.35$ (s, 9H), 2.06-2.16 (m, 1H), 4.49 (dd, $J=8.6,4.7 \mathrm{~Hz}, 1 \mathrm{H}$ ), 4.98 (d, $J=12.2 \mathrm{~Hz}, 1 \mathrm{H}$ ), 5.05 (d, $J=12.2 \mathrm{~Hz}, 1 \mathrm{H}$ ), 5.12 (br, 1H), 5.64 (br, $1 \mathrm{H}), 6.17$ (d, $J=8.5 \mathrm{~Hz}, 1 \mathrm{H}), 7.18-7.31(\mathrm{~m}, 10 \mathrm{H}) ;{ }^{13} \mathrm{C} \mathrm{NMR}\left(\mathrm{CDCl}_{3}, 75.5 \mathrm{MHz}\right) \delta 17.5$, 18.8, 28.2, 31.3, 57.3, 58.5, 66.9, 80.0, 127.1, 128.2, 128.2, 128.3, 128.5, 128.8, 135.1, 137.7, 155.1, 170.2, 171.0. HRMS (FAB): Calcd for $\mathrm{C}_{25} \mathrm{H}_{33} \mathrm{~N}_{2} \mathrm{O}_{5}\left(\mathrm{M}^{+}+1\right)$ : 441.2389; found: 441.2406.

\section{Synthesis of Boc-(S)- $\beta^{2}$-HomoAla-Val-OBn, 16c.}<smiles>CC(C)C(C)C(=O)NC(C(C)C)C(C)C</smiles>

Following General Procedure A, from 15c (14 mg, $0.016 \mathrm{mmol}), 6 \mathrm{mg}$ of 16c were obtained as a white solid (92\% yield). mp: $105-107{ }^{\circ} \mathrm{C} .[\alpha]_{\mathrm{D}}{ }^{25}+3.8$ (c 1.0, $\left.\mathrm{CHCl}_{3}\right) .{ }^{1} \mathrm{H}$ NMR $\left(\mathrm{CDCl}_{3}, 300\right.$ MHz) $\delta 0.79$ (d, $J=6.9 \mathrm{~Hz}, 3 \mathrm{H}$ ), 0.85 (d, $J=6.9 \mathrm{~Hz}, 3 \mathrm{H}), 1.09$ (d, $J=7.2 \mathrm{~Hz}, 3 \mathrm{H}), 1.36$ (s, 9H), 2.07-2.18 (m, 1H), 2.50-2.51 (m, 1H) 3.04-3.13 (m, 1H), 3.20-3.28 (m, 1H), 4.50 (dd, $J=8.7,4.5$ Hz, 1H), 5.07 (d, $J=12.0 \mathrm{~Hz}, 1 \mathrm{H}$ ), 5.10-5.20 (br, 1H), 5.15 (d, $J=12.3 \mathrm{~Hz}, 1 \mathrm{H}$ ), 5.95 (d, $J=8.7$ $\mathrm{Hz}, 1 \mathrm{H}), 7.25-7.35$ (m, 5H); ${ }^{13} \mathrm{C}$ NMR $\left(\mathrm{CDCl}_{3}, 75.5 \mathrm{MHz}\right) \delta 15.4,17.6,19.0,28.4,31.0,41.3$, 43.7, 57.0, 67.1, 79.3, 128.4, 128.5, 128.6, 135.3, 156.1, 171.9, 175.2. HRMS (FAB): Calcd for $\mathrm{C}_{21} \mathrm{H}_{33} \mathrm{~N}_{2} \mathrm{O}_{5}\left(\mathrm{M}^{+}+1\right)$ : 393.2389; found: 393.2401 .

\section{Synthesis of Boc-Ile-Ala-OBn, 16d.}

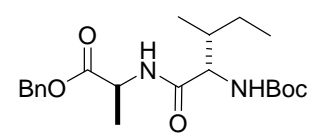

Following General Procedure A, from 15d (20 $\mathrm{mg}, 0.023 \mathrm{mmol}$ ), $8 \mathrm{mg}$ of $\mathbf{1 6 d}$ were obtained as a white solid (85\% yield). mp: 108-109 ${ }^{\circ} \mathrm{C} .[\alpha]_{\mathrm{D}}{ }^{25}-23.5$ (c 0.9, $\left.\mathrm{CHCl}_{3}\right) .{ }^{1} \mathrm{H}$ NMR $\left(\mathrm{CDCl}_{3}, 300\right.$ MHz) $\delta$ 0.80-0.86 (m, 6H), 0.99-1.22 (m, 2H), 1.26-1.37 (m, 12H), 1.76 (br, 1H), 3.90 (t, $J=7.5$ $\mathrm{Hz}, 1 \mathrm{H}), 4.52-4.61$ (m, 1H), 5.02-5.15 (m, 3H), 6.46 (d, $J=6.9 \mathrm{~Hz}, 1 \mathrm{H}), 7.24-7.32$ (m, 5H); ${ }^{13} \mathrm{C}$ NMR $\left(\mathrm{CDCl}_{3}, 75.5 \mathrm{MHz}\right) \delta 11.5,15.4,18.3,24.9,28.5,37.6,48.3,59.2,67.2,79.9,128.3$, 128.5, 128.7, 135.5, 156.0, 171.5, 172.7. HRMS (FAB): Calcd for $\mathrm{C}_{21} \mathrm{H}_{33} \mathrm{~N}_{2} \mathrm{O}_{5}\left(\mathrm{M}^{+}+1\right)$ : 393.2389; found: 339.2374 . 


\section{Synthesis of Boc-Phg-Ala-Val- ${ }^{\mathrm{F}}$ TMSE, 17.}

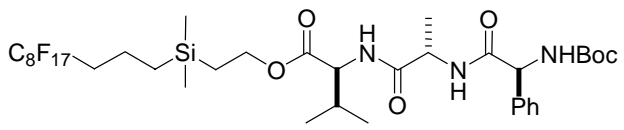

TFA (70 $\mu \mathrm{l}, 0.91 \mathrm{mmol})$ was added to a solution of 15a (38 $\mathrm{mg}, 0.042 \mathrm{mmol})$ in $\mathrm{CH}_{2} \mathrm{Cl}_{2}(0.25$ $\mathrm{mL}$ ). The mixture was stirred at room temperature for $16 \mathrm{~h}$, and then concentrated at reduced pressure. The residue was reacted following General Procedure G, with Boc-L-phenylglycine (14 mg, $0.054 \mathrm{mmol}$ ), to give $26 \mathrm{mg}$ of 17 as a white solid (63\% yield). mp: $84-85{ }^{\circ} \mathrm{C}$. [ $\left.\alpha\right]_{\mathrm{D}}{ }^{25}$ +13.1 (c 0.7, $\left.\mathrm{CHCl}_{3}\right) .{ }^{1} \mathrm{H} \mathrm{NMR}\left(\mathrm{CDCl}_{3}, 300 \mathrm{MHz}\right) \delta 0.07$ (br, 6H), 0.58-0.64 (m, 2H), 0.78 (d, $J=6.8 \mathrm{~Hz}, 6 \mathrm{H}), 0.99-1.06$ (m, 2H), 1.36-1.40 (m, 12H), 1.54-1.66 (m, 2H), 1.99-2.13 (m, 3H), 4.17-4.22 (m, 2H), 4.38 (dd, J= 8.9, 4.9 Hz, 1H), 4.46-4.53 (m, 1H), 5.13 (br, 1H), 5.69 (br, 1H),

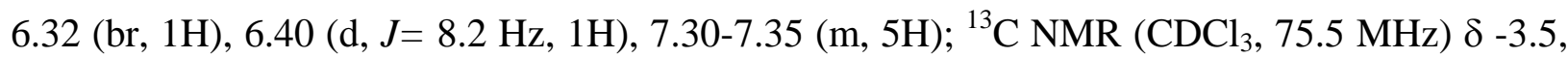
14.1, 14.2, 15.1, 15.9, 17.6, 17.8, 22.6, 28.3, 31.0, 34.4 (t, $\left.{ }^{2} J_{\mathrm{CF}}=22.1 \mathrm{~Hz}\right), 49.1,57.2,63.3,80.3$, 127.1, 128.5, 129.1, 137.6, 155.1, 170.1, 171.3, 171.6, (the signals from the $\mathrm{C}_{8} \mathrm{~F}_{17}$ group were obscured due to their low intensity); ${ }^{19} \mathrm{~F} \mathrm{NMR}\left(\mathrm{CDCl}_{3}, 282.4 \mathrm{MHz}\right) \delta-80.8\left(\mathrm{t},{ }^{3} J_{\mathrm{FF}}=9.1 \mathrm{~Hz}, 3 \mathrm{~F}\right)$, -114.3 (br, 2F), -121.9 (br, 6F), -122.7 (br, 2F), -123.6 (br, 2F), -126.1 (br, 2F).

\section{Synthesis of Boc-Phg-Ala-Val-OBn, 18.}

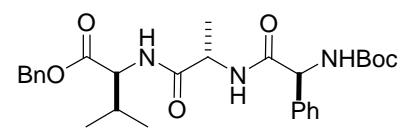

Following General Procedure A, from 17 (13 mg, $0.013 \mathrm{mmol}), 6.5 \mathrm{mg}$ of 18 were obtained as a white solid (95\% yield); mp: $103-104{ }^{\circ} \mathrm{C} .[\alpha]_{\mathrm{D}}{ }^{25}+57.3$ (c 1.03, $\left.\mathrm{CHCl}_{3}\right) .{ }^{1} \mathrm{H}$ NMR $\left(\mathrm{CDCl}_{3}, 300\right.$ MHz) $\delta 0.67$ (d, $J=6.7 \mathrm{~Hz}, 3 \mathrm{H}), 0.69$ (d, $J=6.3 \mathrm{~Hz}, 3 \mathrm{H}), 1.30$ (d, $J=7.0 \mathrm{~Hz}, 3 \mathrm{H}), 1.35$ (s, 9H), 1.98-2.05 (m, 1H), 4.38-4.46 (m, 2H), 5.05 (d, J= $12.2 \mathrm{~Hz}, 1 \mathrm{H}), 5.10$ (br, 1H), 5.14 (d, J= 12.2 Hz, 1H), 5.65 (d, $J=5.9$ Hz, 1H), 6.29 (d, $J=7.4$ Hz, 1H), 6.41 (d, $J=8.8 \mathrm{~Hz}, 1 \mathrm{H}), 7.19-7.33$ (m, $10 \mathrm{H}) ;{ }^{13} \mathrm{C} \mathrm{NMR}\left(\mathrm{CDCl}_{3}, 75.5 \mathrm{MHz}\right) \delta 17.5,18.1,18.7,28.2,31.0,49.0,57.2,58.3,66.9,80.0$, 126.9, 128.2, 128.3, 128.4, 128.5, 128.9, 135.3, 137.8, 155.1, 170.2, 171.4, 171.7. HRMS (FAB): Calcd for $\mathrm{C}_{28} \mathrm{H}_{38} \mathrm{~N}_{3} \mathrm{O}_{6}\left(\mathrm{M}^{+}+1\right)$ : 512.2760; found: 512.2761. 
Synthesis of Michael acceptor- ${ }^{\mathrm{F}}$ TMSE, 19.

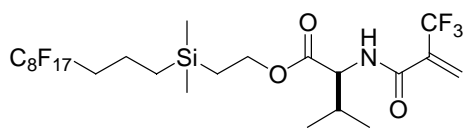

Following General Procedure E, from 14a (63 mg, $0.095 \mathrm{mmol}), 52 \mathrm{mg}$ of 19 were obtained as a colorless oil (70 \% yield). $[\alpha]_{\mathrm{D}}{ }^{25}-4.2$ (c 1.2, $\left.\mathrm{CHCl}_{3}\right) .{ }^{1} \mathrm{H} \mathrm{NMR}\left(\mathrm{CDCl}_{3}, 300 \mathrm{MHz}\right) \delta-0.03$ (s, 6H), 0.53-0.59 (m, 2H), 0.87 (d, J=6.8 Hz, 3H), 0.91 (d, J= 7.2 Hz, 3H), 1.02-1.08 (m, 2H), 1.52-1.60 (m, 2H), 1.96-2.05 (m, 2H), 2.08-2.22 (m, 1H), 4.15-4.21 (m, 2H), 4.56 (dd, J= 4.9, $4.5 \mathrm{~Hz}, 1 \mathrm{H}), 6.20$ (d, $J=1.5 \mathrm{~Hz}, 1 \mathrm{H}), 6.39$ (br, 1H), 6.50 (d, $J=1.5 \mathrm{~Hz}, 1 \mathrm{H}) ;{ }^{13} \mathrm{C}$ NMR $\left(\mathrm{CDCl}_{3}\right.$, $75.5 \mathrm{MHz}) \delta-3.5,15.1,15.9,17.6,18.9,29.7\left(\mathrm{t},{ }^{3} J_{\mathrm{FF}}=3.8 \mathrm{~Hz}\right), 31.4,34.4\left(\mathrm{t},{ }^{2} J_{\mathrm{FF}}=21.9 \mathrm{~Hz}\right)$, 57.5, 63.6, $122.2\left(\mathrm{q},{ }^{1} J_{\mathrm{CF}}=273.1 \mathrm{~Hz}\right), 129.6\left(\mathrm{q},{ }^{3} J_{\mathrm{CF}}=5.2 \mathrm{~Hz}\right), 133.7\left(\mathrm{t},{ }^{2} J_{\mathrm{FF}}=30.8 \mathrm{~Hz}\right), 160.5$, 171.4, (the signals from the $\mathrm{C}_{8} \mathrm{~F}_{17}$ group were obscured due to their low intensity); ${ }^{19} \mathrm{~F}$ NMR $\left(\mathrm{CDCl}_{3}, 282.4 \mathrm{MHz}\right) \delta-64.3(\mathrm{~s}, 3 \mathrm{~F}),-81.3\left(\mathrm{t},{ }^{3} J_{\mathrm{FF}}=10.3 \mathrm{~Hz}, 3 \mathrm{~F}\right),-114.8$ (br, 2F), -122.3 (br, 6F), -123.2 (br, 2F), -123.9 (br, 2F), -126.6 (br, 2F).

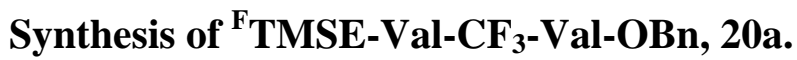

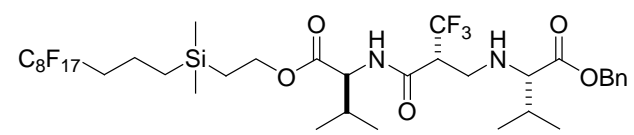

Following General Procedure F, from 19 (25 mg, $0.032 \mathrm{mmol}$ ) and valine benzyl ester hydrochloride (7 mg, $0.032 \mathrm{mmol}$ ), $27.5 \mathrm{mg}$ of 20a were obtained as a white solid (87\% yield) in a 39:1 mixture of diastereoisomers as determined from its ${ }^{19} \mathrm{~F}$ NMR spectrum. NMR data for the major diastereoisomer: ${ }^{1} \mathrm{H}$ NMR $\left(\mathrm{CDCl}_{3}, 300 \mathrm{MHz}\right) \delta-0.01(\mathrm{~s}, 6 \mathrm{H}), 0.52-0.58(\mathrm{~m}, 2 \mathrm{H}), 0.85$ (d, $J=6.8 \mathrm{~Hz}, 3 \mathrm{H}), 0.86$ (d, $J=6.8 \mathrm{~Hz}, 3 \mathrm{H}), 0.91$ (d, $J=6.8 \mathrm{~Hz}, 3 \mathrm{H}), 0.95$ (d, $J=6.7 \mathrm{~Hz}, 3 \mathrm{H}), 0.96-$ 0.99 (m, 2H), 1.19 (br, 1H), 1.49-1.64 (m, 2H), 1.86-2.05 (m, 2H), 2.09-2.17 (m, 2H), 2.92-2.94 (m, 1H), 2.97-3.01 (m, 2H), 3.05 (d, J=5.5 Hz, 1H), 4.11-4.17 (m, 2H), 4.48 (dd, J= 8.7, 4.8 Hz, 1H), 5.09 (d, $J=12.2 \mathrm{~Hz}, 1 \mathrm{H}), 5.13$ (d, $J=12.2 \mathrm{~Hz}, 1 \mathrm{H}), 7.11$ (d, $J=8.7 \mathrm{~Hz}, 1 \mathrm{H}), 7.28$ (br, 5H); ${ }^{13} \mathrm{C} \mathrm{NMR}\left(\mathrm{CDCl}_{3}, 75.5 \mathrm{MHz}\right) \delta-3.5,14.8\left(\mathrm{t},{ }^{3} J_{\mathrm{CF}}=3.8 \mathrm{~Hz}\right), 15.1,15.9,17.6,18.1,19.1,30.9$, 31.6, $34.4\left(\mathrm{t},{ }^{2} J_{\mathrm{CF}}=21.8 \mathrm{~Hz}\right), 45.5\left(\mathrm{t},{ }^{3} J_{\mathrm{CF}}=3.0 \mathrm{~Hz}\right), 51.2\left(\mathrm{t},{ }^{2} J_{\mathrm{CF}}=25.7 \mathrm{~Hz}\right), 57.4,63.2,66.8$, 67.9, $124.4\left(\mathrm{t},{ }^{1} J_{\mathrm{CF}}=280.0 \mathrm{~Hz}\right), 128.4,128.5,128.6,135.5,166.0\left(\mathrm{t},{ }^{3} J_{\mathrm{CF}}=2.9 \mathrm{~Hz}\right), 171.8,175.3$, (the signals from the $\mathrm{C}_{8} \mathrm{~F}_{17}$ group were obscured due to their low intensity); ${ }^{19} \mathrm{~F}$ NMR ( $\mathrm{CDCl}_{3}$, 282.4 MHz) $\delta-66.7\left(\mathrm{~d}, J_{\mathrm{HF}}=8.4 \mathrm{~Hz}, 3 \mathrm{~F}\right),-80.7\left(\mathrm{t},{ }^{3} J_{\mathrm{FF}}=8.4 \mathrm{~Hz}, 3 \mathrm{~F}\right),-114.3$ (br, 2F), -121.9 (br, 
6F), -122.6 (br, 2F), -123.6 (br, 2F), -126.1 (br, 2F). NMR data for the minor diastereoisomer: ${ }^{19} \mathrm{~F}$ NMR $\left(\mathrm{CDCl}_{3}, 282.4 \mathrm{MHz}\right) \delta-66.2\left(\mathrm{~d}, J_{\mathrm{HF}}=9.5 \mathrm{~Hz}, 3 \mathrm{~F}\right)$. HRMS (FAB): Calcd for $\mathrm{C}_{36} \mathrm{H}_{45} \mathrm{~F}_{20} \mathrm{~N}_{2} \mathrm{O}_{5} \mathrm{Si}\left(\mathrm{M}^{+}+1\right)$ : 993.2778; found: 993.2777.

\section{Synthesis of ${ }^{\mathrm{F}}$ TMSE-Val-CF 3 -Leu-OBn, $20 \mathrm{~b}$.}

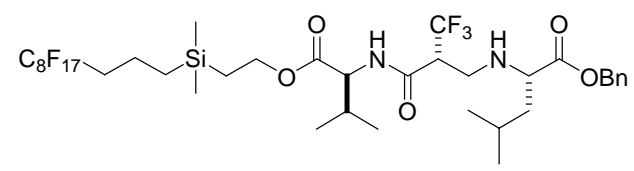

Following General Procedure F, from $19(50 \mathrm{mg}, 0.064 \mathrm{mmol})$ and leucine benzyl ester $p$ toluenesulphonate ( $24 \mathrm{mg}, 0.064 \mathrm{mmol}$ ), $63.5 \mathrm{mg}$ of $\mathbf{2 0 b}$ were obtained as a colorless oil (99\% yield) in a 21:1 mixture of diastereoisomers as determined from its ${ }^{19} \mathrm{~F}$ NMR spectrum. NMR data for the major diastereoisomer: ${ }^{1} \mathrm{H}$ NMR $\left(\mathrm{CDCl}_{3}, 300 \mathrm{MHz}\right) \delta 0.00$ (s, 6H), 0.53-0.59 (m, 2H), 0.88 (d, $J=6.5 \mathrm{~Hz}, 3 \mathrm{H}$ ), 0.90 (d, $J=6.6 \mathrm{~Hz}, 3 \mathrm{H}$ ), 0.93 (d, $J=6.9 \mathrm{~Hz}, 3 \mathrm{H}$ ), 0.98 (d, $J=6.9$ Hz, 3H), 1.03-1.09 (m, 2H), 1.41-1.57 (m, 2H), 1.58-1.75 (m, 3H), 1.80 (br, 1H), 2.02-2.29 (m, 3H), 2.96-3.12 (m, 3H), 3.38 (dd, $J=7.8,6.6 \mathrm{~Hz}, 1 \mathrm{H}$ ), 4.20-4.26 (m, 2H), 4.56 (dd, $J=8.7,4.6$

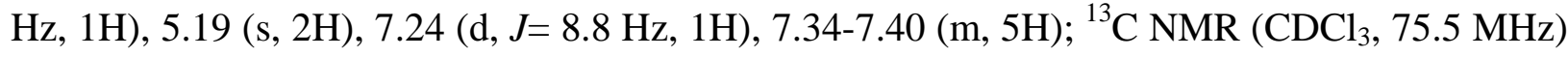
-3.5, 14.8, 15.1, 15.9, 17.5, 19.1, 22.1, 22.5, 24.7, 30.8, 34.4 (t, $\left.{ }^{2} J_{\mathrm{CF}}=22.1 \mathrm{~Hz}\right), 42.4,44.8,50.9$ (q, ${ }^{2} J_{\mathrm{CF}}=25.3 \mathrm{~Hz}$ ), 57.5, 60.6, 63.4, 66.9, 124.3 (q, ${ }^{1} J_{\mathrm{CF}}=280.1 \mathrm{~Hz}$ ), 128.3, 128.4, 128.6, 135.4, $165.9,172.0,175.5$, (the signals from the $\mathrm{C}_{8} \mathrm{~F}_{17}$ group were obscured due to their low intensity);

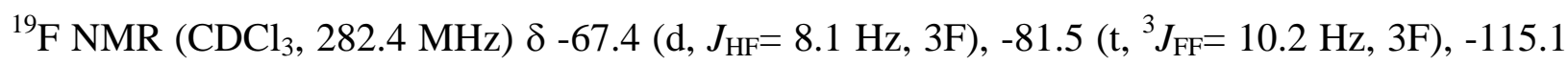
(br, 2F), -122.6 (br, 6H), -123.4 (br, 2F), -124.3 (br, 2F), -126.8 (br, 2F). NMR data for the minor diastereoisomer: ${ }^{19} \mathrm{~F} \mathrm{NMR}\left(\mathrm{CDCl}_{3}, 282.4 \mathrm{MHz}\right) \delta-66.4\left(\mathrm{~d}, J_{\mathrm{HF}}=9.6 \mathrm{~Hz}, 3 \mathrm{~F}\right)$.

\section{Synthesis of ${ }^{\mathrm{F}} \mathrm{TMSE}-\mathrm{Val}-\mathrm{CF}_{3}$-Ile-OtBu, 20c.}

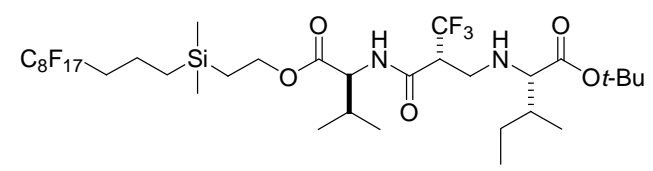

Following General Procedure F, from 19 (67 mg, $0.085 \mathrm{mmol}$ ) and isoleucine tert-butyl ester hydrochloride (19 mg, $0.085 \mathrm{mmol}$ ), $76 \mathrm{mg}$ of 20c were obtained as a white solid (92\% yield) in a 27:1 mixture of diastereoisomers as determined from its ${ }^{19} \mathrm{~F}$ NMR spectrum. NMR data for the major diastereoisomer: ${ }^{1} \mathrm{H}$ NMR (CDCl, $\left.300 \mathrm{MHz}\right) \delta 0.00$ (s, 6H), 0.53-0.59 (m, 2H), 0.79-0.90 
(m, 12H), 0.92-1.00 (m, 2H), 1.05-1.13 (m, 2H), 1.41 (s, 9H), 1.49-1.67 (m, 2H), 1.73 (br, 1H), 1.94-2.19 (m, 4H), 2.94-3.05 (m, 4H), 4.11-4.17 (m, 2H), 4.47 (dd, J= 8.7, 4.7 Hz, 1H), 7.43 (d, $J=8.7 \mathrm{~Hz}, 1 \mathrm{H}) ;{ }^{13} \mathrm{C} \mathrm{NMR}\left(\mathrm{CDCl}_{3}, 75.5 \mathrm{MHz}\right) \delta-3.6,11.6,14.8,15.1,15.4,15.9,17.6,19.1$, 25.5, 28.1, 30.8, 34.4 (t, $\left.{ }^{2} J_{\mathrm{CF}}=22.4 \mathrm{~Hz}\right), 38.5$, 45.5, 47.3, $51.1\left(\mathrm{t},{ }^{2} J_{\mathrm{CF}}=24.7 \mathrm{~Hz}\right), 57.4,63.2$, 67.1, 81.9, $124.5\left(\mathrm{t},{ }^{1} J_{\mathrm{CF}}=280.1 \mathrm{~Hz}\right), 166.3,171.9,174.6$, (the signals from the $\mathrm{C}_{8} \mathrm{~F}_{17}$ group were obscured due to their low intensity); ${ }^{19} \mathrm{~F} \mathrm{NMR}\left(\mathrm{CDCl}_{3}, 282.4 \mathrm{MHz}\right) \delta-67.4\left(\mathrm{~d}, J_{\mathrm{HF}}=8.1 \mathrm{~Hz}, 3 \mathrm{~F}\right)$, $-81.5\left(\mathrm{t},{ }^{3} J_{\mathrm{FF}}=9.8 \mathrm{~Hz}, 3 \mathrm{~F}\right),-115.1$ (br, 2F), -122.6 (br, 6F), -123.4 (m, 2F), -124.3 (m, 2F), -126.8 (m, 2F). NMR data for the minor diastereoisomer: ${ }^{19} \mathrm{~F} \mathrm{NMR}\left(\mathrm{CDCl}_{3}, 282.4 \mathrm{MHz}\right) \delta-66.3(\mathrm{~d}$, $\left.J_{\mathrm{HF}}=9.3 \mathrm{~Hz}, 3 \mathrm{~F}\right)$.

\section{Synthesis of ${ }^{F_{T}}$ TMSE-Val-CF 3 -Ala-OMe, 20d.}

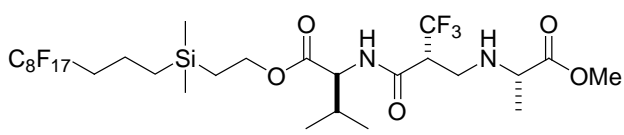

Following General Procedure F, from 19 (52 mg, $0.066 \mathrm{mmol}$ ) and alanine methyl ester hydrochloride (9 mg, $0.066 \mathrm{mmol}$ ), $52 \mathrm{mg}$ of $20 \mathrm{~d}$ were obtained as a white solid (88\% yield) in a 15:1 mixture of diastereoisomers as determined from its ${ }^{19} \mathrm{~F}$ NMR spectrum. NMR data for the major diastereoisomer: ${ }^{1} \mathrm{H}$ NMR $\left(\mathrm{CDCl}_{3}, 300 \mathrm{MHz}\right) \delta 0.01(\mathrm{~s}, 6 \mathrm{H}), 0.53-0.59(\mathrm{~m}, 2 \mathrm{H}), 0.86(\mathrm{~d}$, $J=6.9 \mathrm{~Hz}, 3 \mathrm{H}), 0.91$ (d, $J=6.9 \mathrm{~Hz}, 3 \mathrm{H}), 0.95-1.01$ (m, 2H), 1.19 (br, 1H), 1.27 (d, J= 7.2 Hz, $3 \mathrm{H}), 1.50-1.61(\mathrm{~m}, 2 \mathrm{H}), 1.94-2.09(\mathrm{~m}, 2 \mathrm{H}), 2.10-2.21(\mathrm{~m}, 1 \mathrm{H}), 2.98-3.08(\mathrm{~m}, 3 \mathrm{H}), 3.36$ (q, $J=$ $7.2 \mathrm{~Hz}, 1 \mathrm{H}), 3.68$ (br, 3H), 4.12-4.18 (m, 2H), 4.49 (dd, J= 8.7, $4.5 \mathrm{~Hz}, 1 \mathrm{H}), 7.48$ (d, J= 8.7 Hz, $1 \mathrm{H}) ;{ }^{13} \mathrm{C} \mathrm{NMR}\left(\mathrm{CDCl}_{3}, 75.5 \mathrm{MHz}\right) \delta-4.3,13.9,14.2,15.0,16.6,18.1,22.5,29.9,35.5\left(\mathrm{t},{ }^{2} J_{\mathrm{CF}}=\right.$ 22.1 Hz), 43.5, 49.8 (q, ${ }^{2} J_{\mathrm{CF}}=25.2 \mathrm{~Hz}$ ), 51.2, 56.2, 56.6, 62.4, 123.5 (q, ${ }^{1} J_{\mathrm{CF}}=280.0 \mathrm{~Hz}$ ), 165.2 , 170.0, 174.9, (the signals from the $\mathrm{C}_{8} \mathrm{~F}_{17}$ group were obscured due to their low intensity); ${ }^{19} \mathrm{~F}$ $\operatorname{NMR}\left(\mathrm{CDCl}_{3}, 282.4 \mathrm{MHz}\right) \delta-66.6\left(\mathrm{~d}, J_{\mathrm{HF}}=8.5 \mathrm{~Hz}, 3 \mathrm{~F}\right),-80.7\left(\mathrm{t},{ }^{3} J_{\mathrm{FF}}=11.3 \mathrm{~Hz}, 3 \mathrm{~F}\right),-114.4(\mathrm{br}$, 2F), -121.8 (br, 6F), -122.7 (br, 2F), -123.6 (br, 2F), -126.0 (br, 2F). NMR data for the minor diastereoisomer: ${ }^{19} \mathrm{~F} \mathrm{NMR}\left(\mathrm{CDCl}_{3}, 282.4 \mathrm{MHz}\right) \delta-65.6\left(\mathrm{~d}, J_{\mathrm{HF}}=8.4 \mathrm{~Hz}, 3 \mathrm{~F}\right)$. 


\section{Synthesis of BnO-Val-CF - Val-OBn, 1a.}

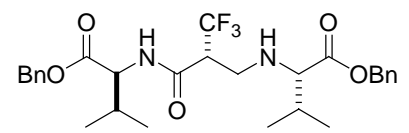

Following General Procedure B, from 20a (17 mg, $0.017 \mathrm{mmol}), 7 \mathrm{mg}$ of 1a were obtained as a white solid (77\% yield). Its physical and spectroscopic data matched those previously reported. ${ }^{2}$

\section{Synthesis of BnO-Val-CF - Leu-OBn, 1 b.}

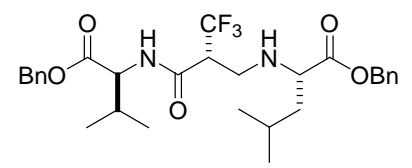

Following General Procedure B, from 20b (38 mg, $0.037 \mathrm{mmol}$ ), $14.5 \mathrm{mg}$ of $\mathbf{1 b}$ were obtained as a white solid (70\% yield). Its physical and spectroscopic data matched those previously reported.

\section{Synthesis of BnO-Val-CF 3 -Ala-OMe, $1 d$.}<smiles>COC(=O)[C@H](C)NCC(C)([Si])C(=O)NC(C(=O)OCc1ccccc1)C(C)C</smiles>

Following General Procedure B, from 20d (25 mg, $0.028 \mathrm{mmol}$ ), $10 \mathrm{mg}$ of $\mathbf{1 d}$ were obtained as a white solid (81\% yield). Its physical and spectroscopic data matched those previously reported. ${ }^{2}$

${ }^{2}$ Sani, M.; Bruché, L.; Chiva, G.; Fustero, S.; Piera, J.; Volonterio, A.; Zanda, M. Angew. Chem. Int. Ed. 2003, 42, 2060-2063. 


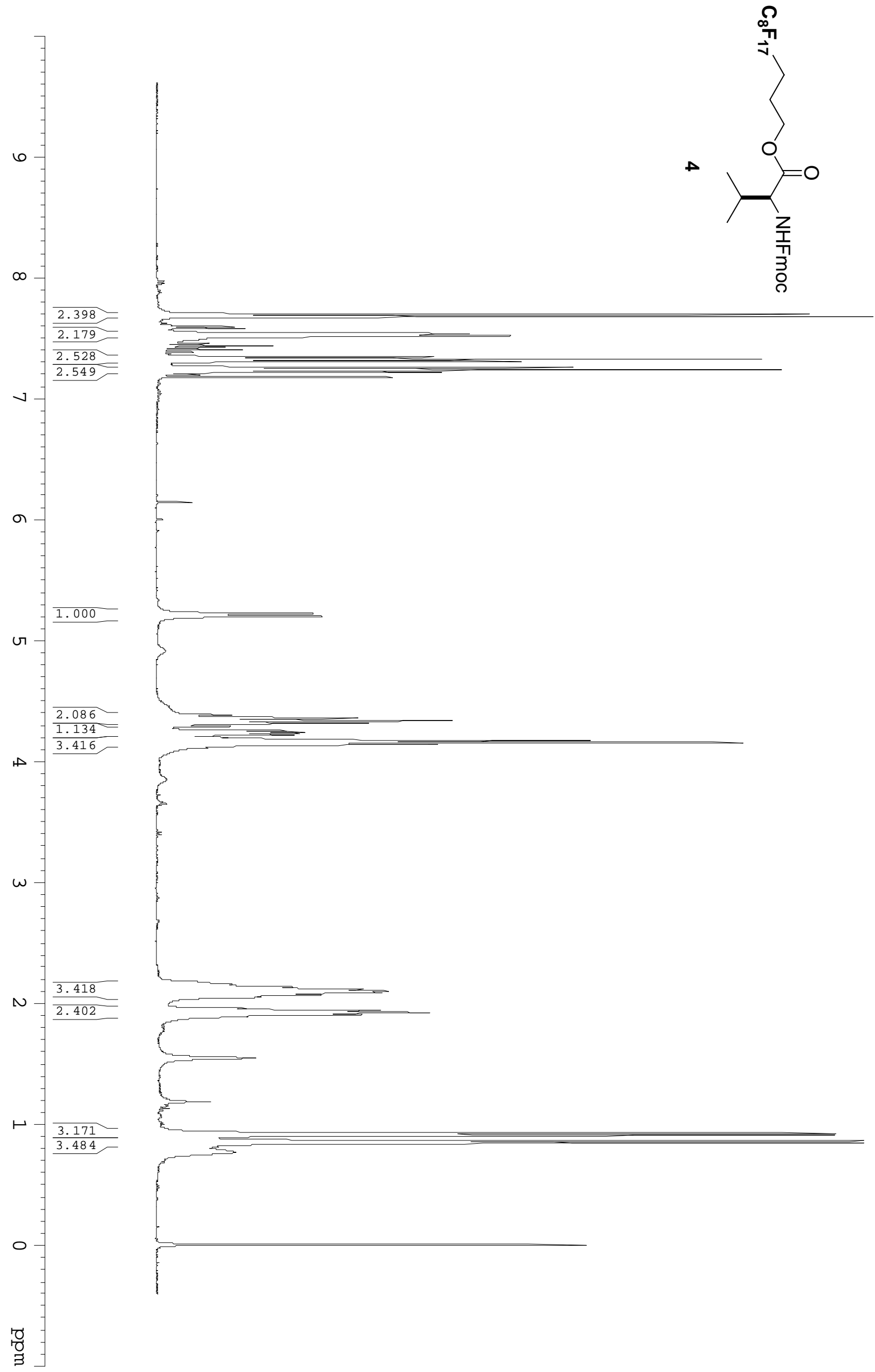




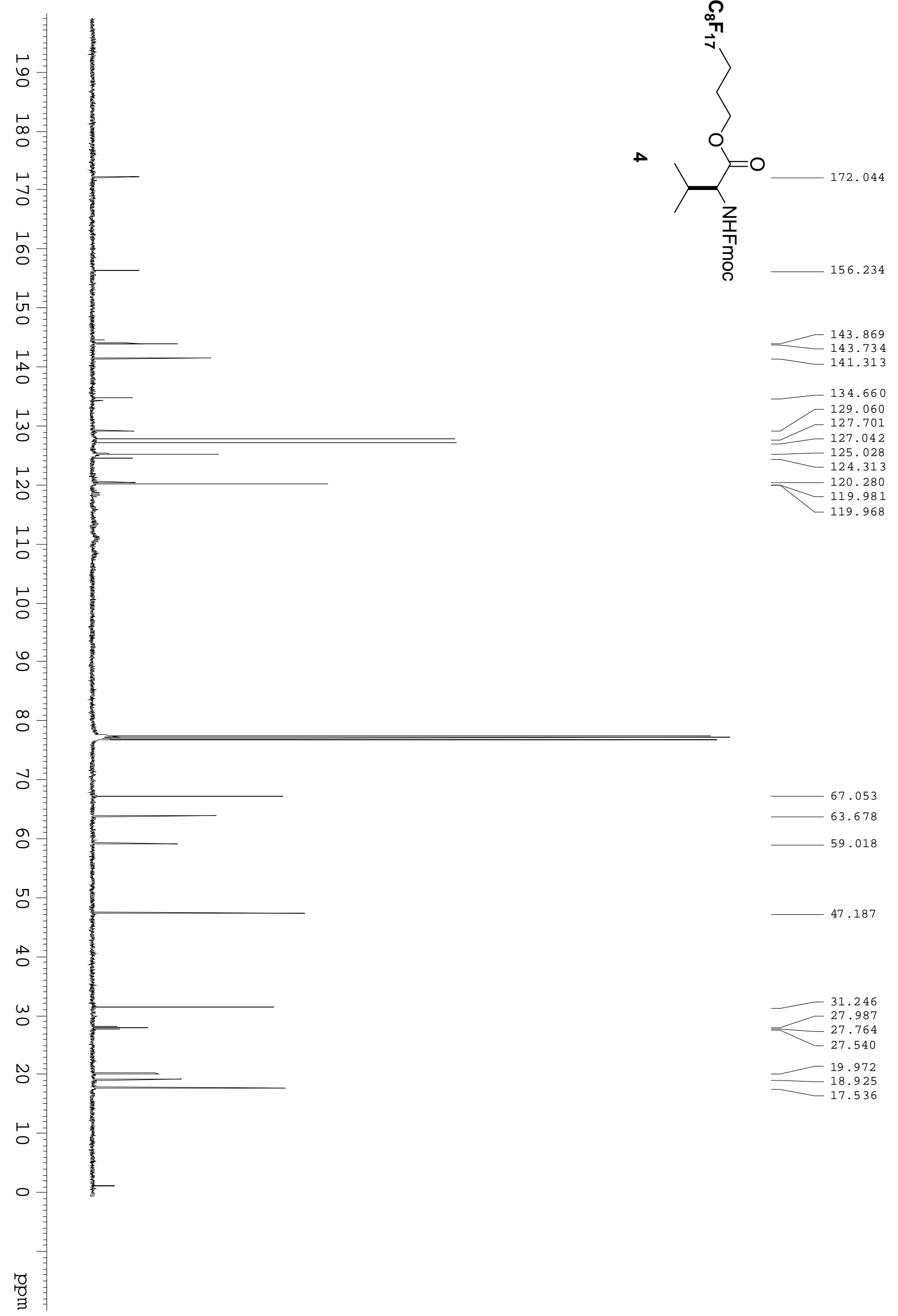



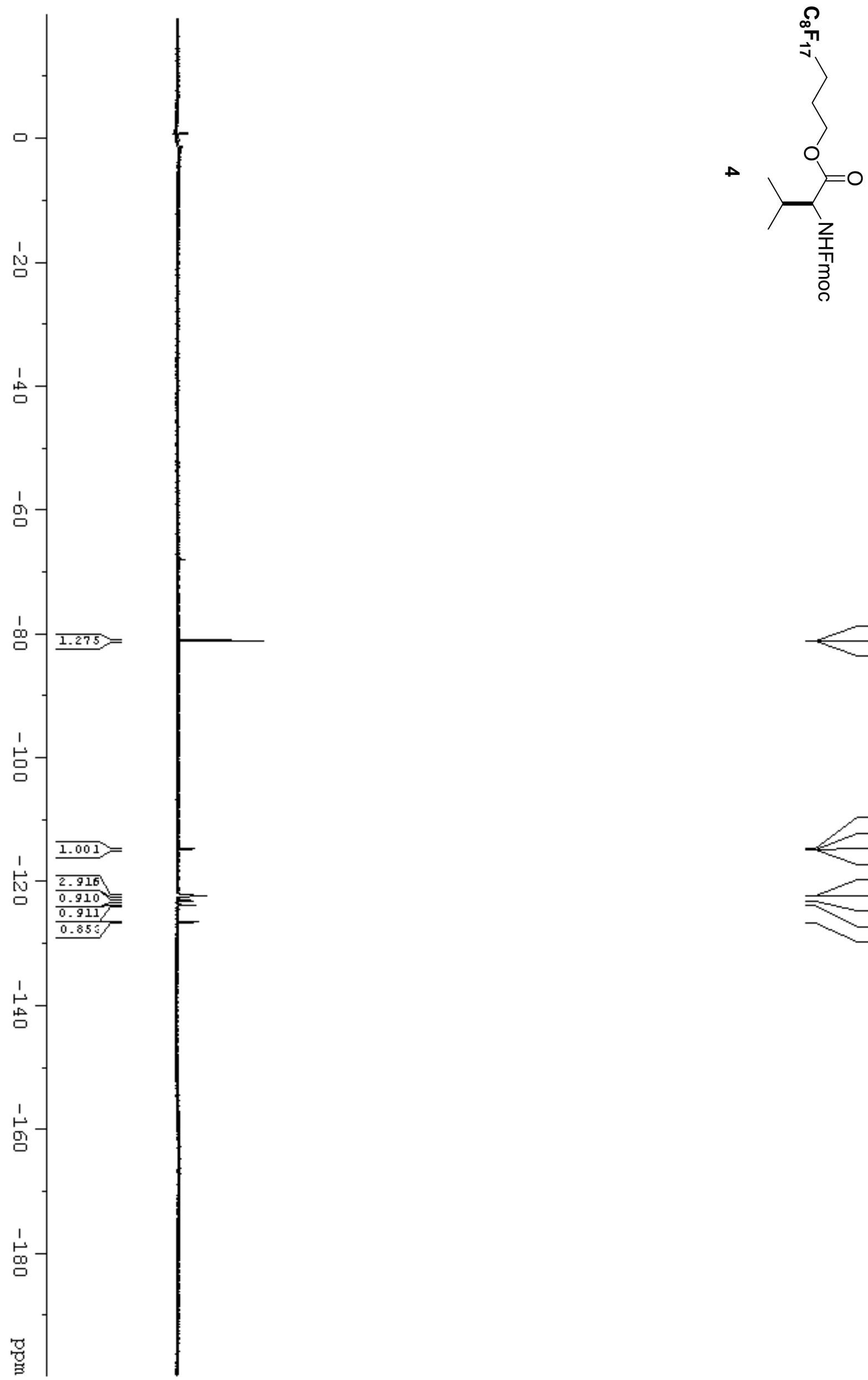

$-81.189$

$-81.224$

$-81.260$

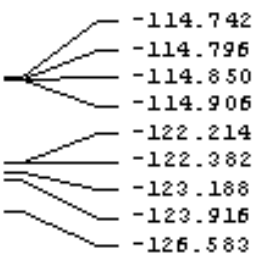



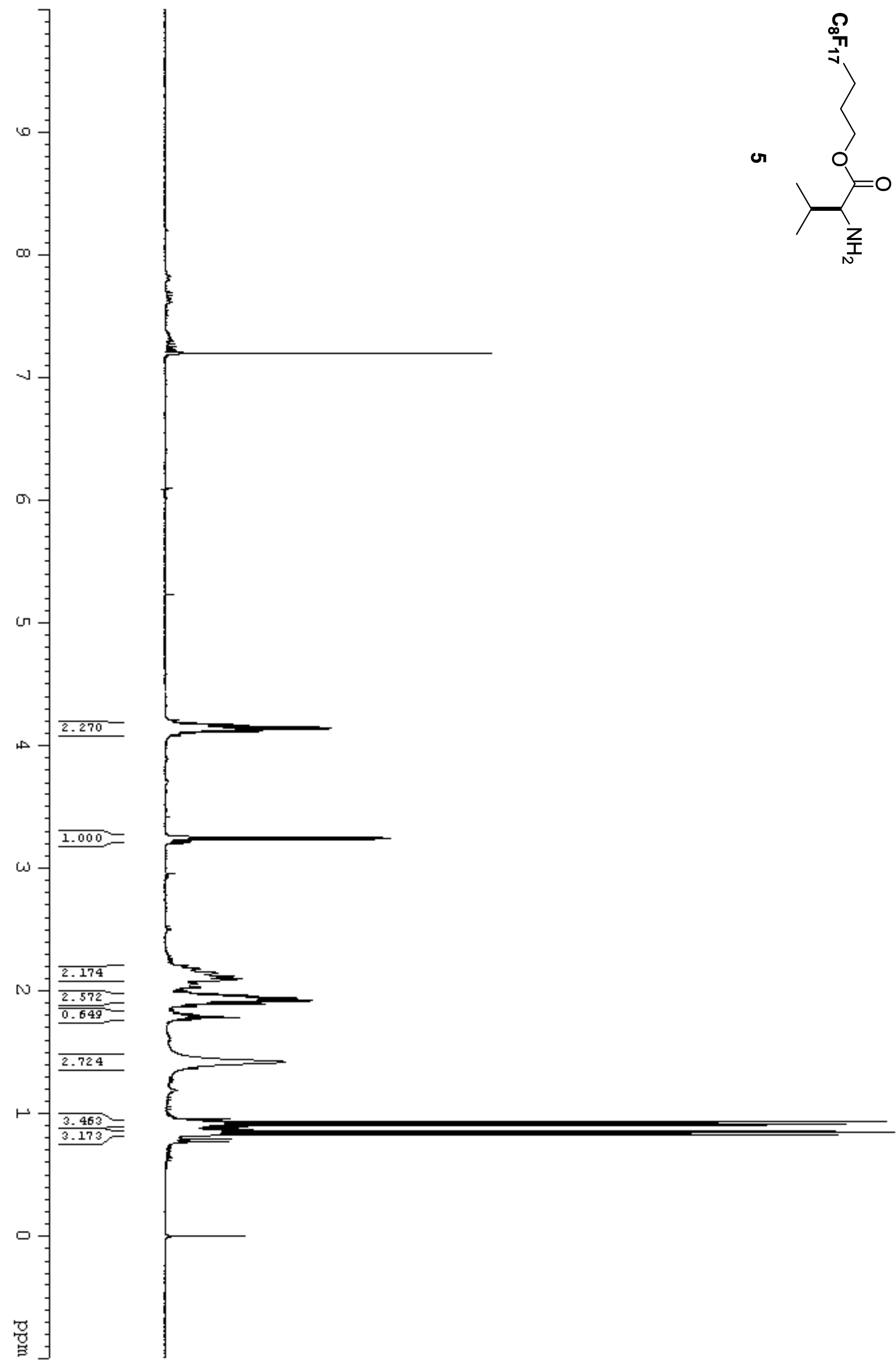


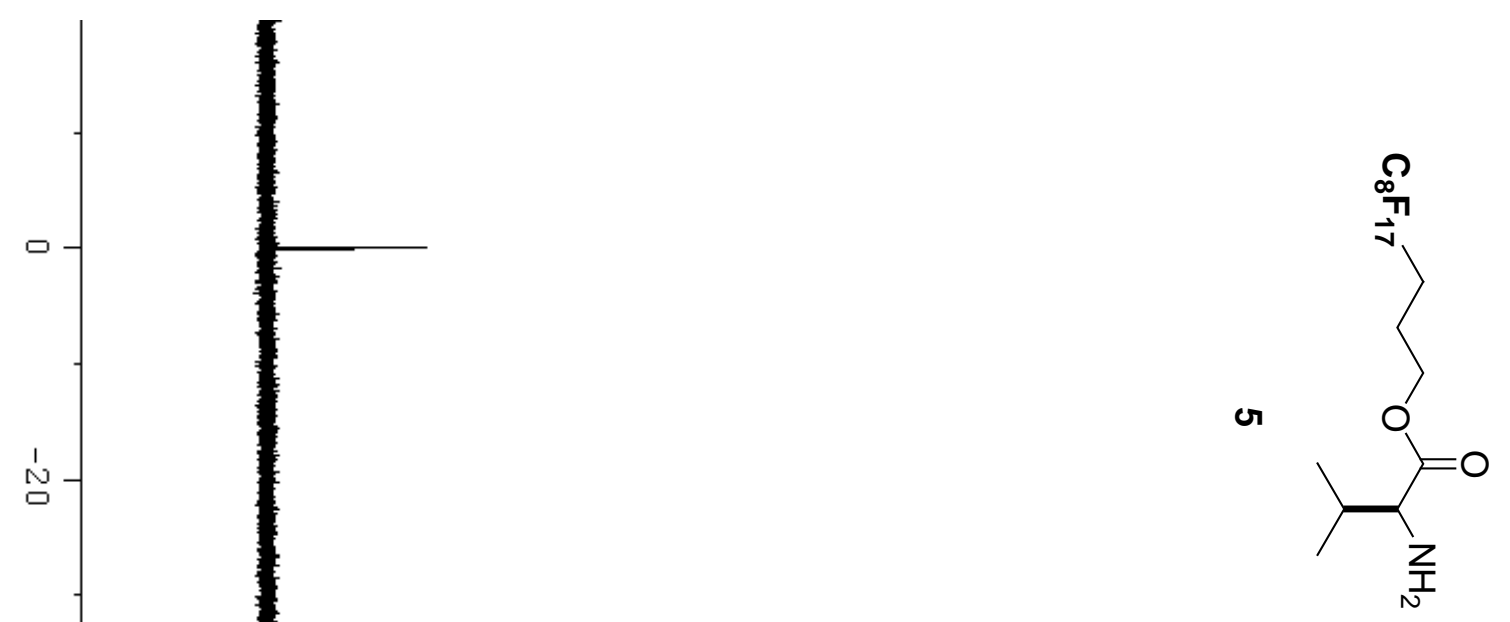

品

욤

$90-\frac{}{3.000}=$
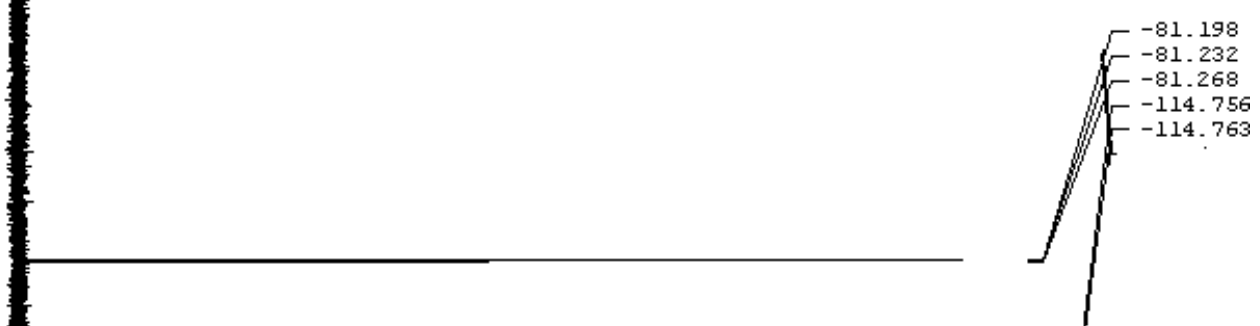

占

$\stackrel{\stackrel{\bullet}{口}}{\bullet}-\frac{\frac{2.316}{6.639}}{\frac{2.328}{2.340}=}$
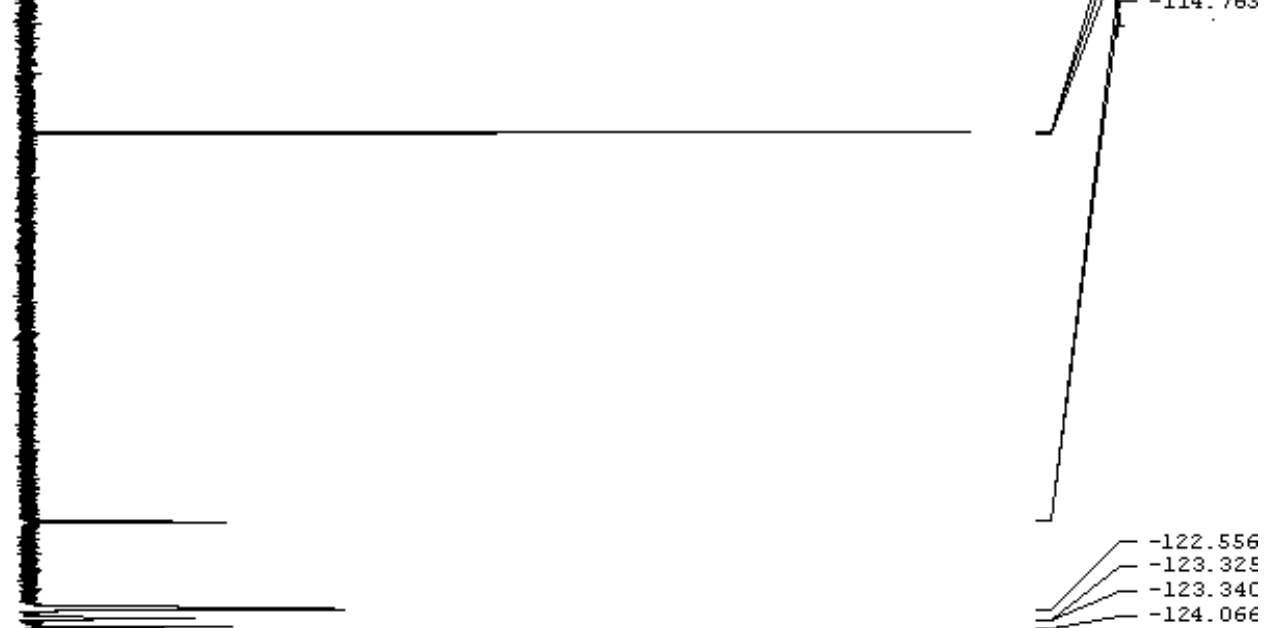

$-122.556$

$-123.325$

$-124.06 \epsilon$

-124.074
-124.084

$-124.114$

$-126.502$

$\stackrel{!}{\stackrel{5}{口}}$

占

马

昜 


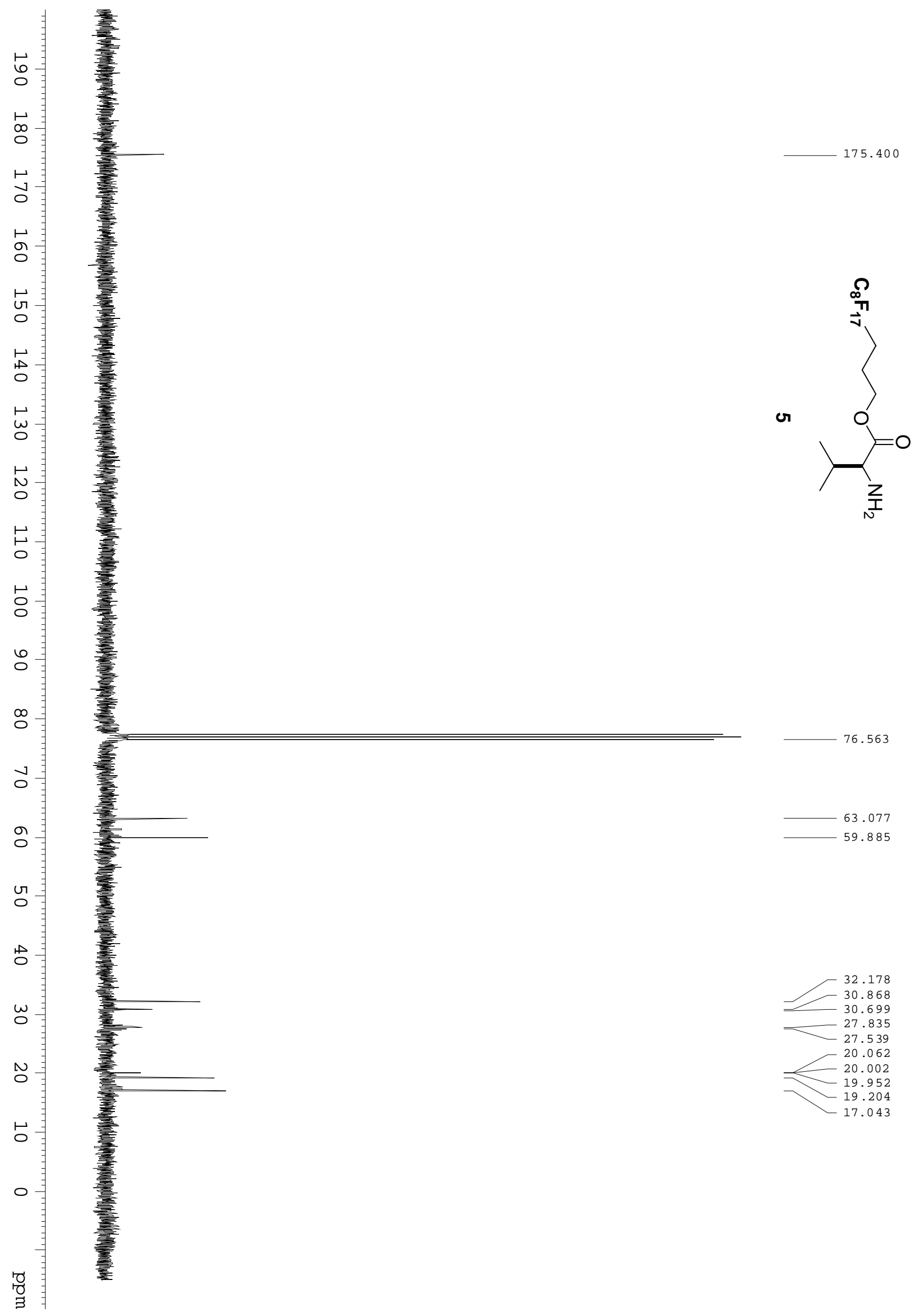




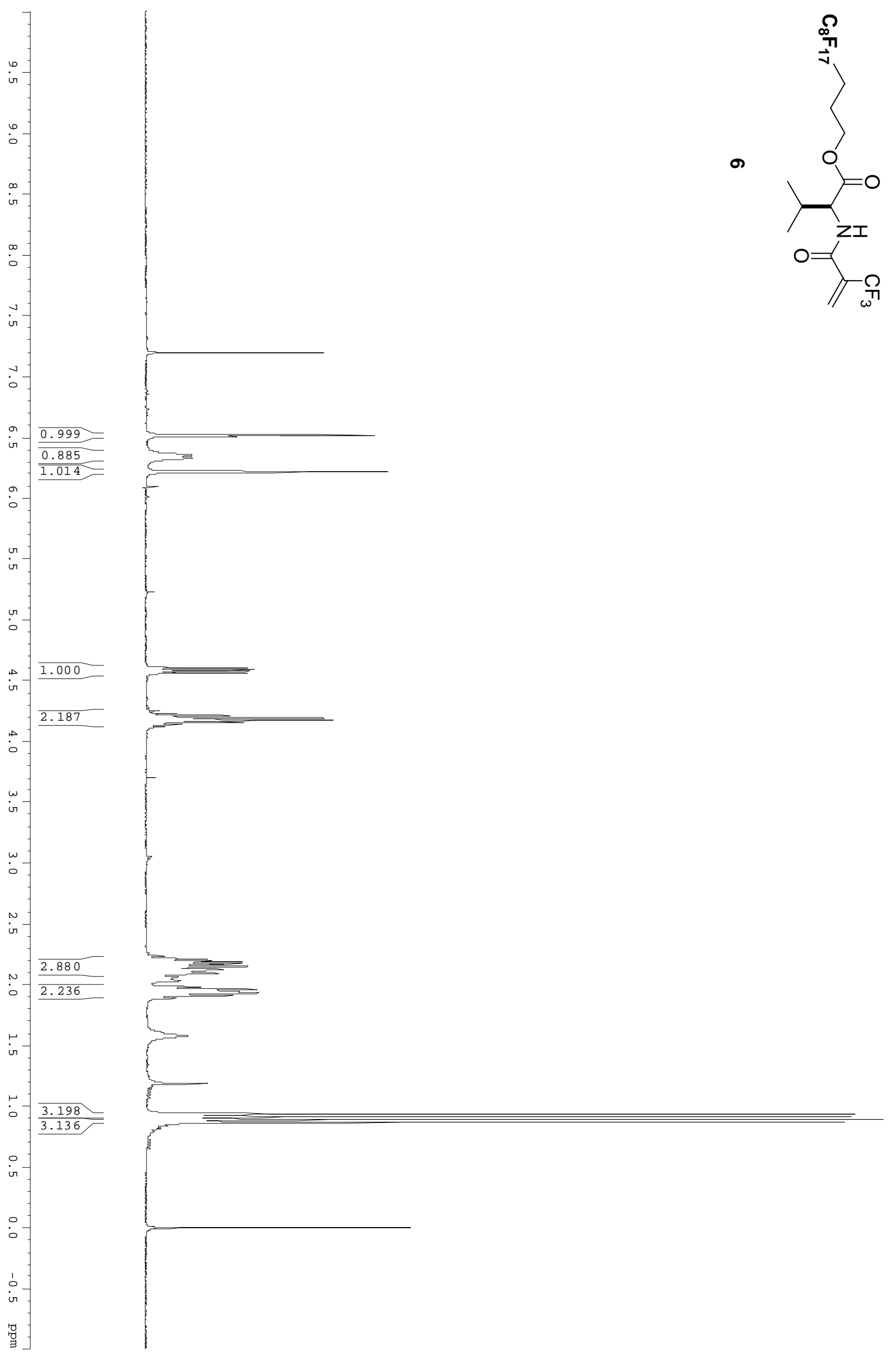




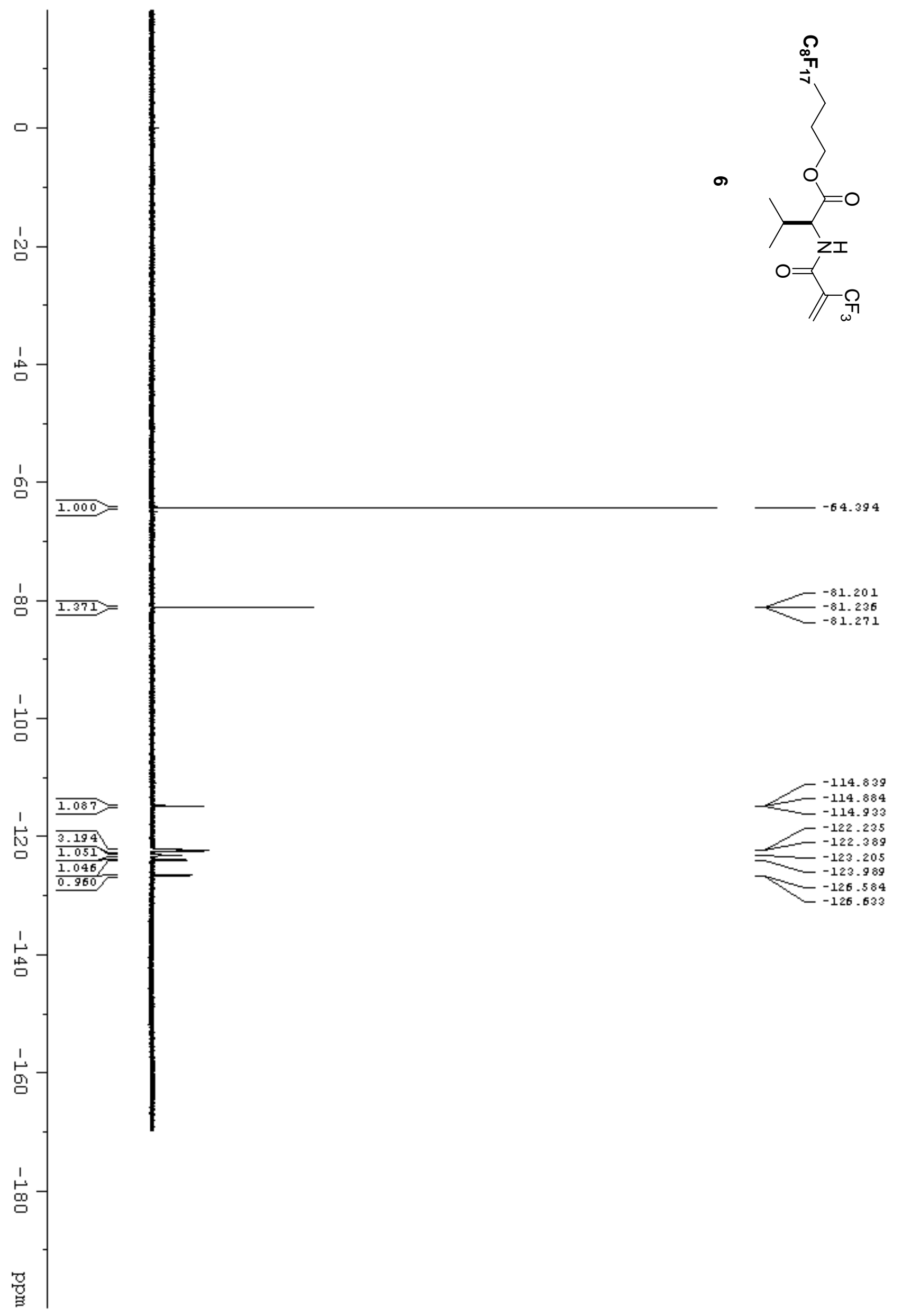




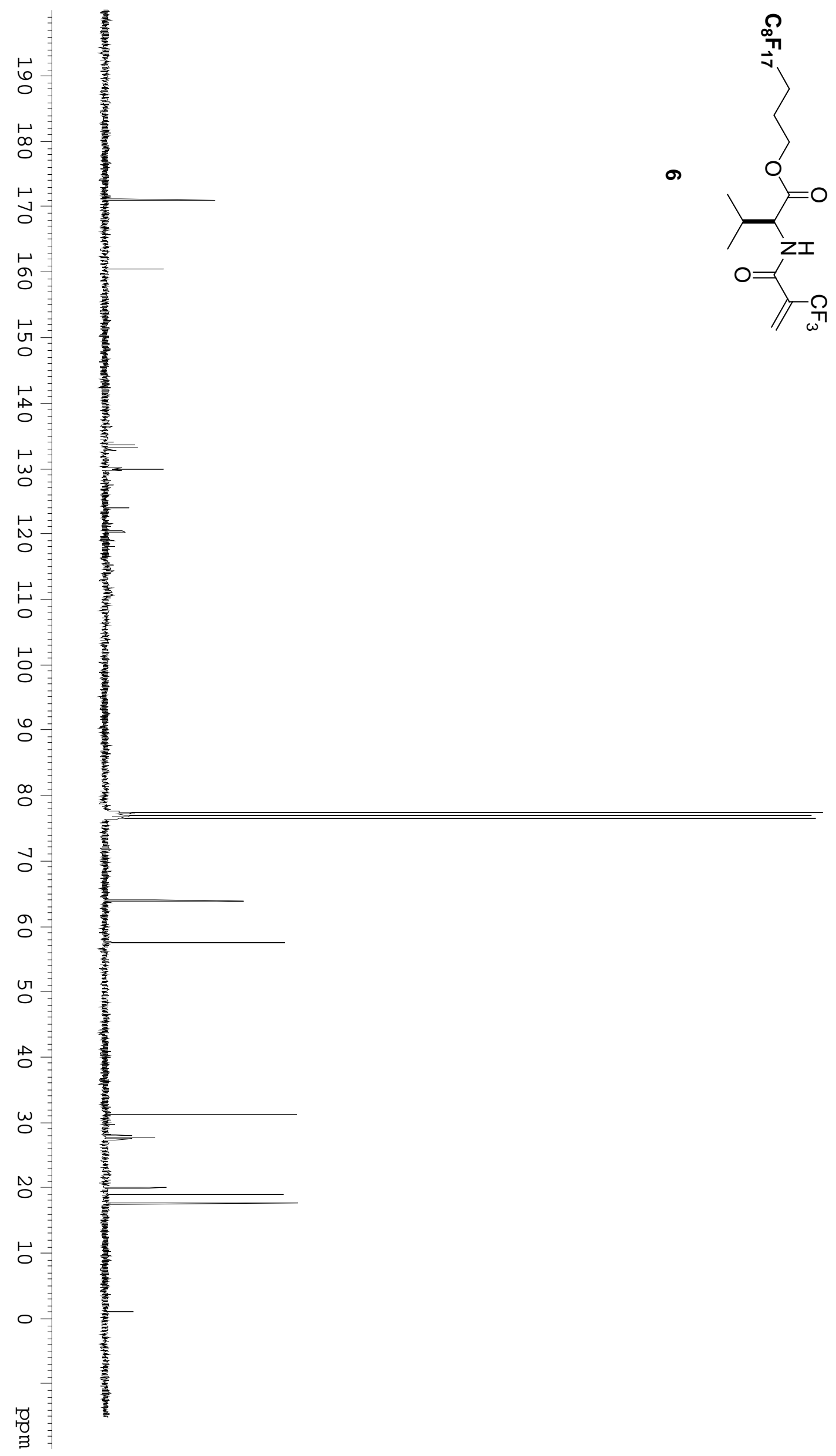

63.902

57.533

31.233

28.189

28.038

27.737

27.439

19.936

19.886

18.863

17.526 


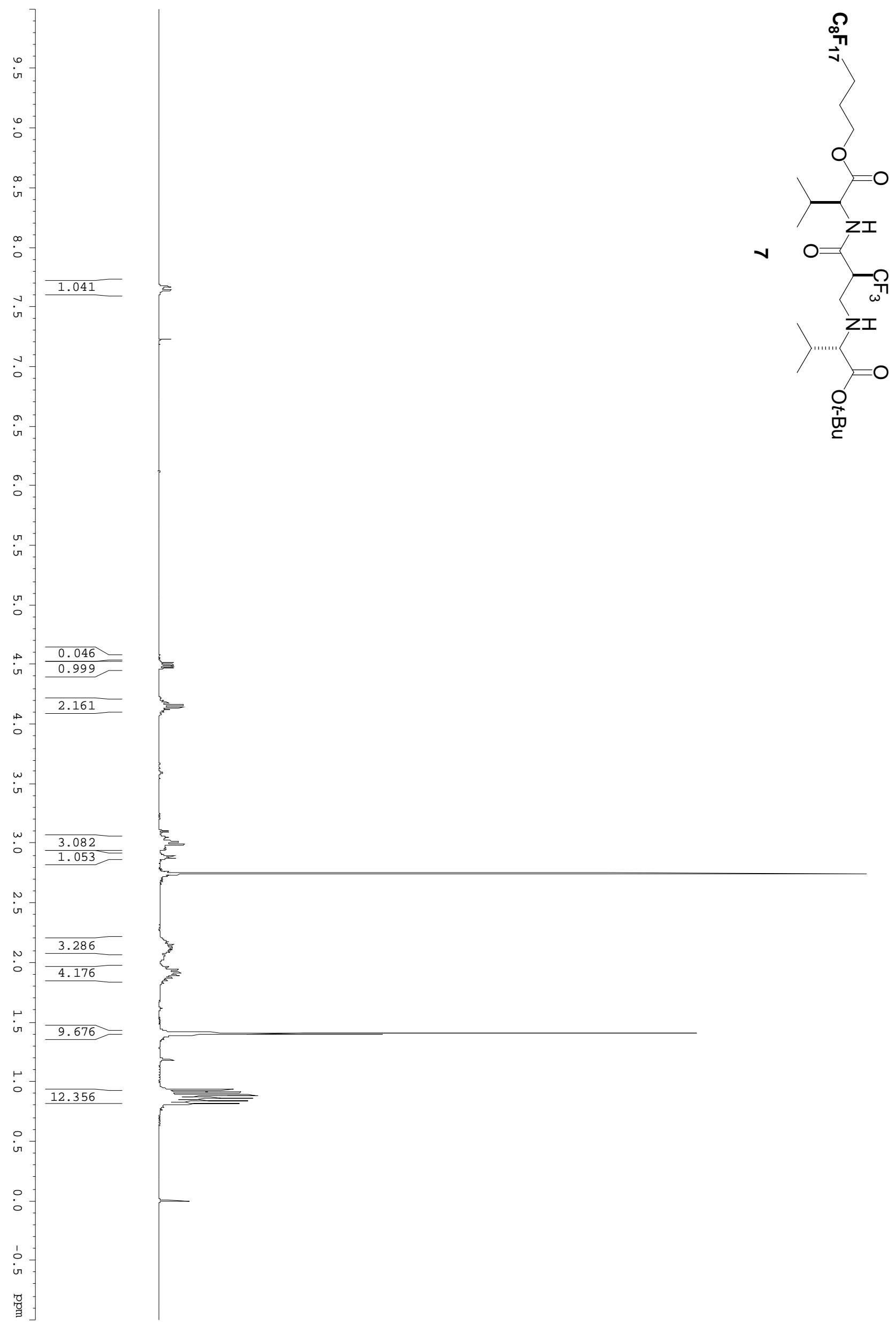




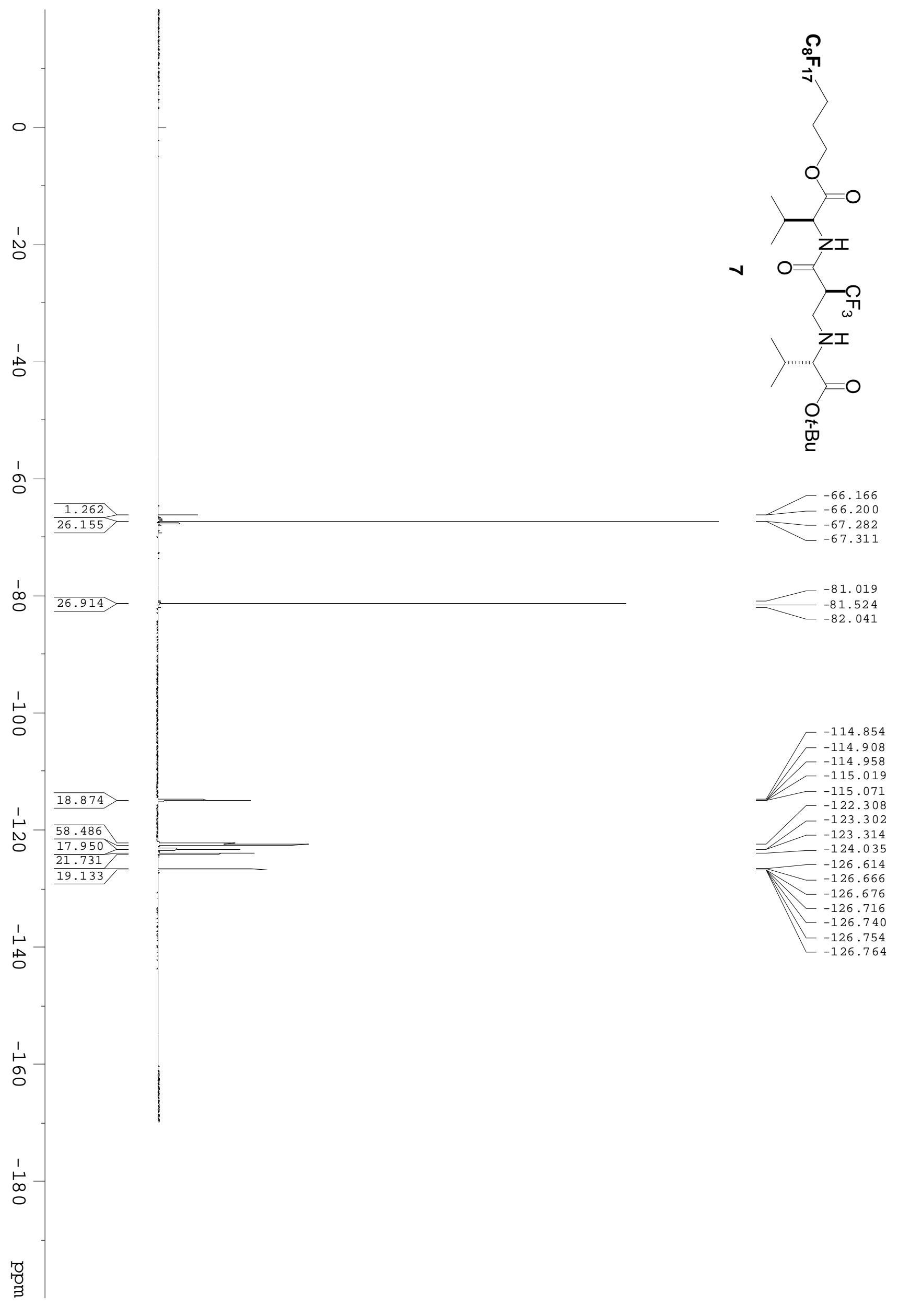




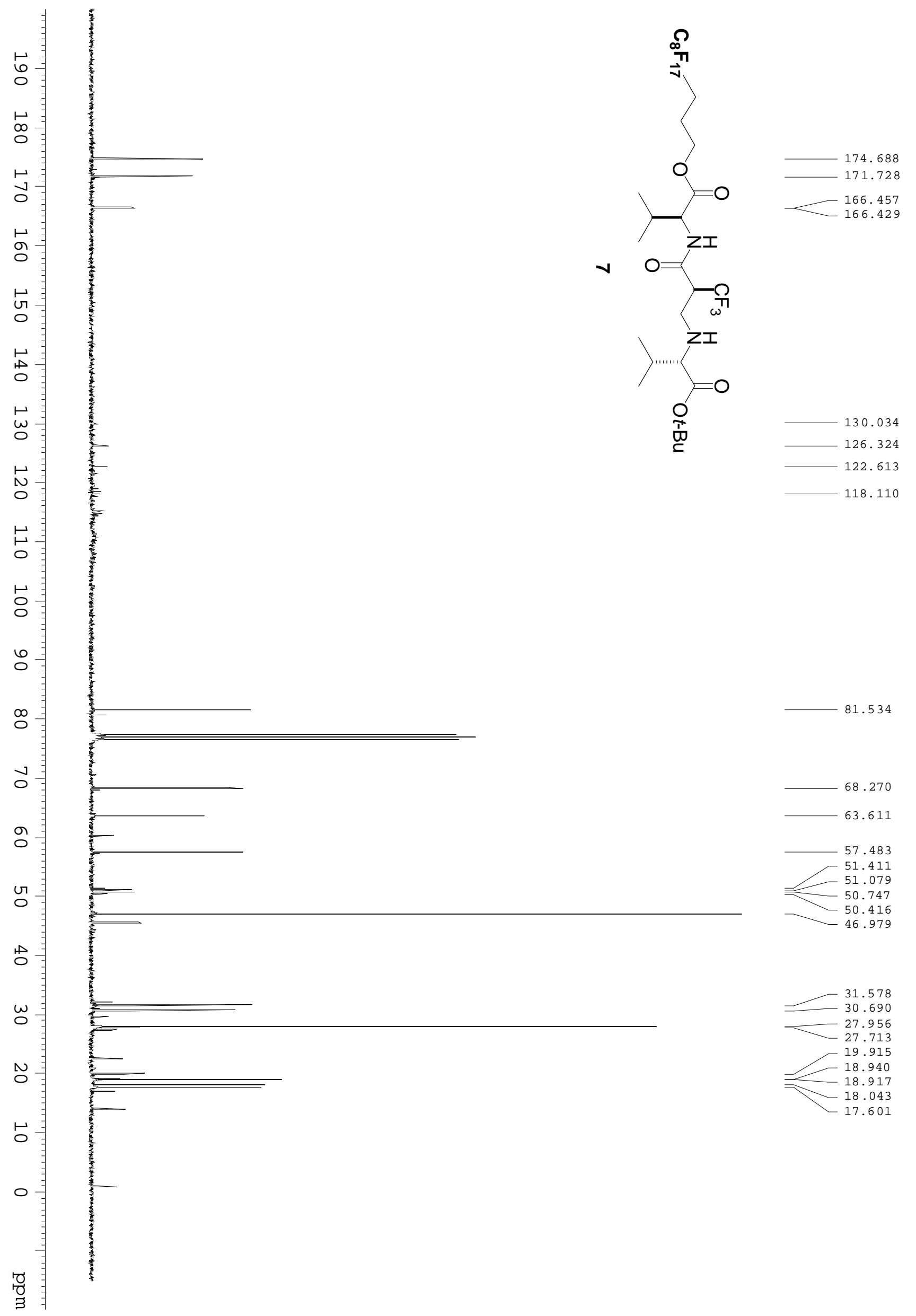




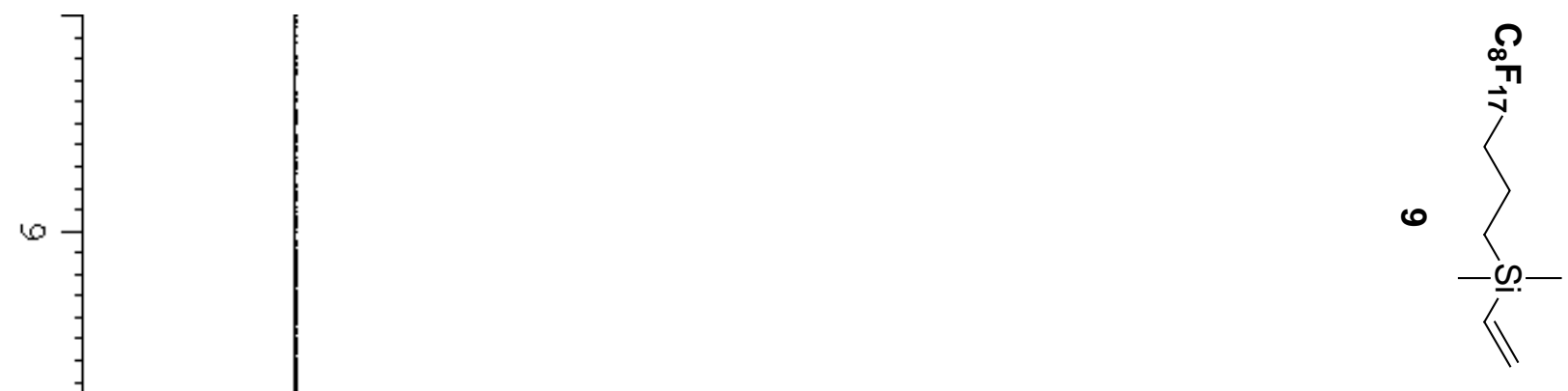

$\infty$

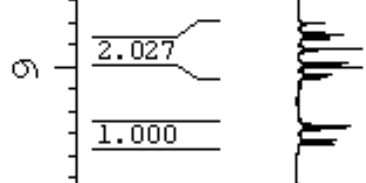

ज 


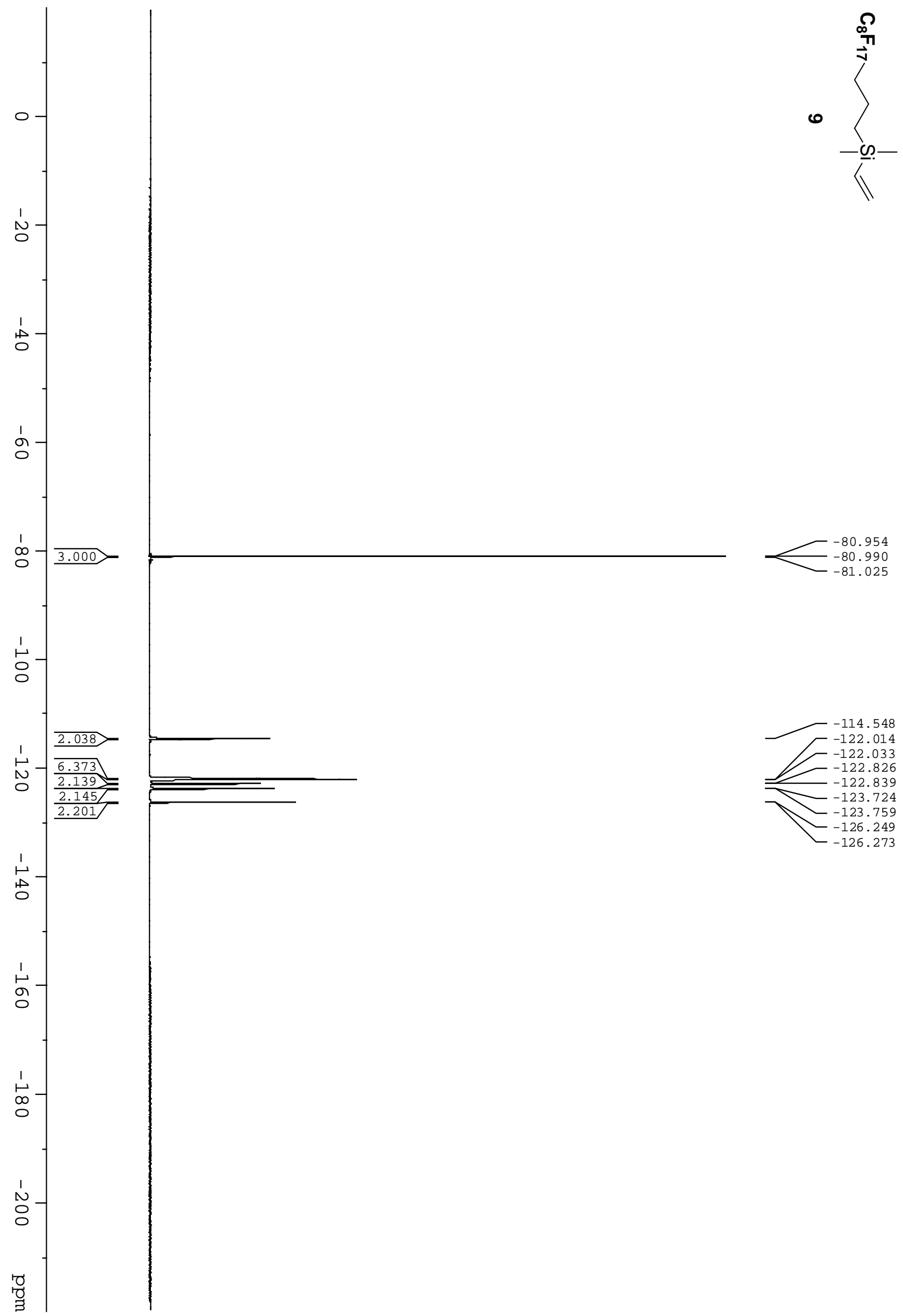




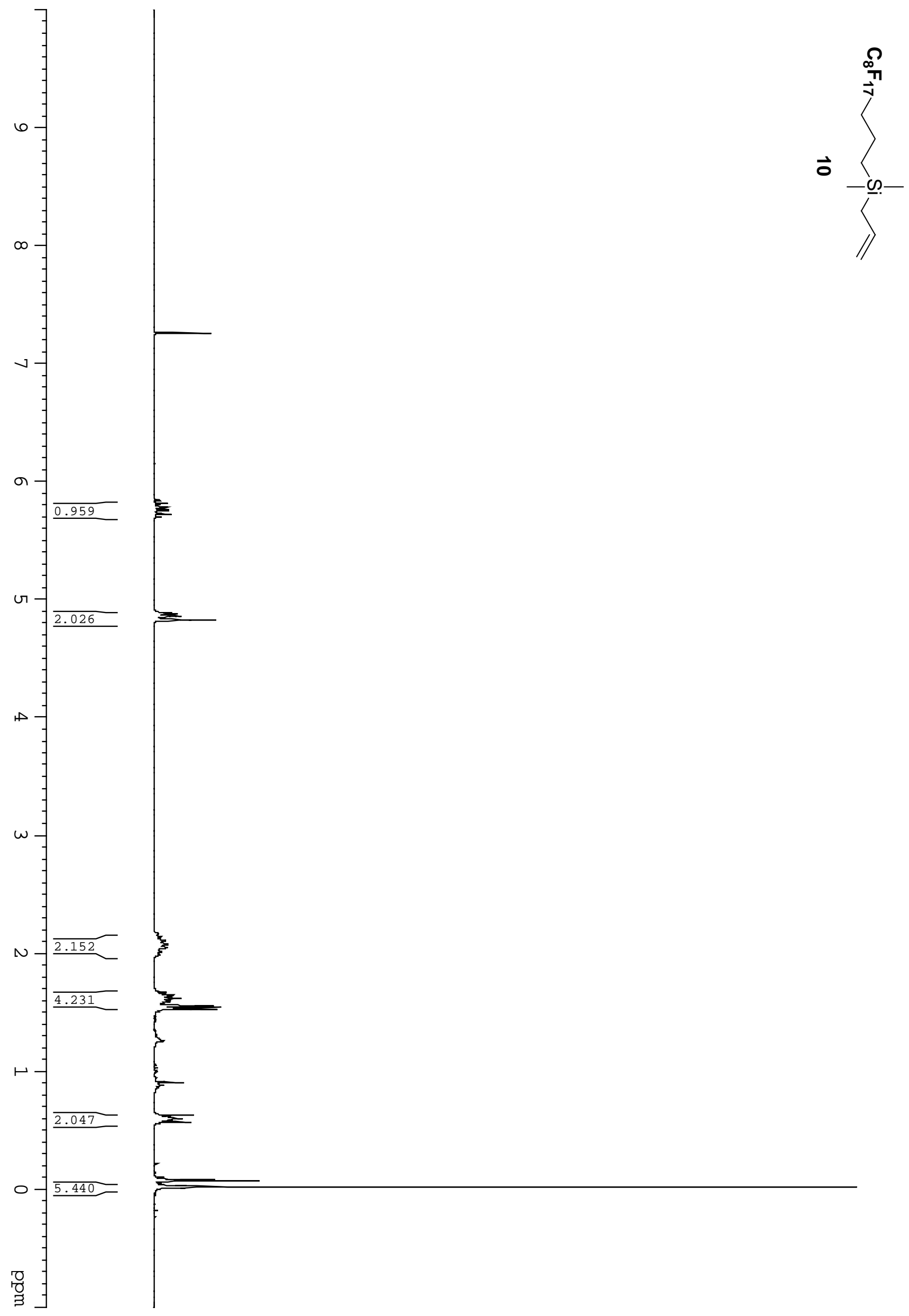




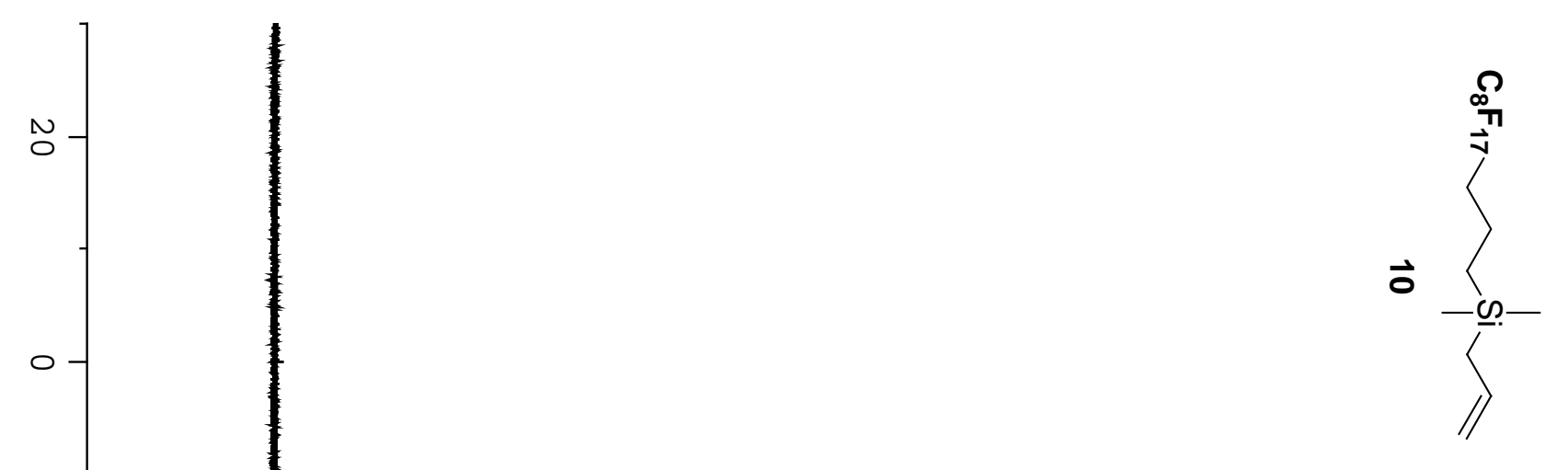

กั

๑

๑

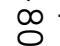

$3.002>$

$-81.222$

$-81.257$

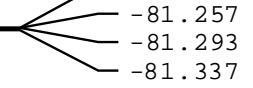

$\stackrel{\odot}{\odot}$

$\underline{2.121}=$

N

$\frac{6.225}{2.063}$

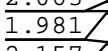

2.157

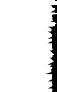
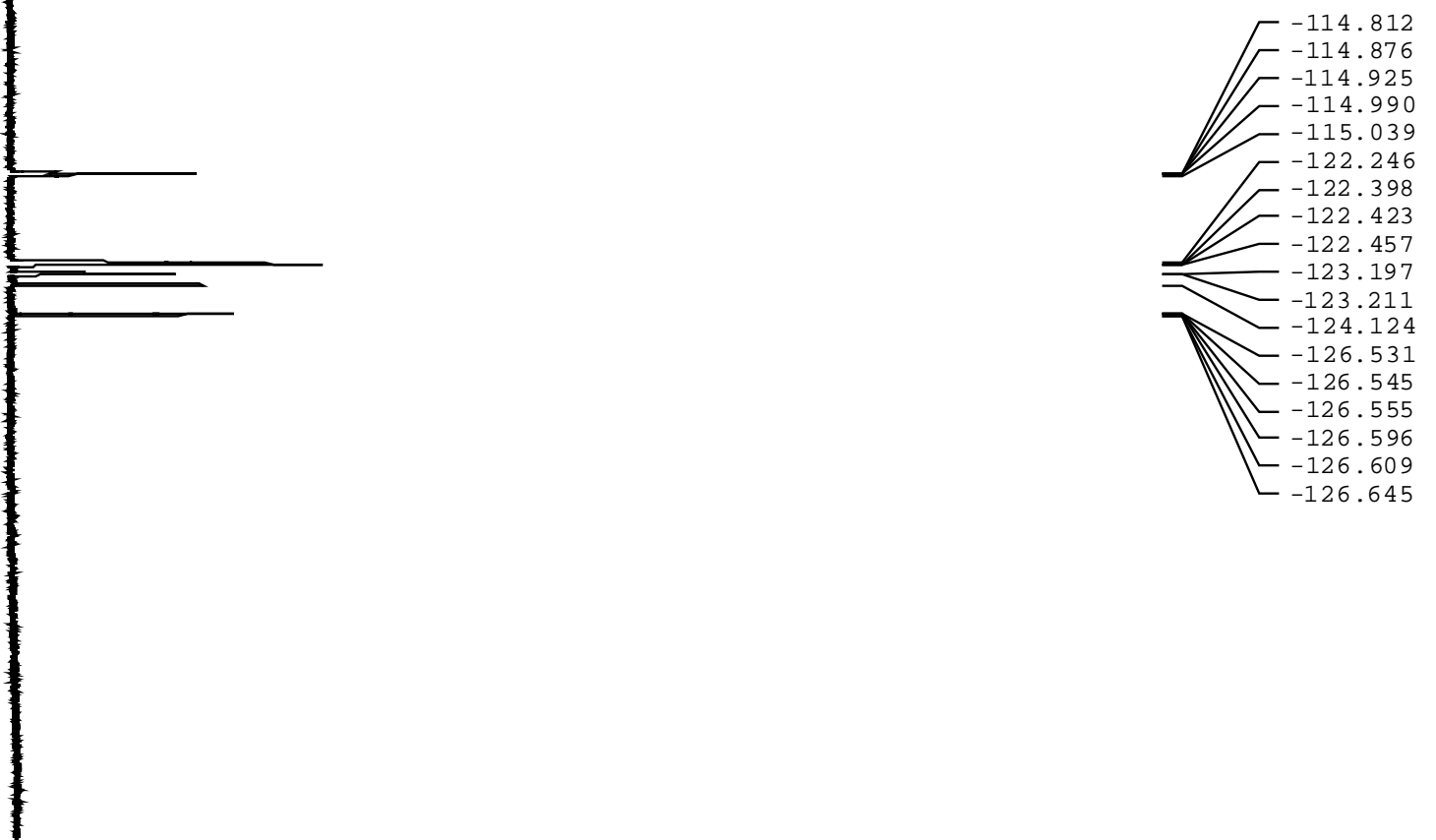

$\stackrel{\stackrel{⿱}{*}}{\stackrel{\oplus}{\odot}}$

몰$$
\text { , }
$$ 

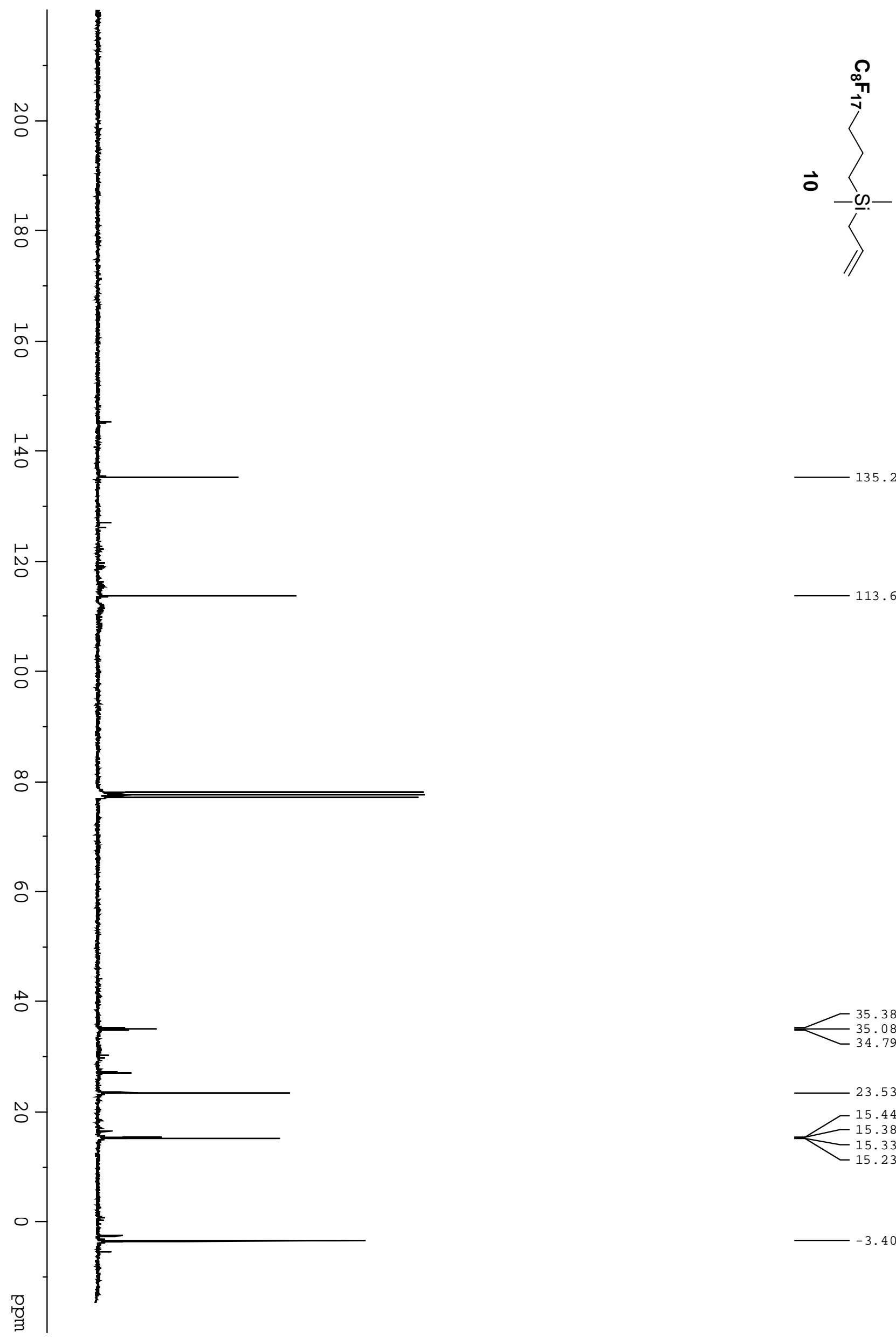

135.203

113.637

35.382

23.538

15.441

15.389

15.333

$-15.237$

$-3.403$ 


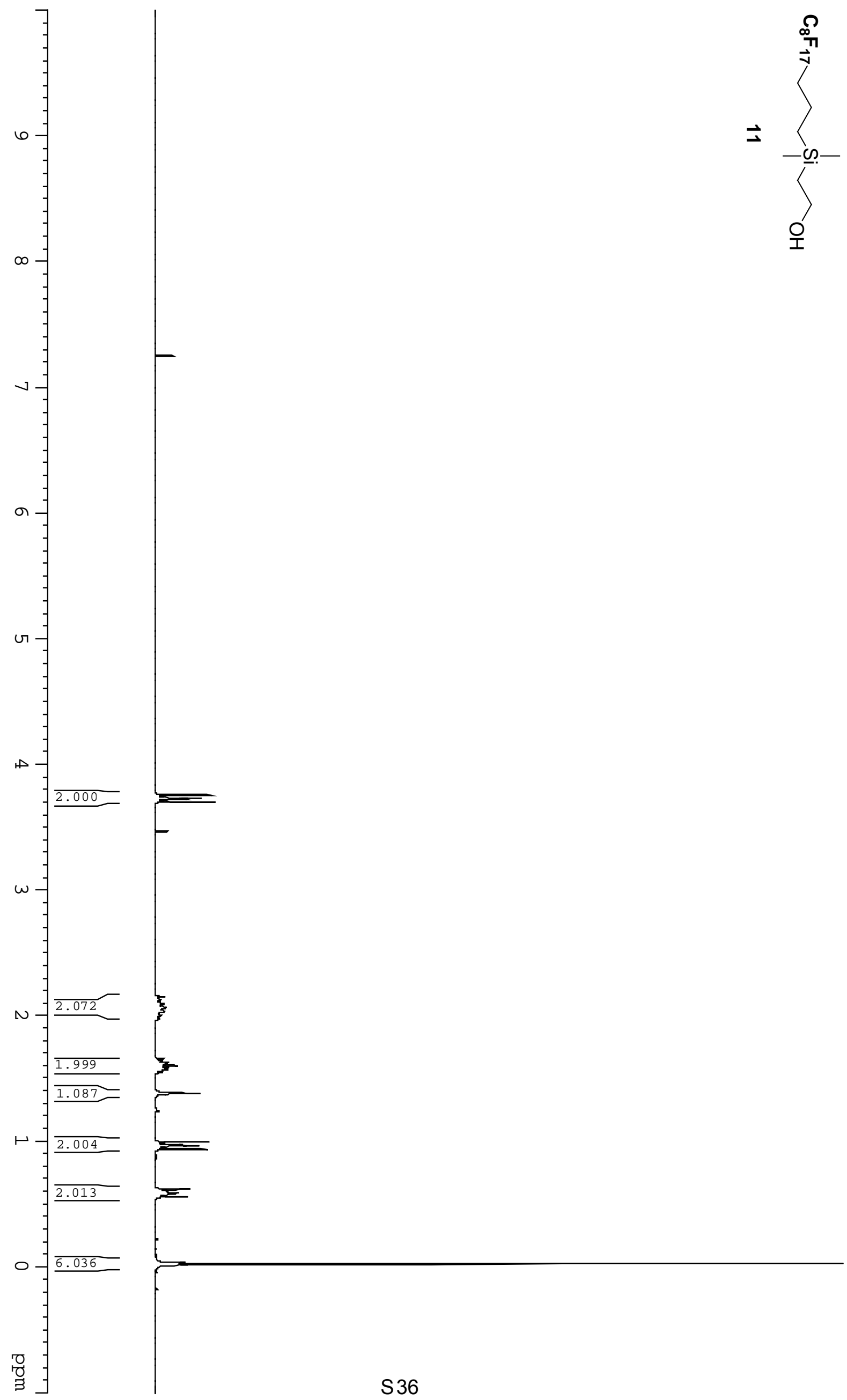




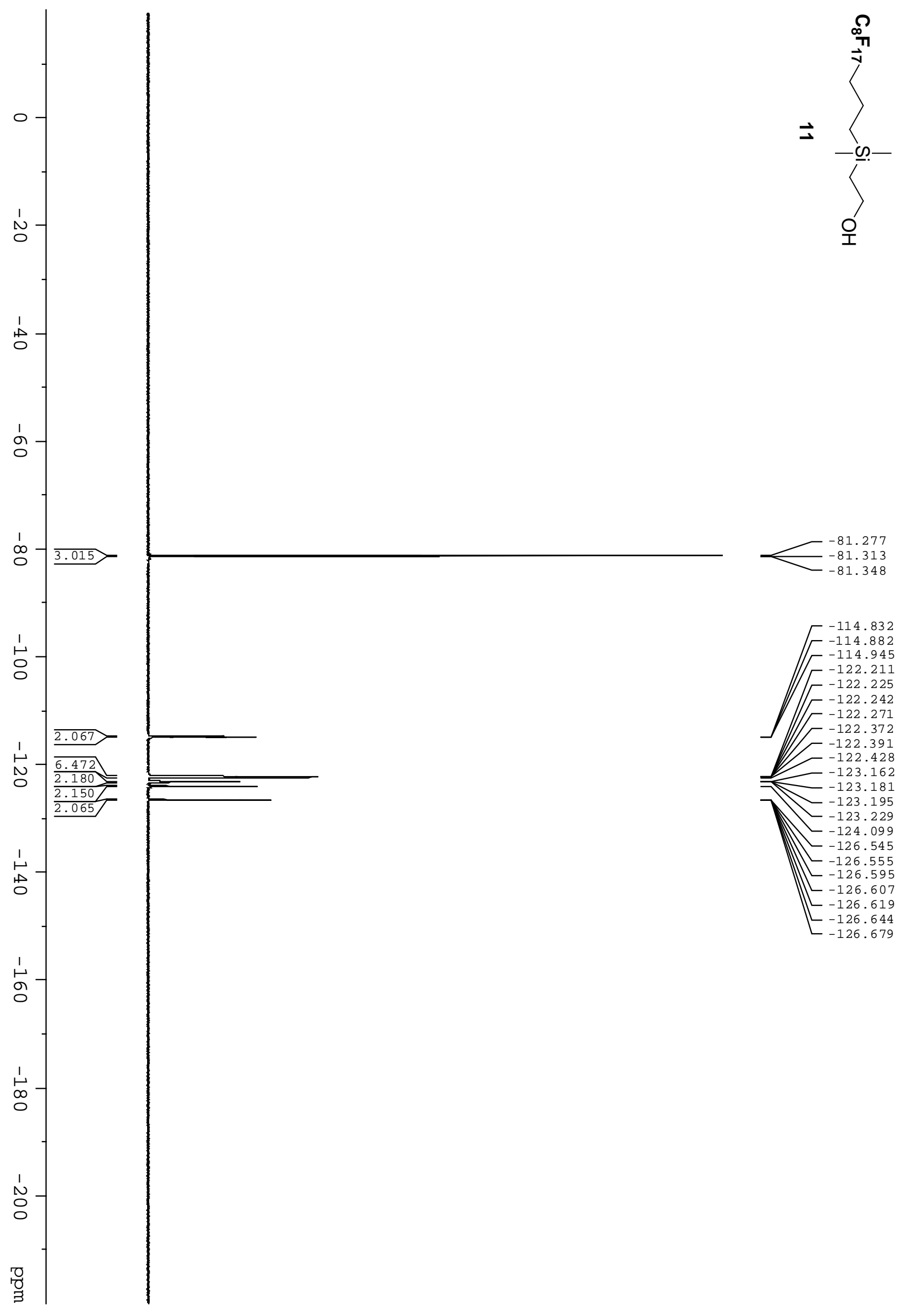




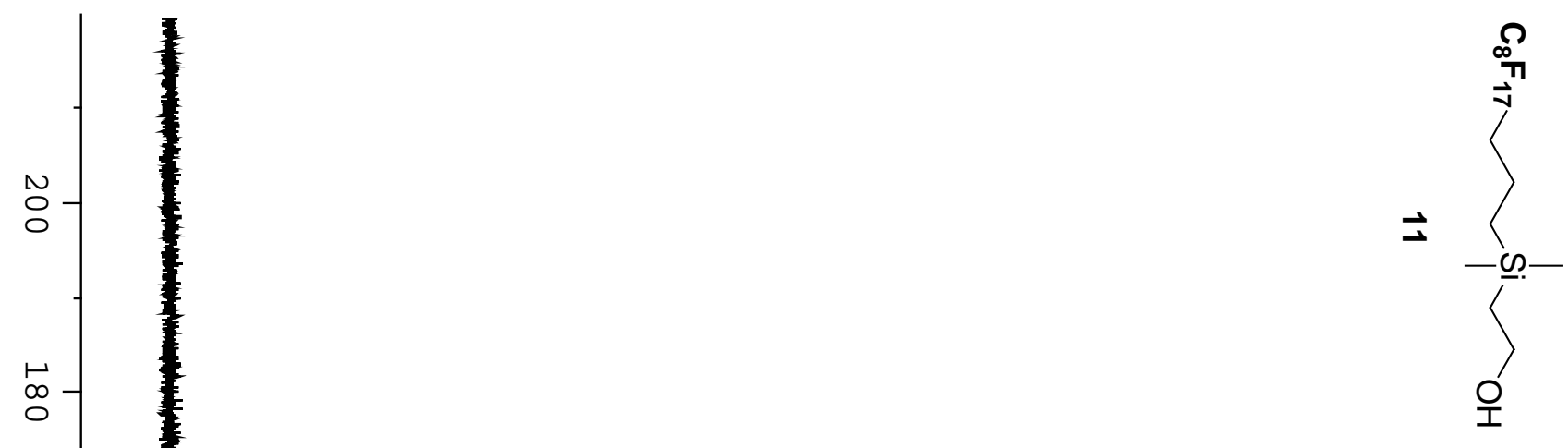

คั

$\stackrel{\leftrightarrow}{\circ}$

กั

๑ே

๑-

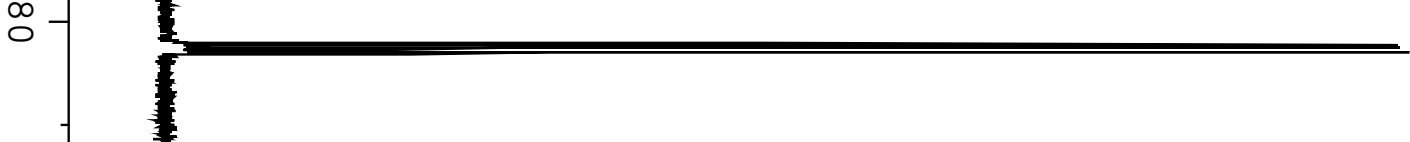

๑-

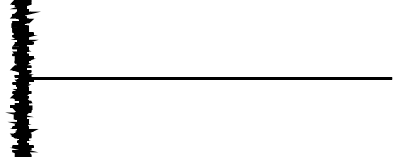

$\stackrel{\circ}{\circ}$

๑

$\sqrt{3}$

๑

60.224
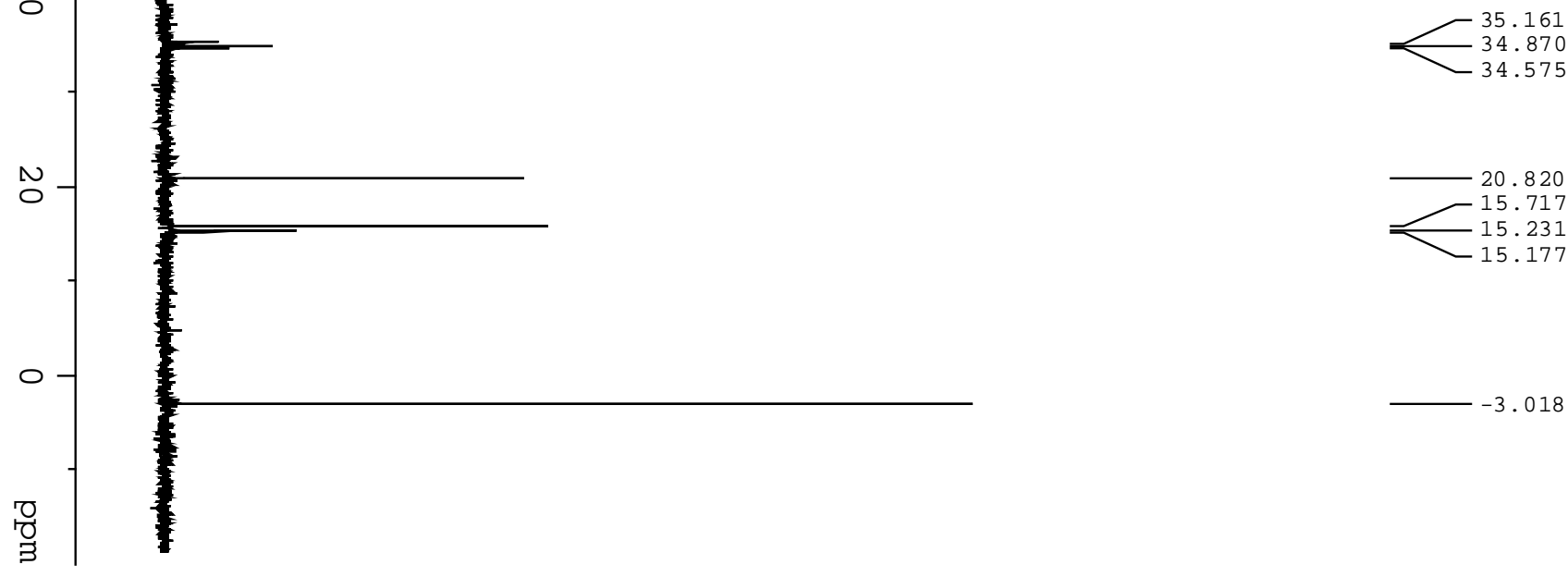

$-3.018$ 


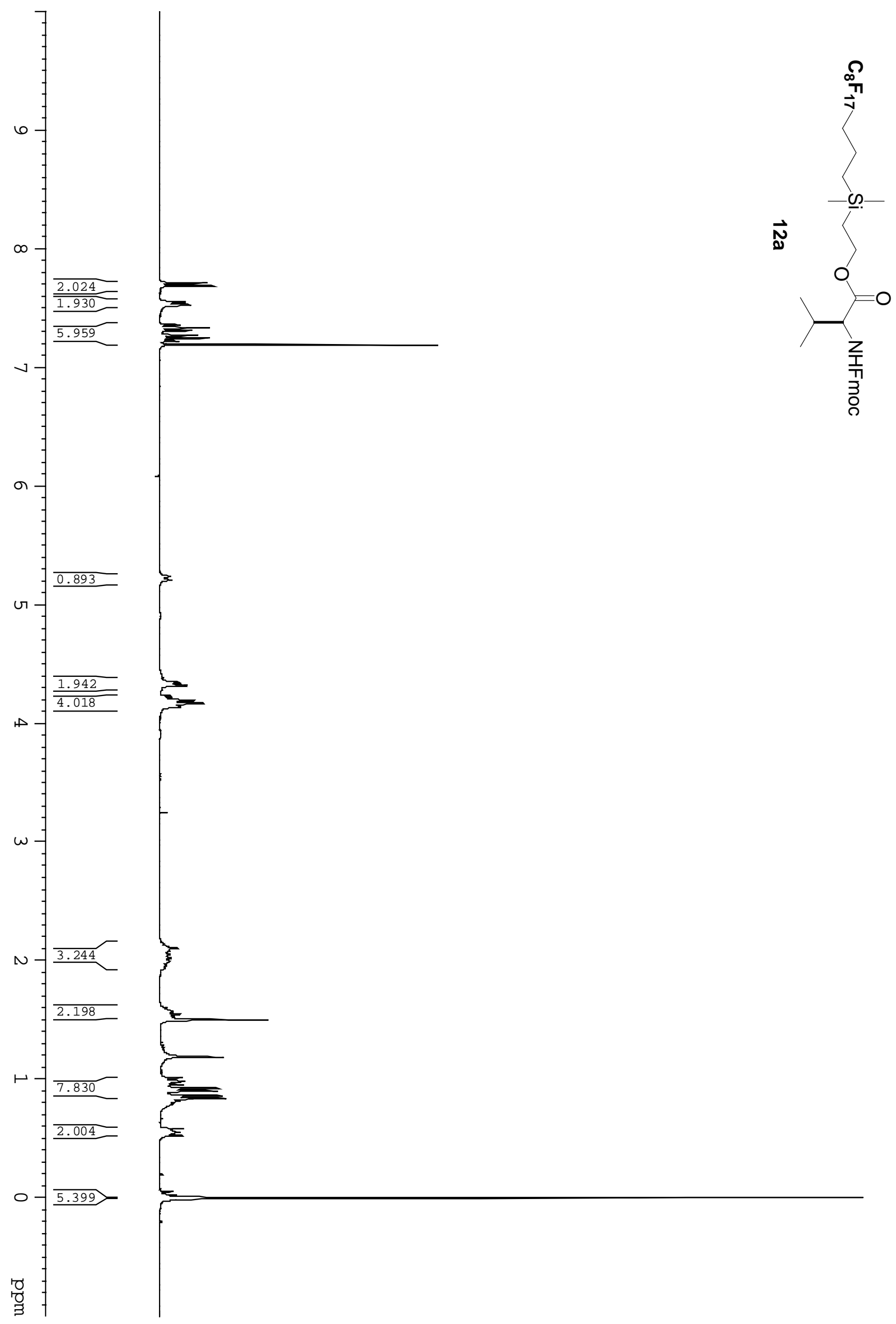




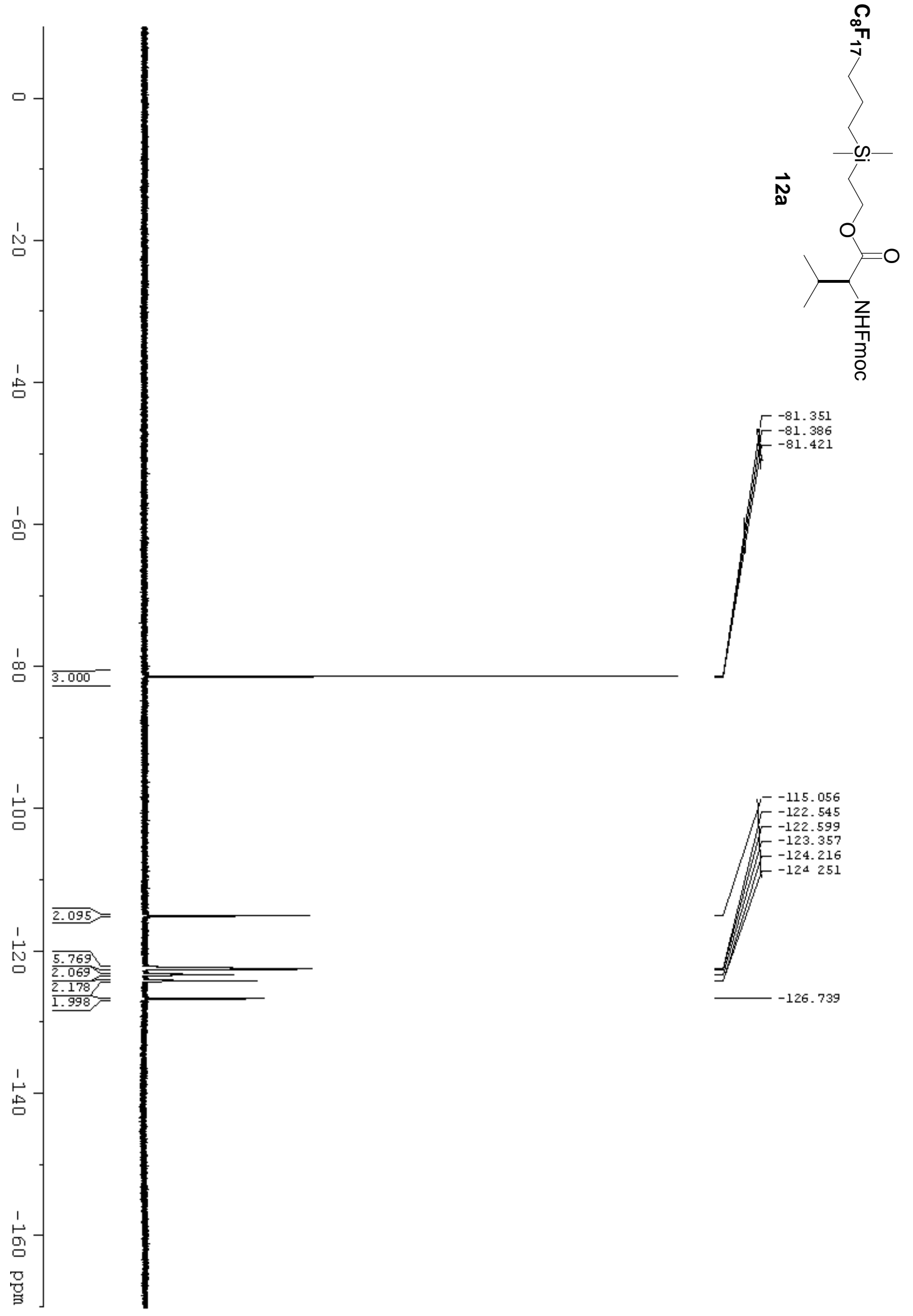



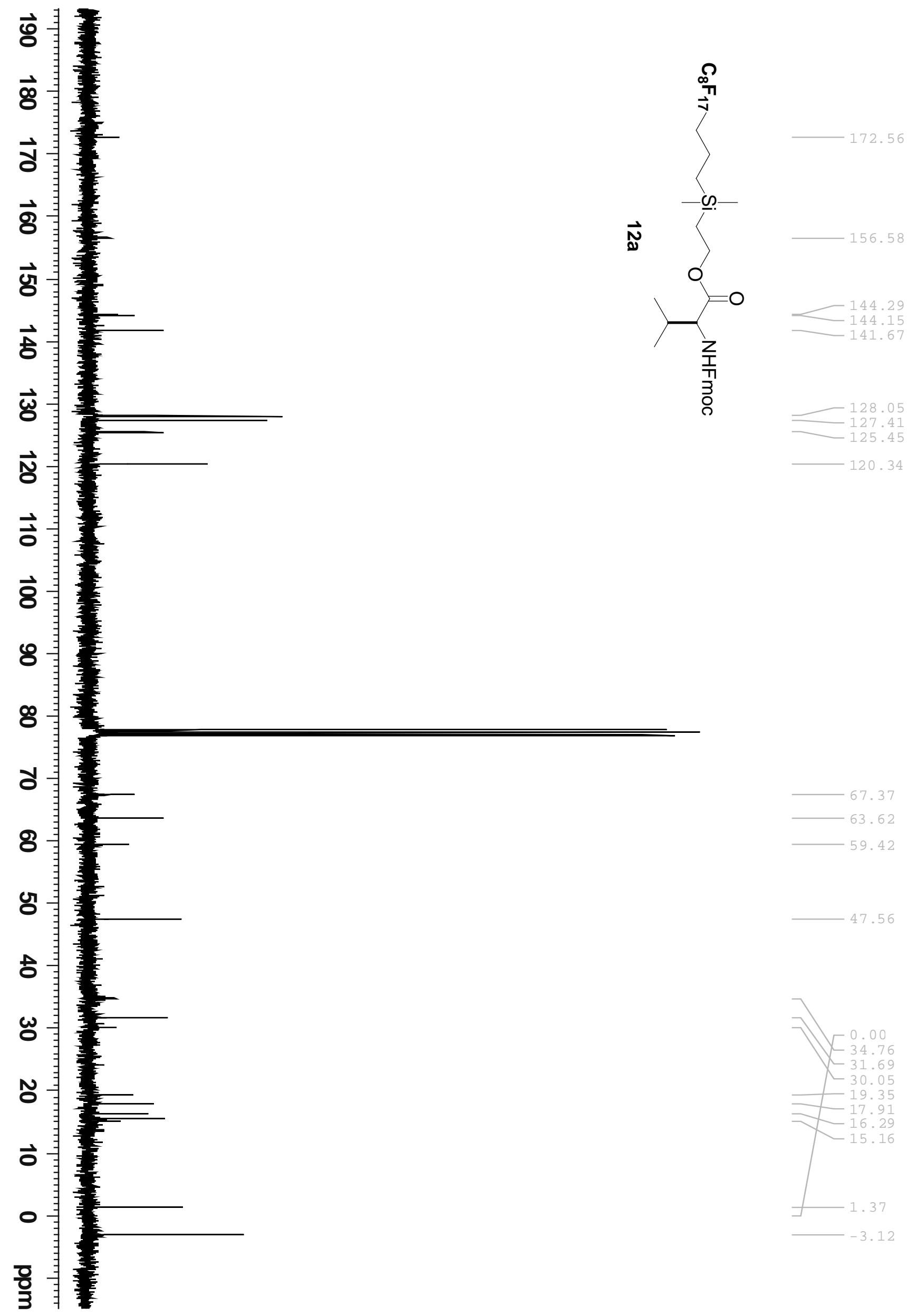

$\begin{array}{r}128.05 \\ -\quad 127.41 \\ \hline\end{array}$ $-125.45$
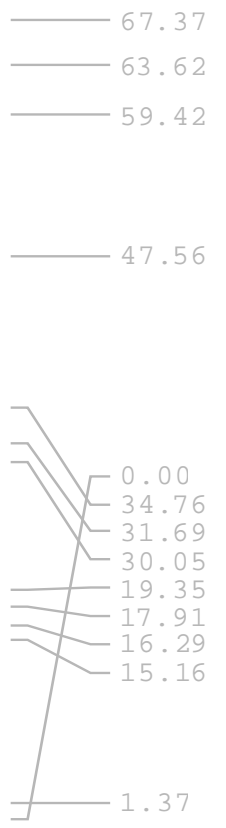


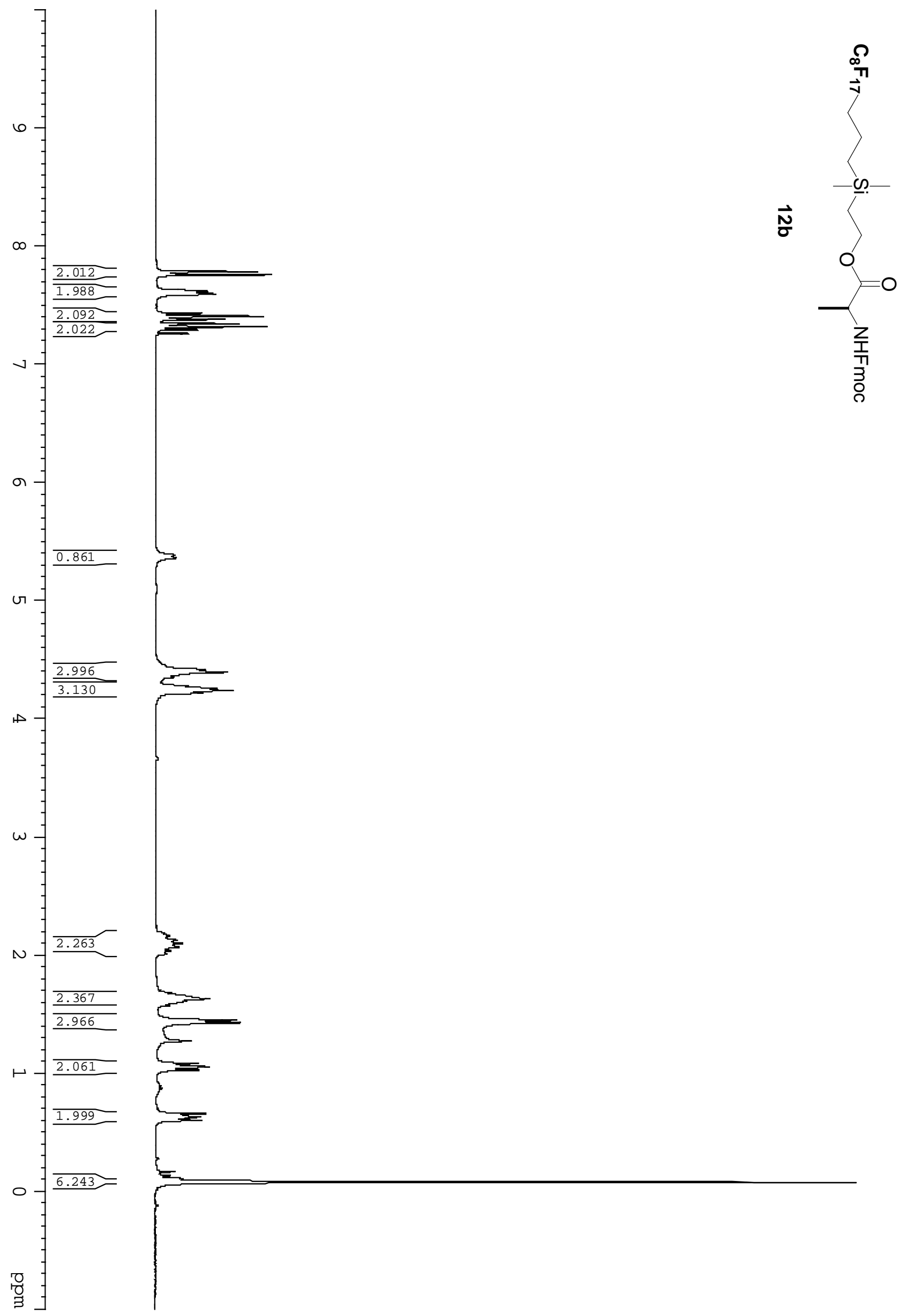




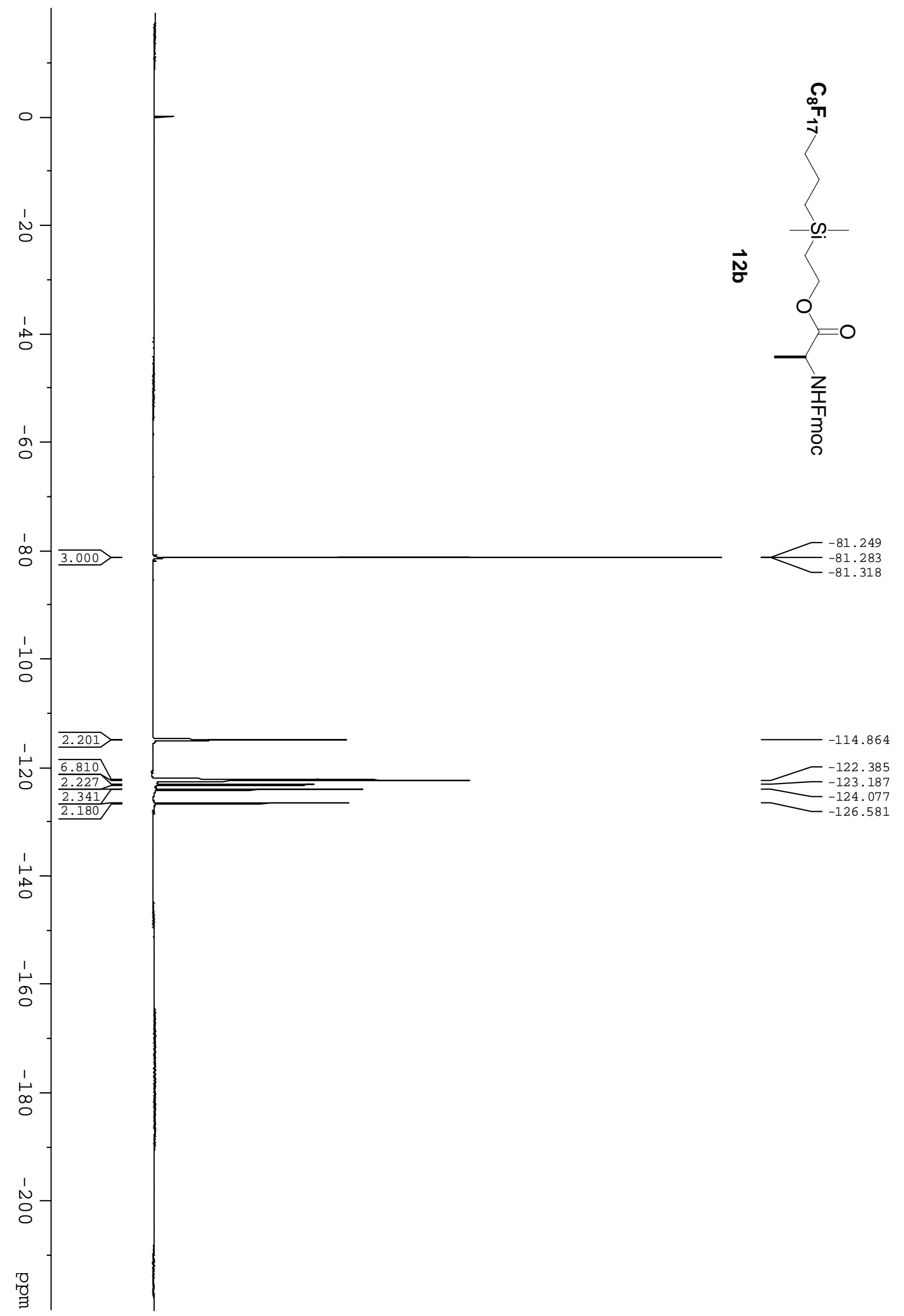



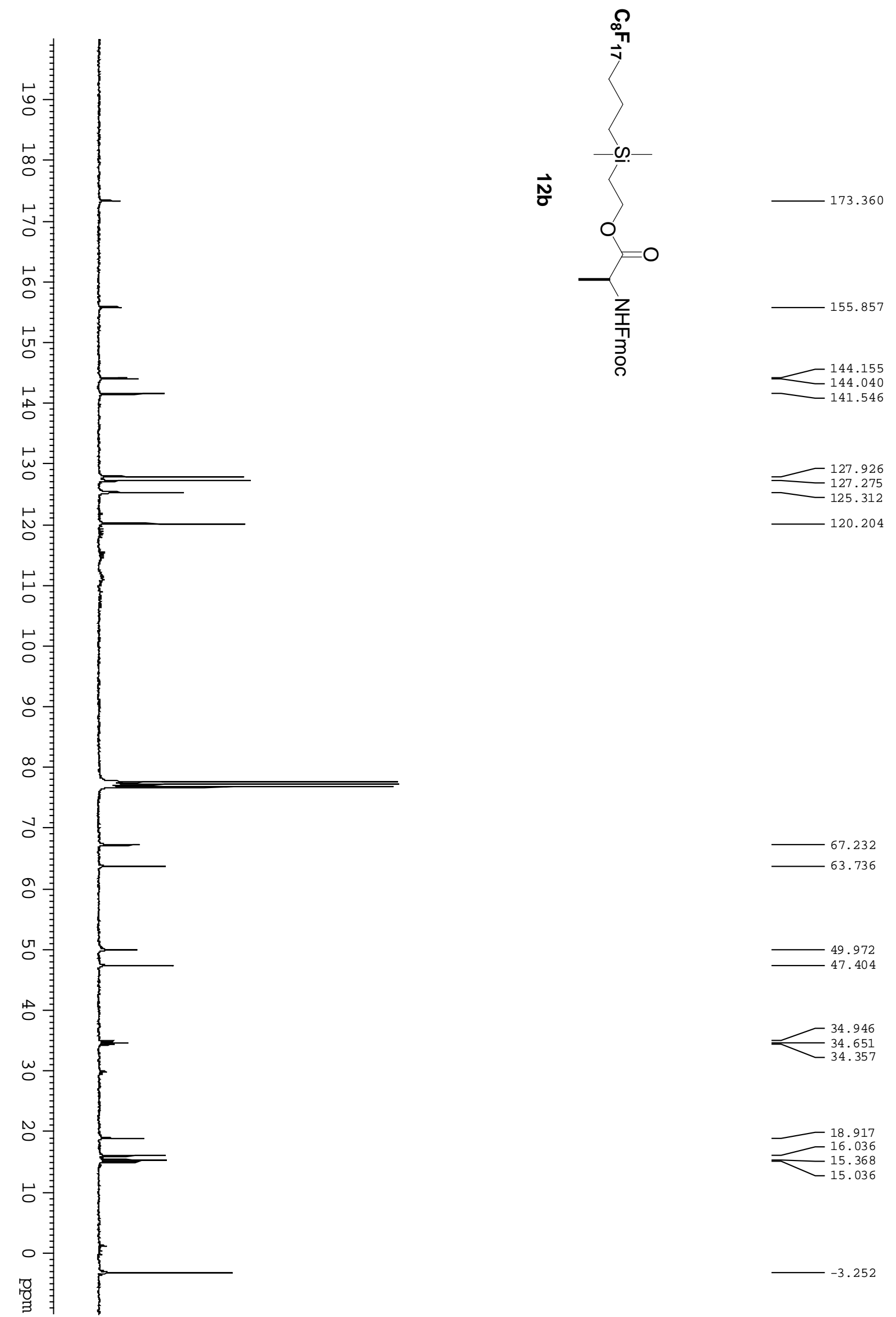


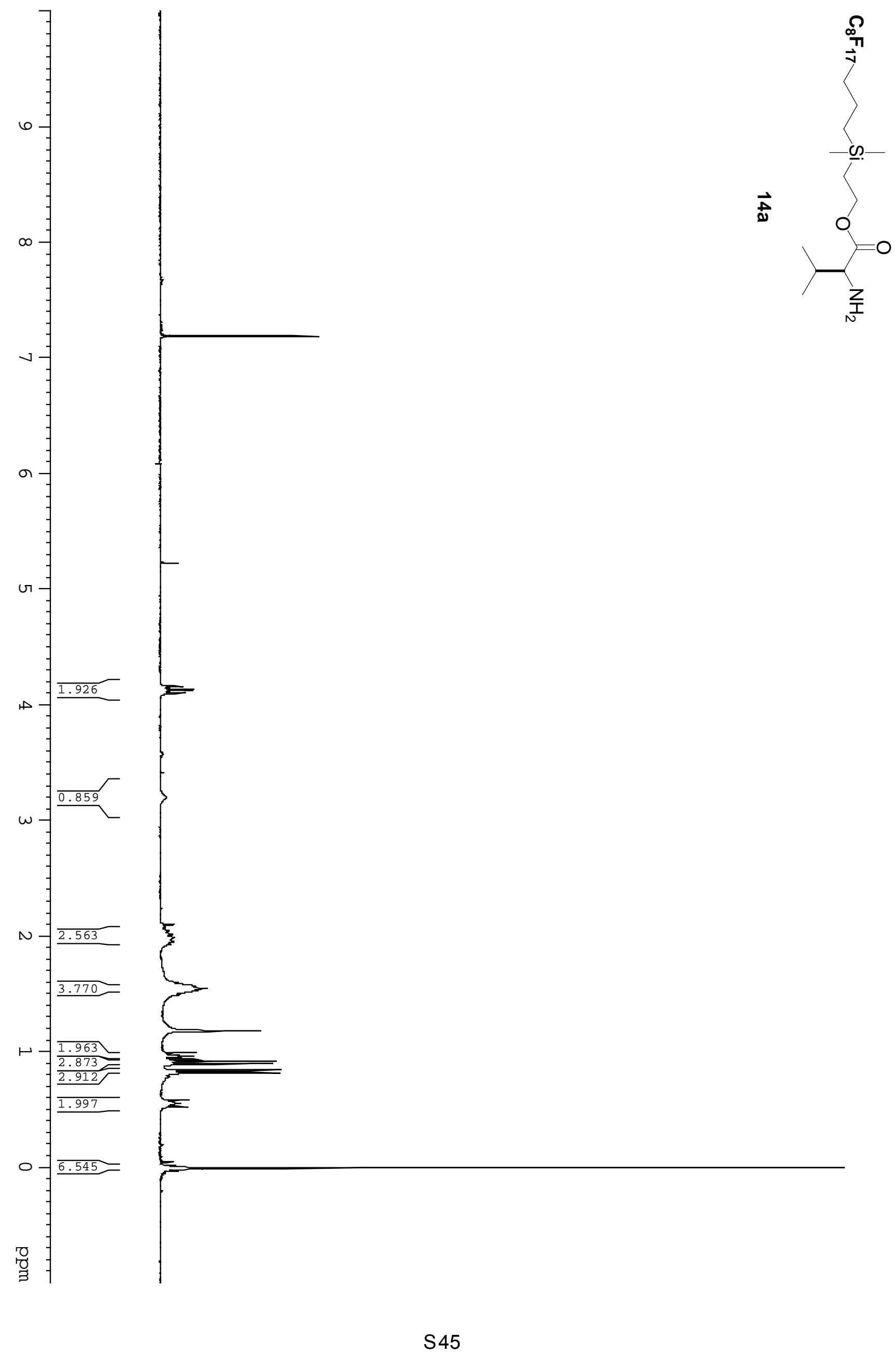




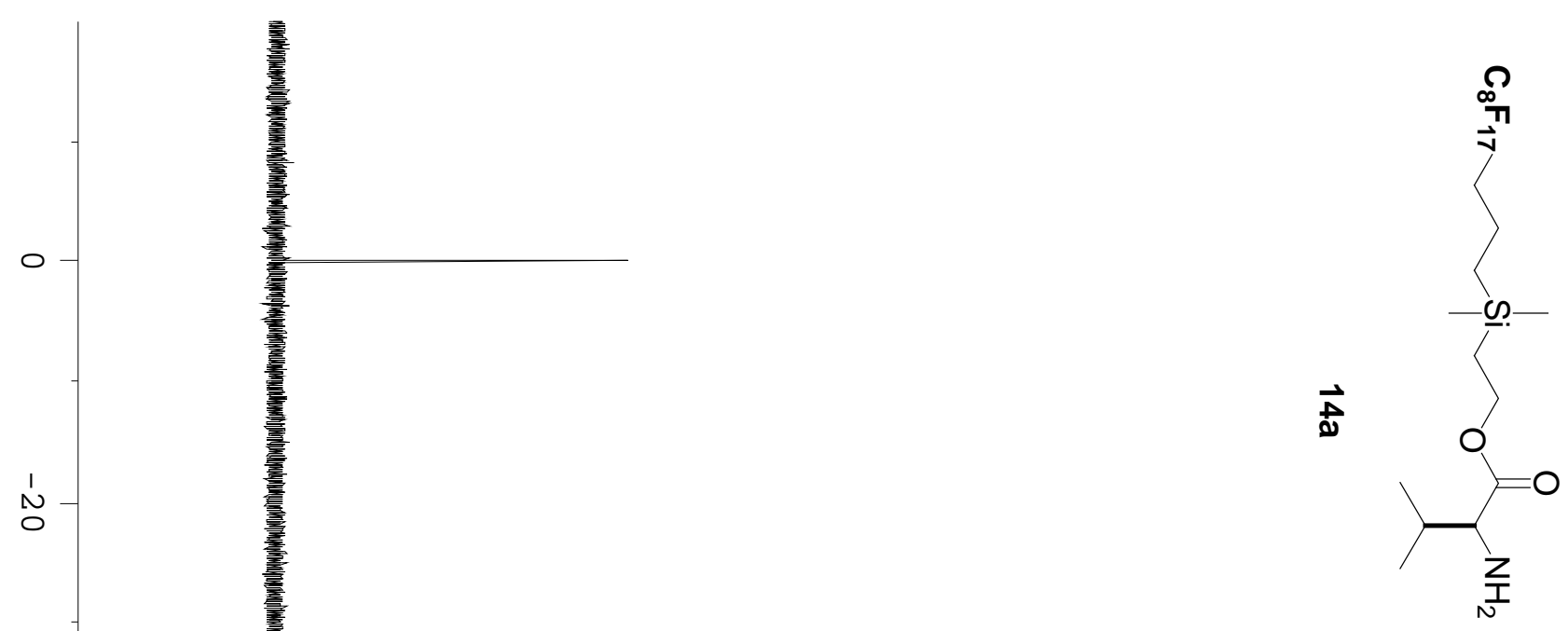

字

$\stackrel{1}{\infty}-3.028$

$-81.202$

$-81.237$

$-81.272$

$\stackrel{\leftrightarrow}{\odot}$

ஓ่

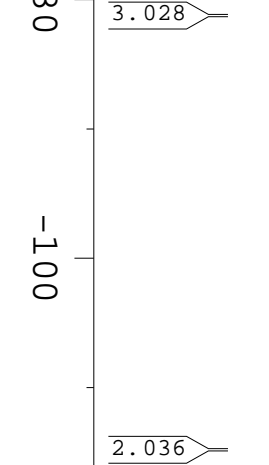

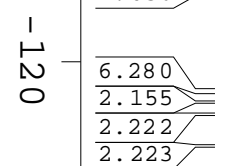
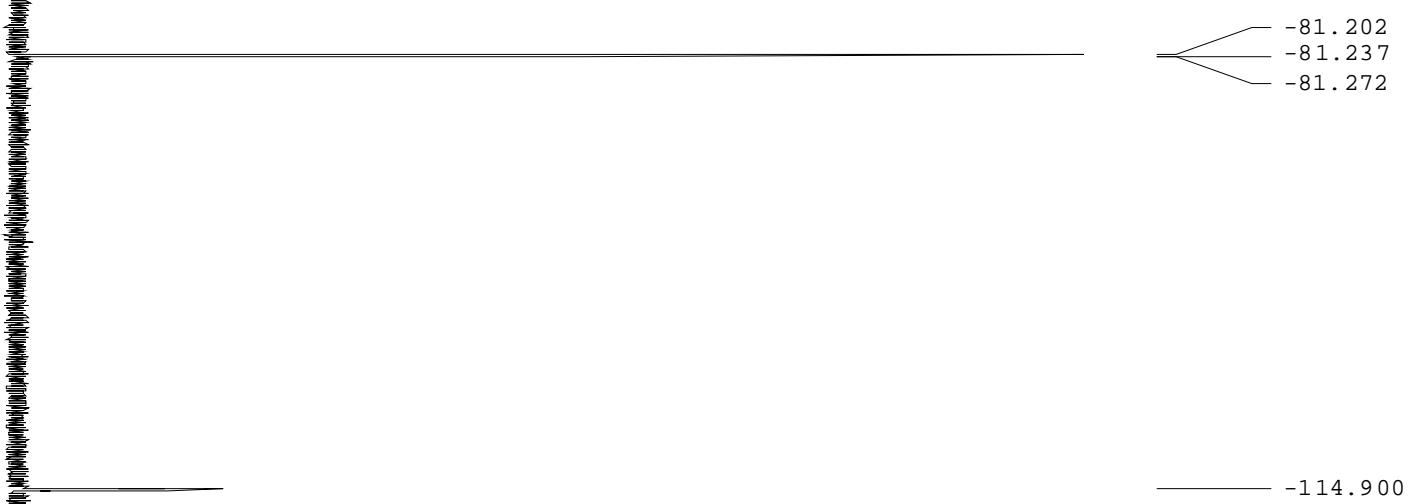

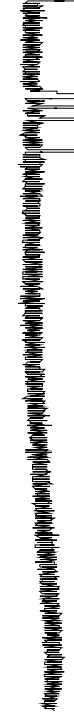

$-122.389$

$-123.206$

$-124.113$

$-126.586$

응 


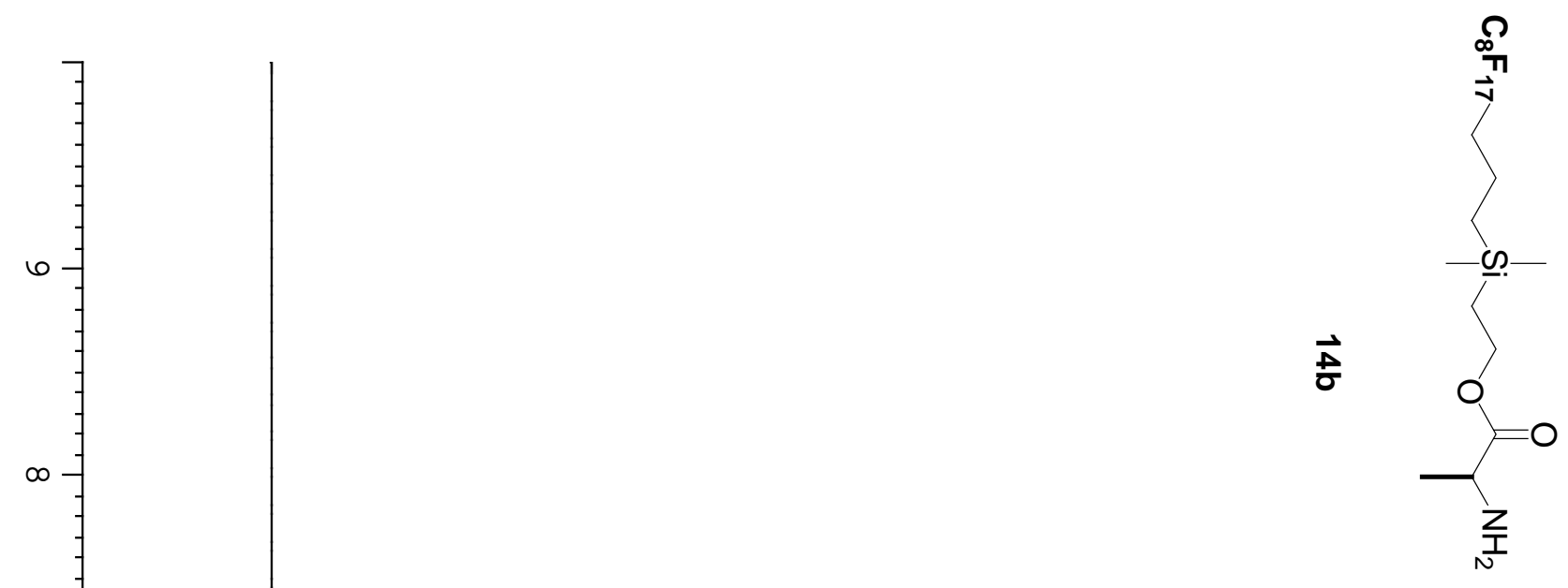




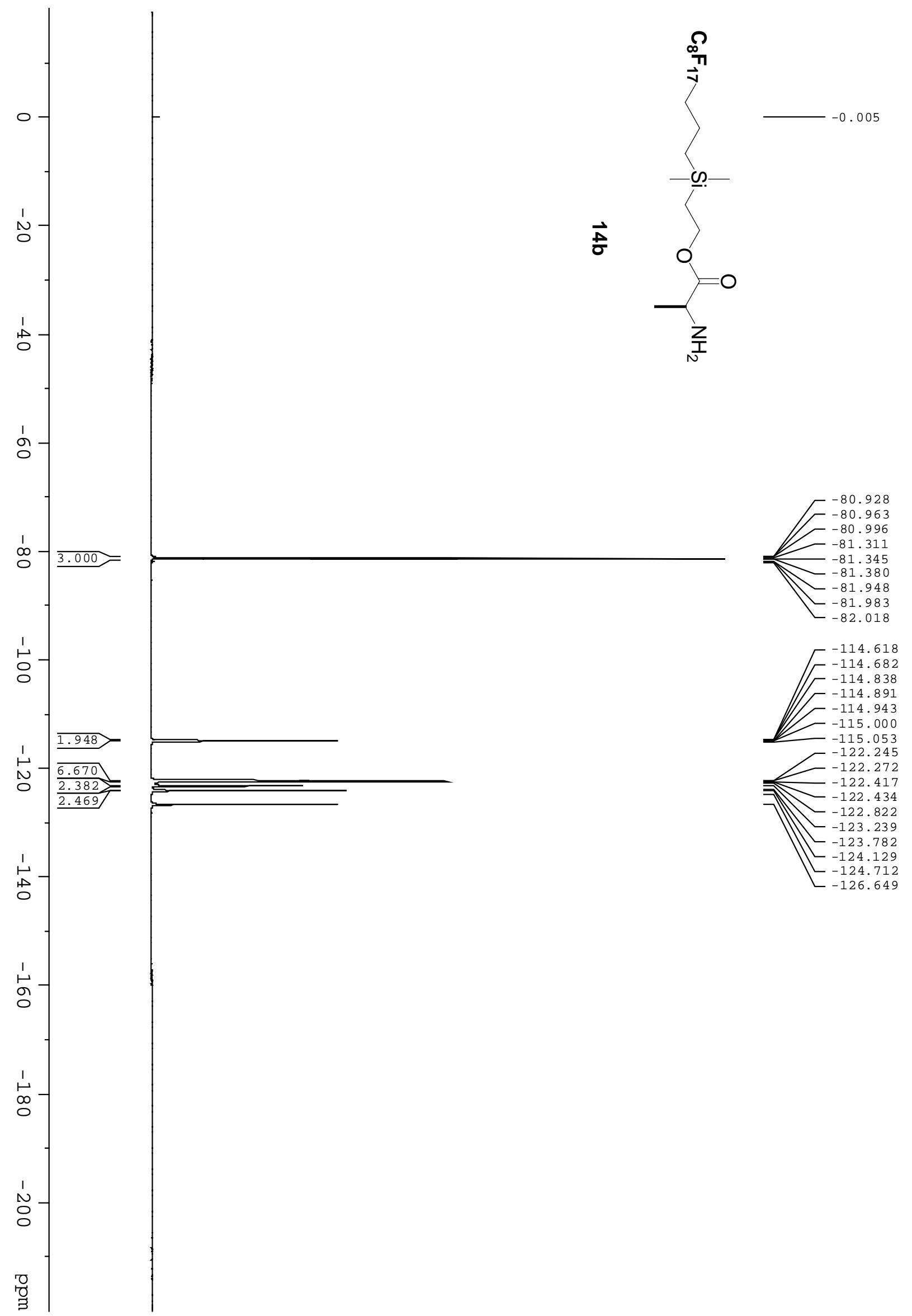




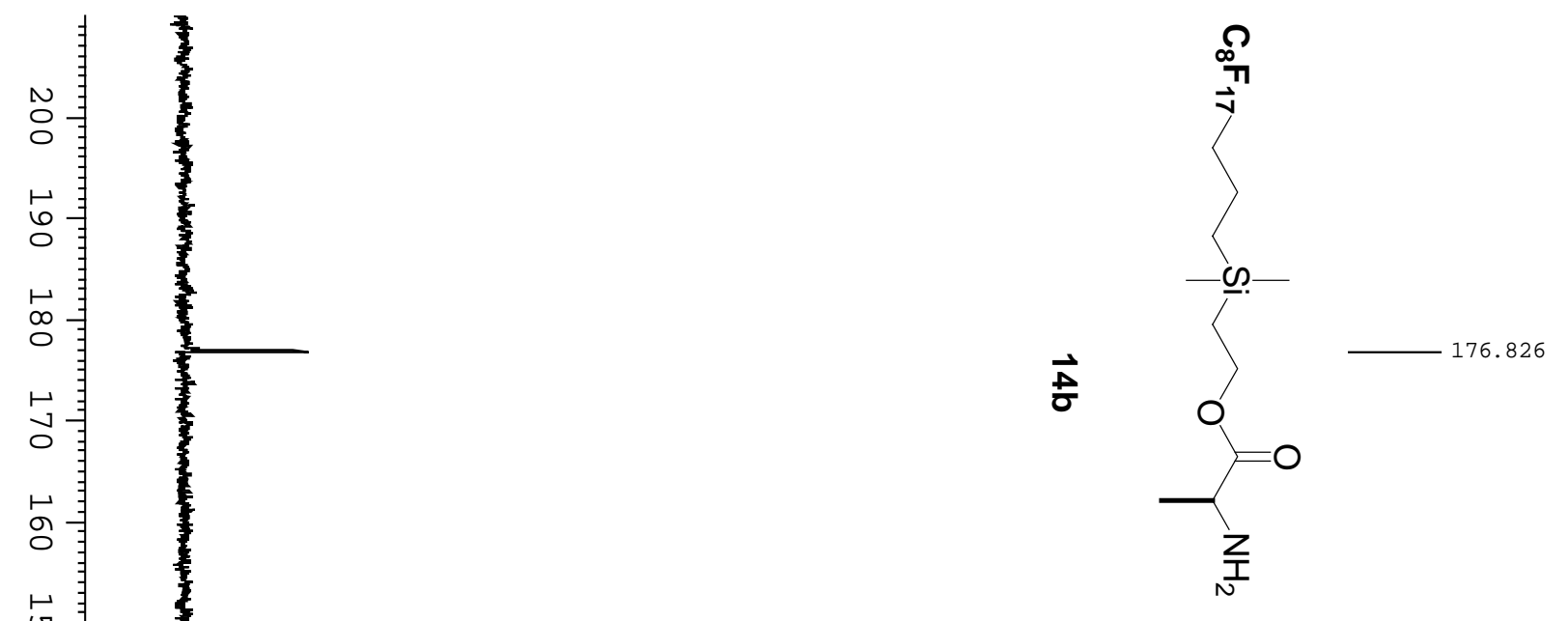

要

c.

N

占

$\circ$

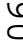

$\infty$

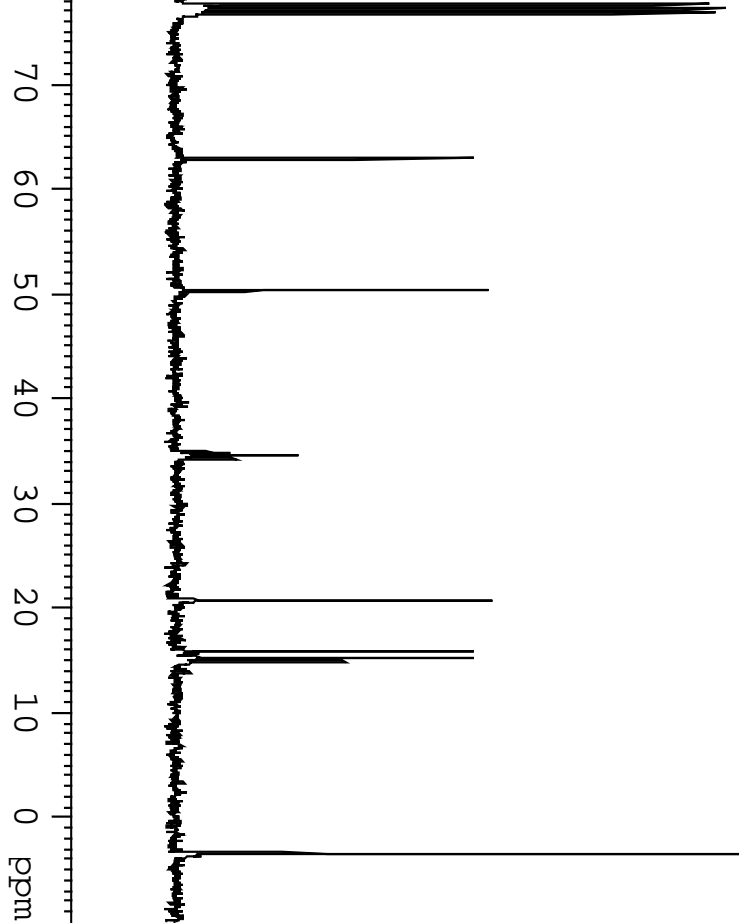

62.844

50.249 


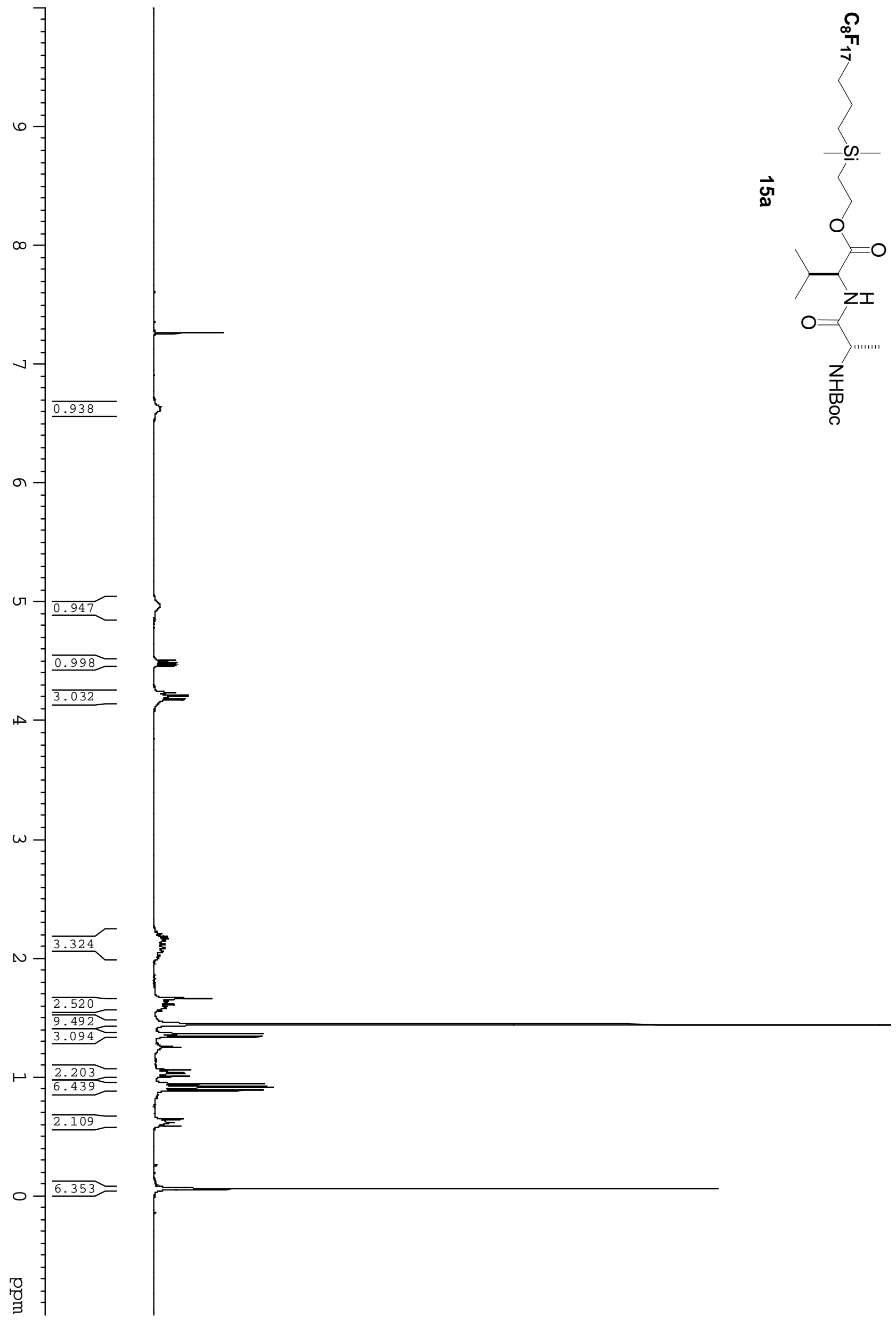




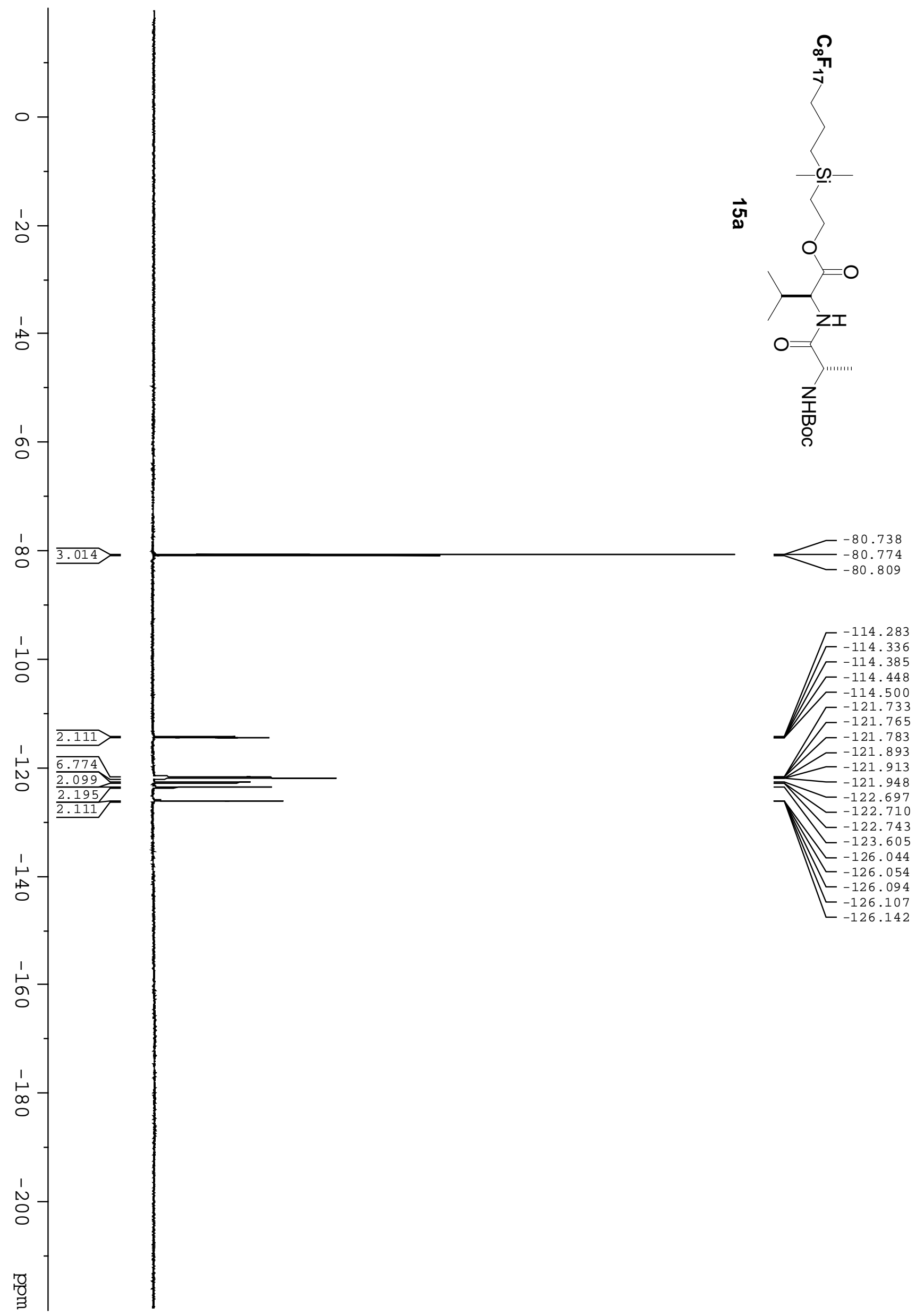




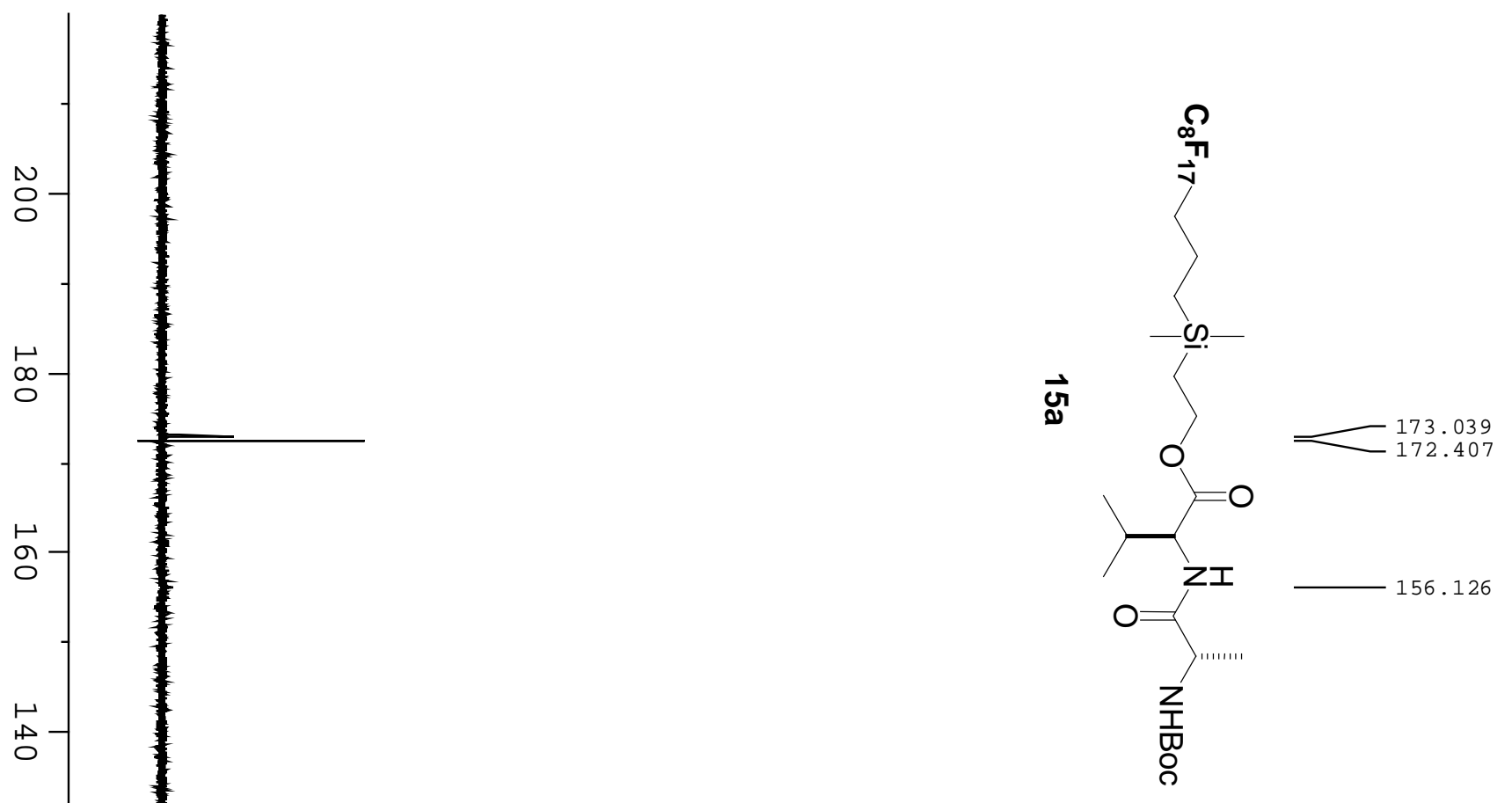

$\stackrel{\Perp}{\circ}$

$\stackrel{\leftrightarrow}{8}$

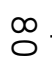

8

63.776

57.675

50.643

$\stackrel{+}{\circ}$

35.303

35.011

34.715

28.867

$-19.558$

18.130

$-16.504$

15.709

$-15.445$

$-15.396$

$-15.339$

$-2.899$

몰

! 


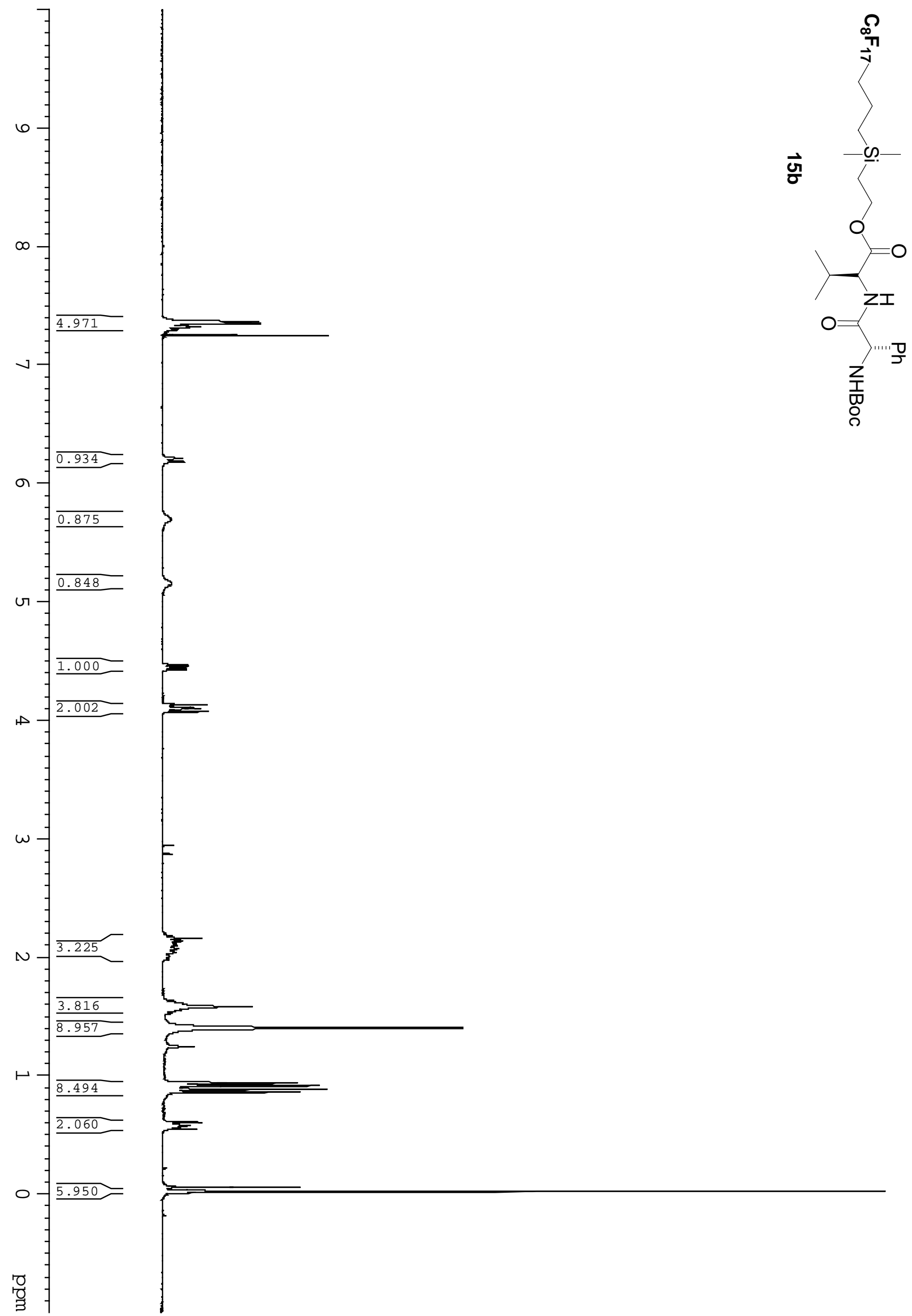




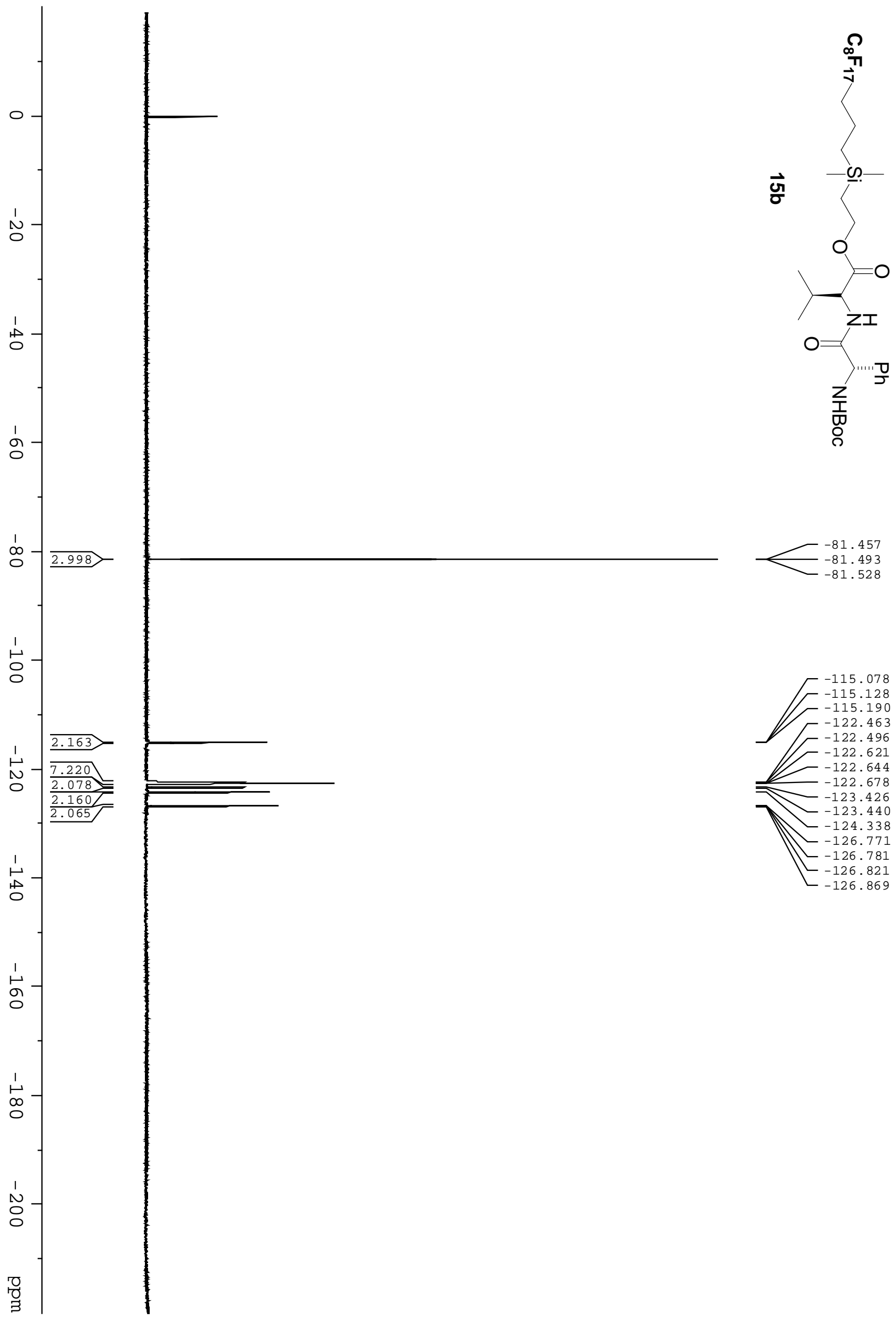




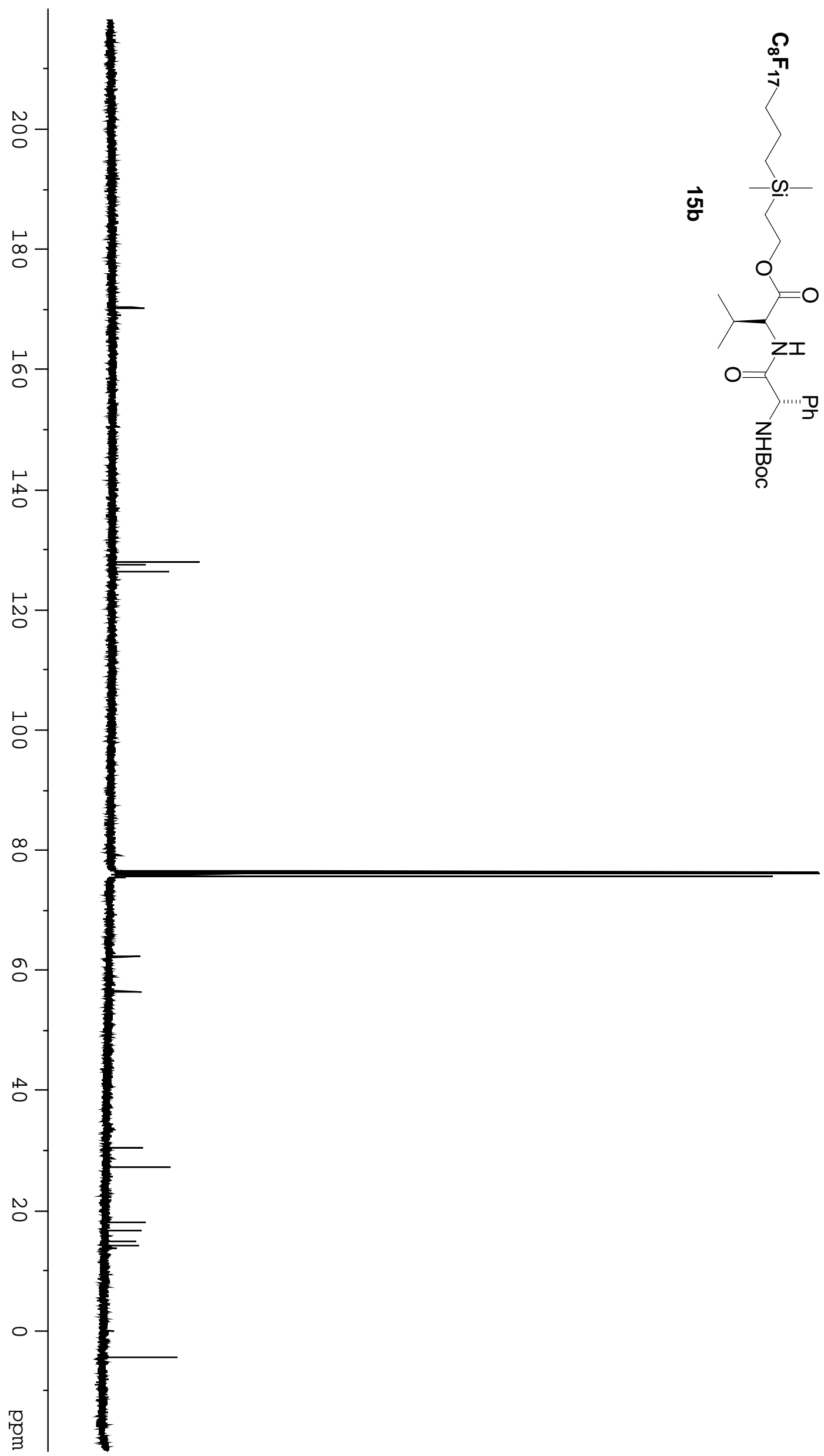

170.276 169.018

136.915

128.004 128.004 126.238 79.167

33.688

33.395

33.102

30.352

27.249

17.928

16.666

14.827

14.075

$-13.775$

$-4.525$ 


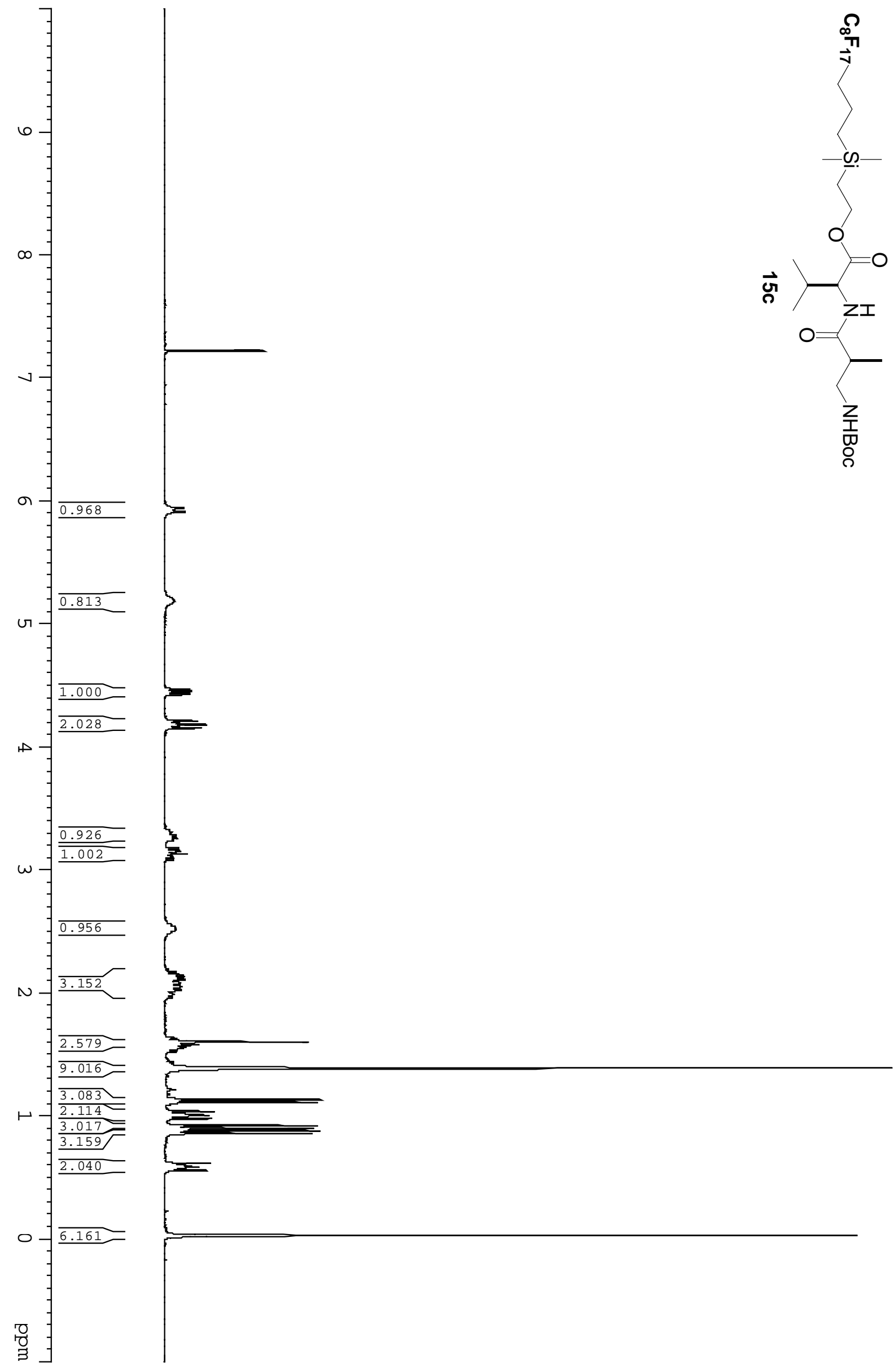



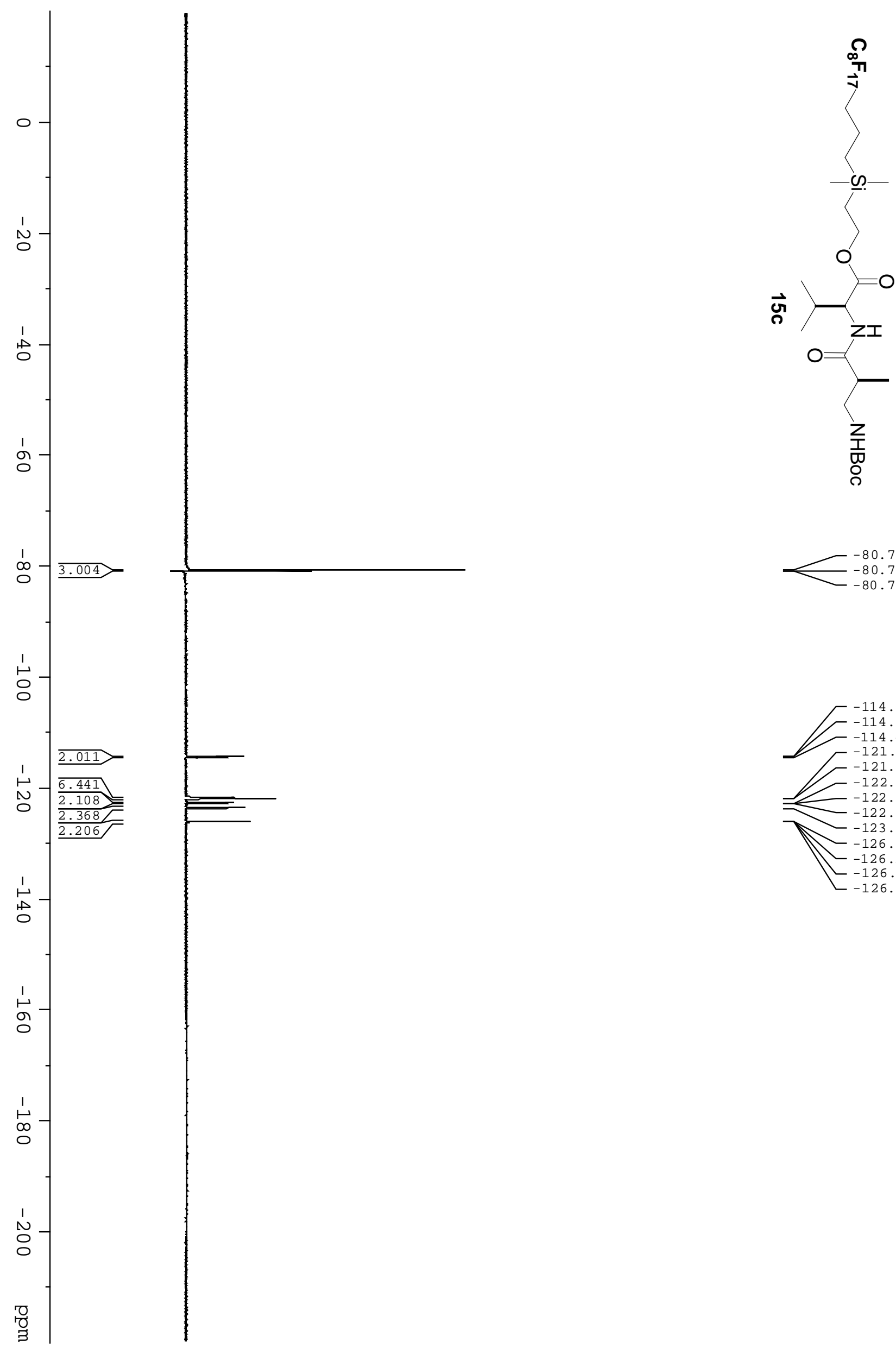

80.724

$-80.759$

$-80.794$

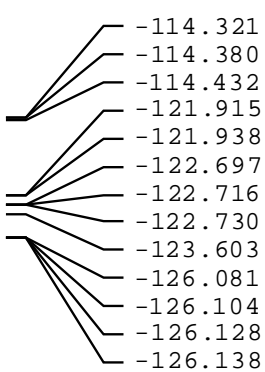




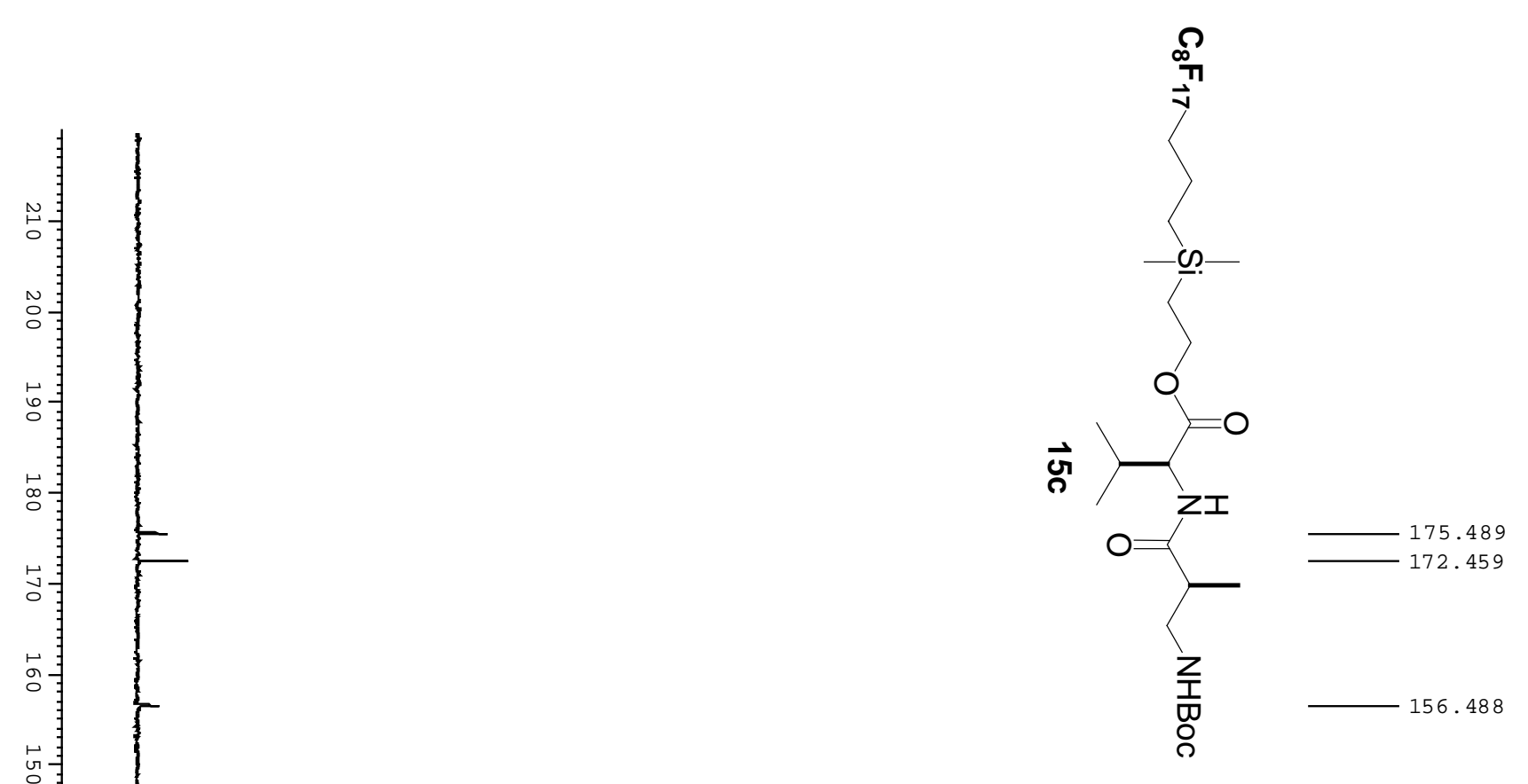

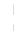

$\stackrel{\vec{\omega}}{\circ}$

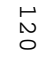

㟔

$\stackrel{\circ}{8}$

8

$\stackrel{\omega}{\odot}$

E

$\stackrel{+}{\circ}$

$\omega$

긍겨

.

$\odot$

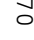

8

。

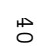

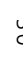

พ

$\bullet$

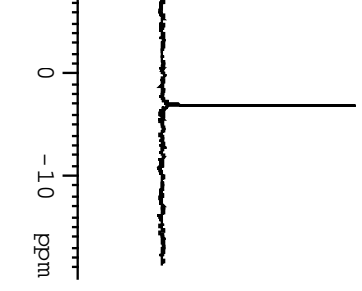

$-3.123$ 


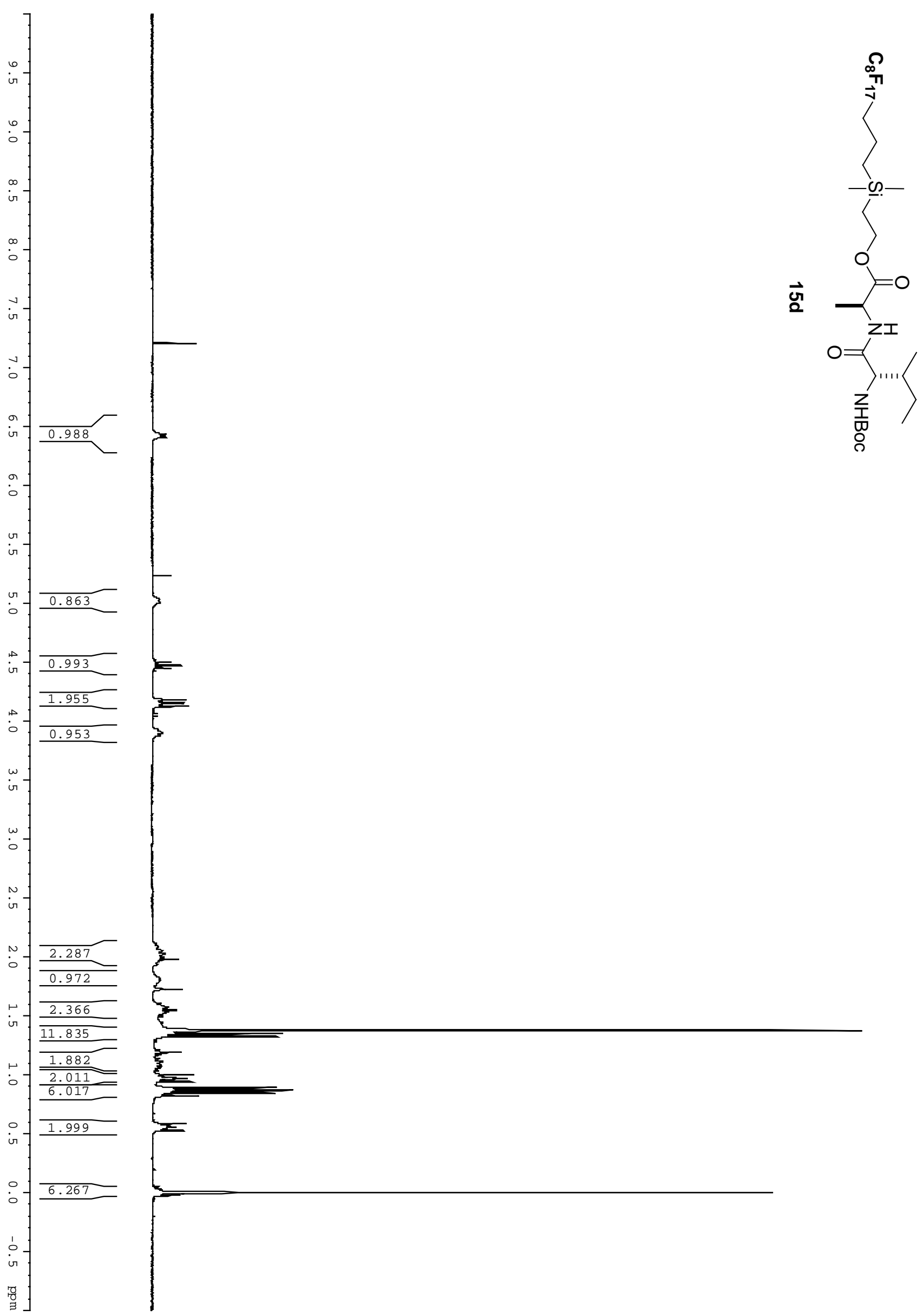



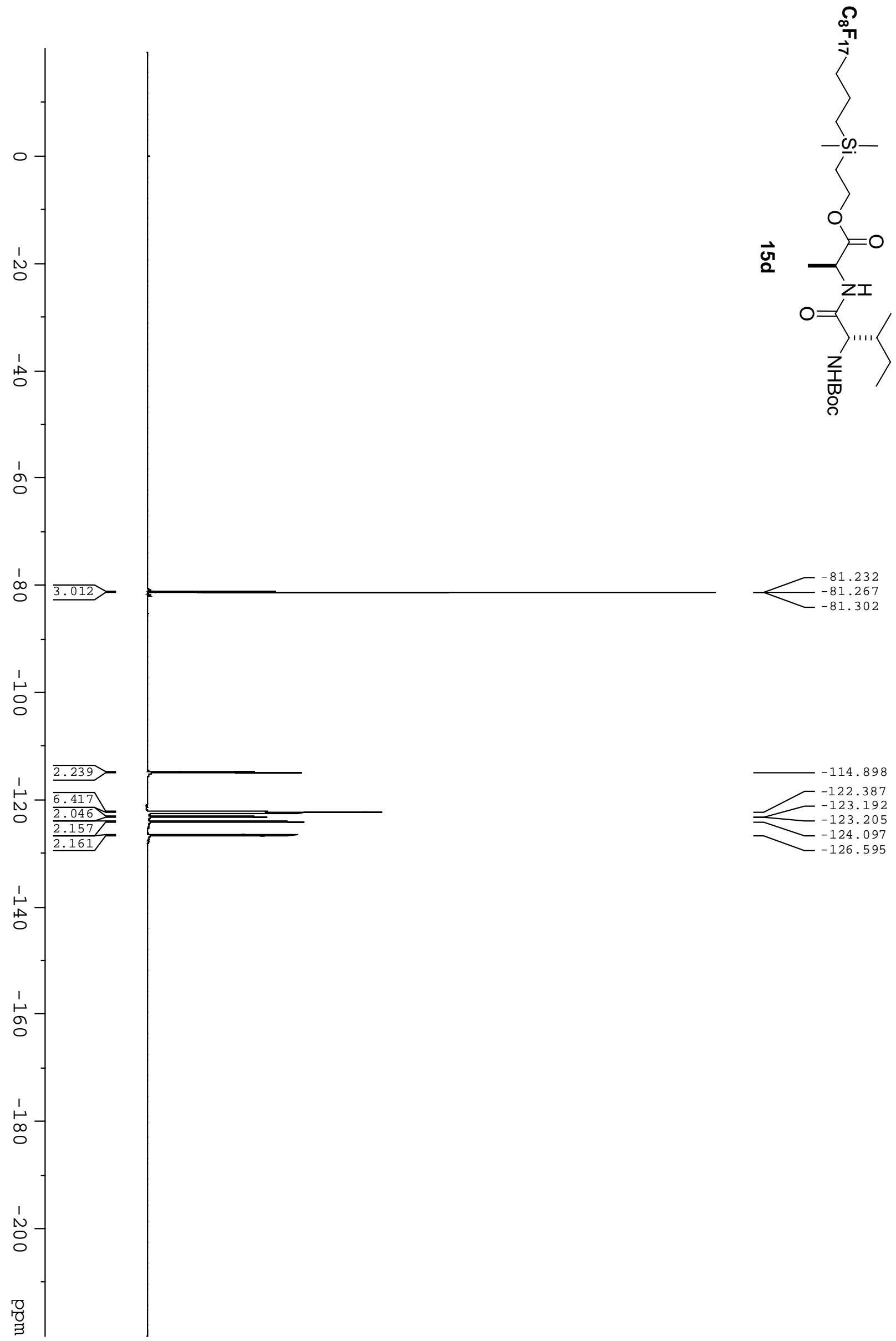

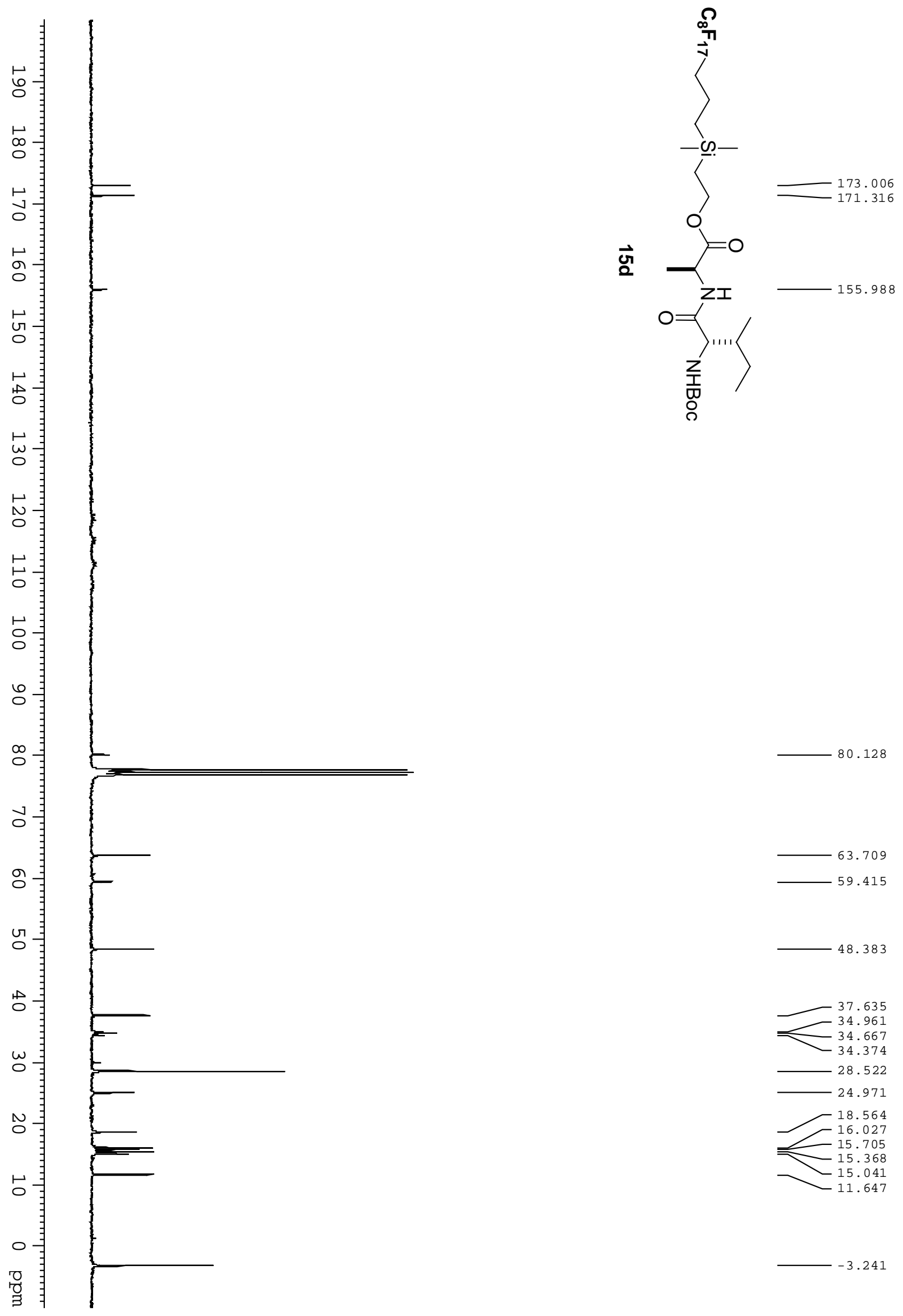

80.128

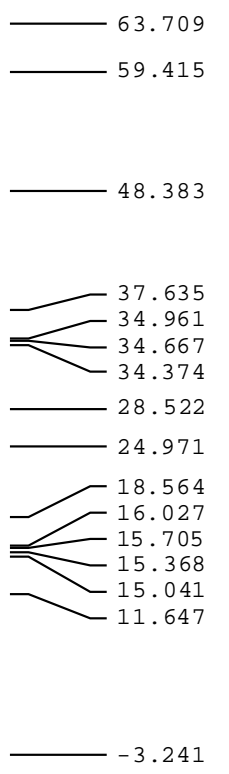




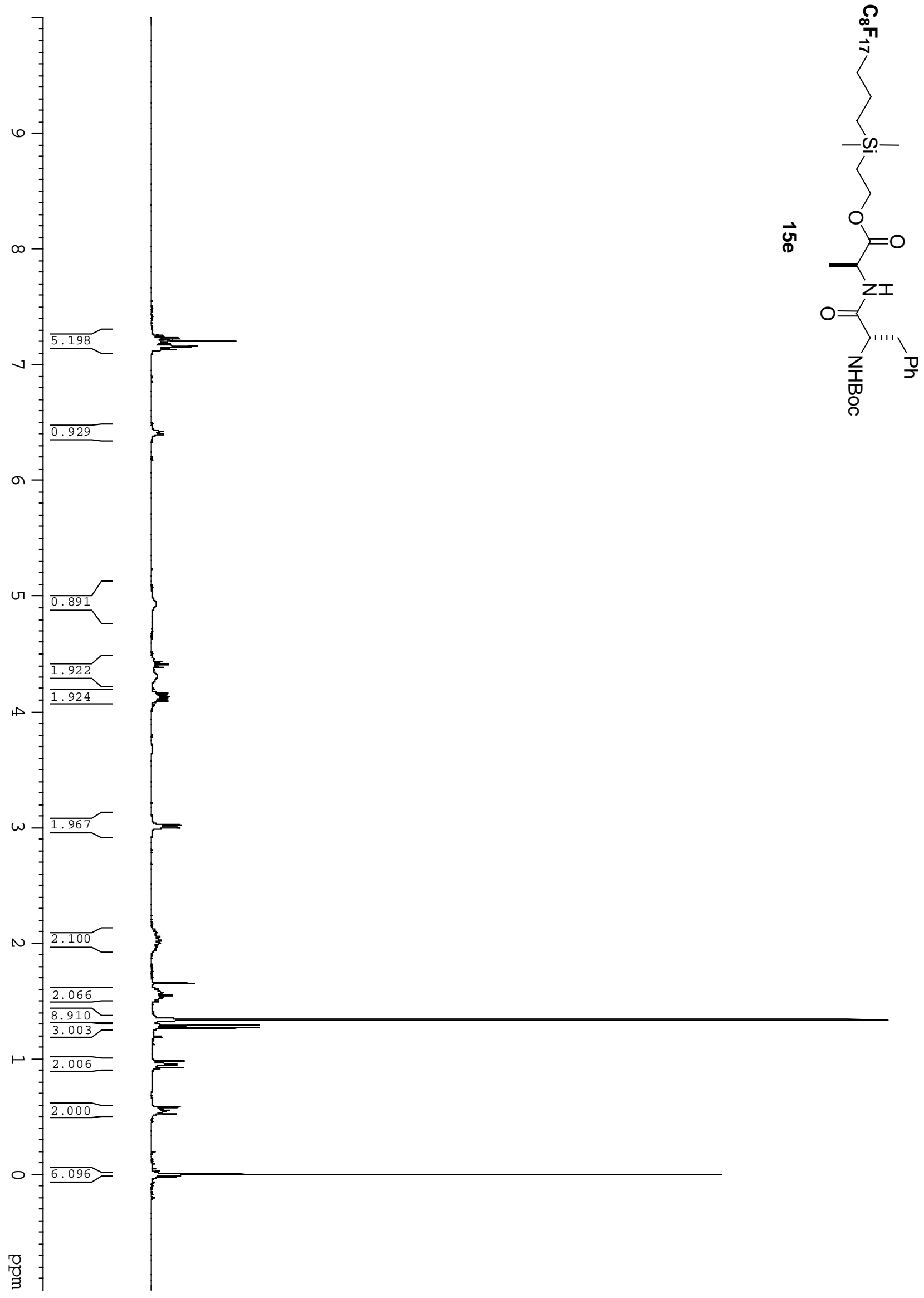




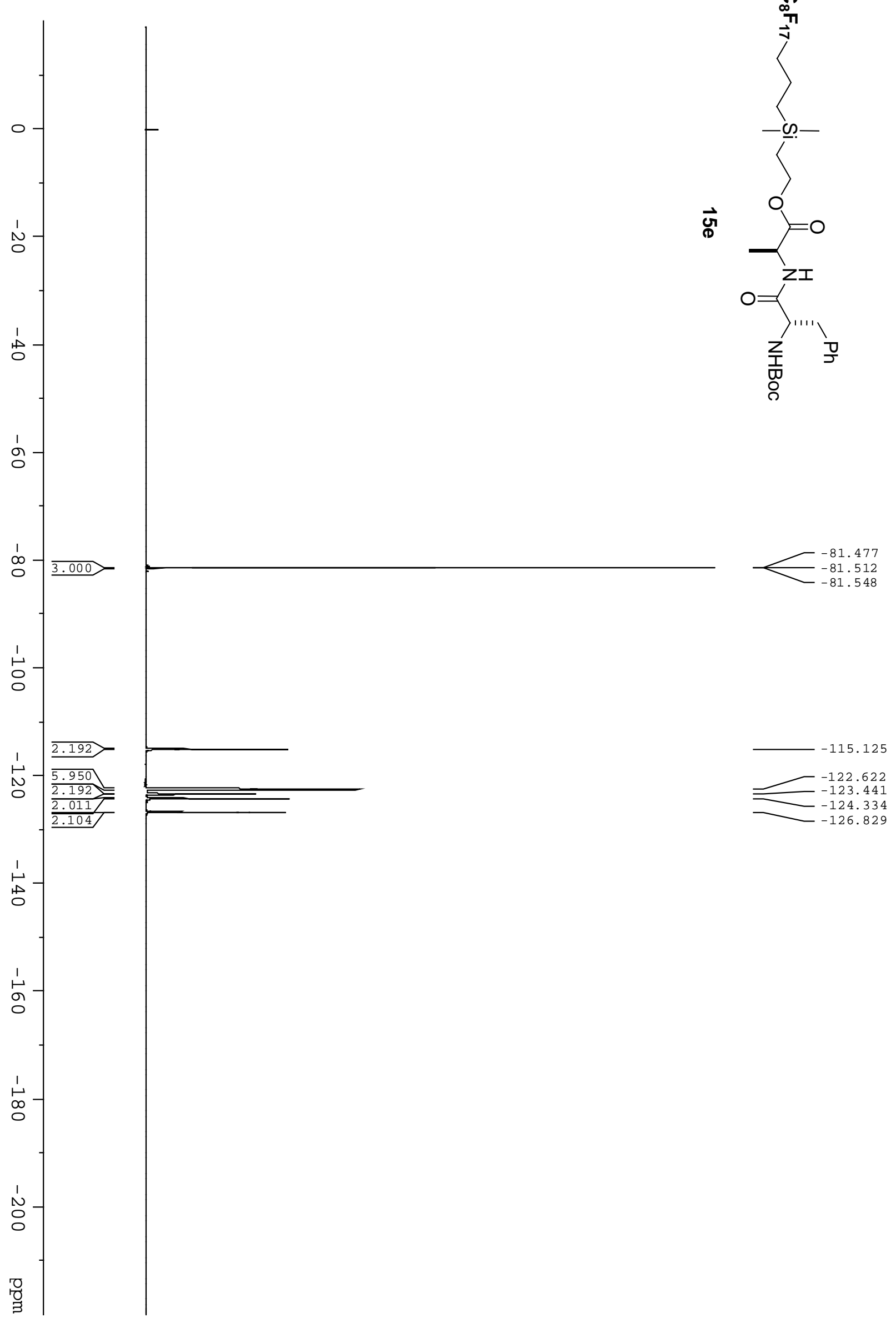



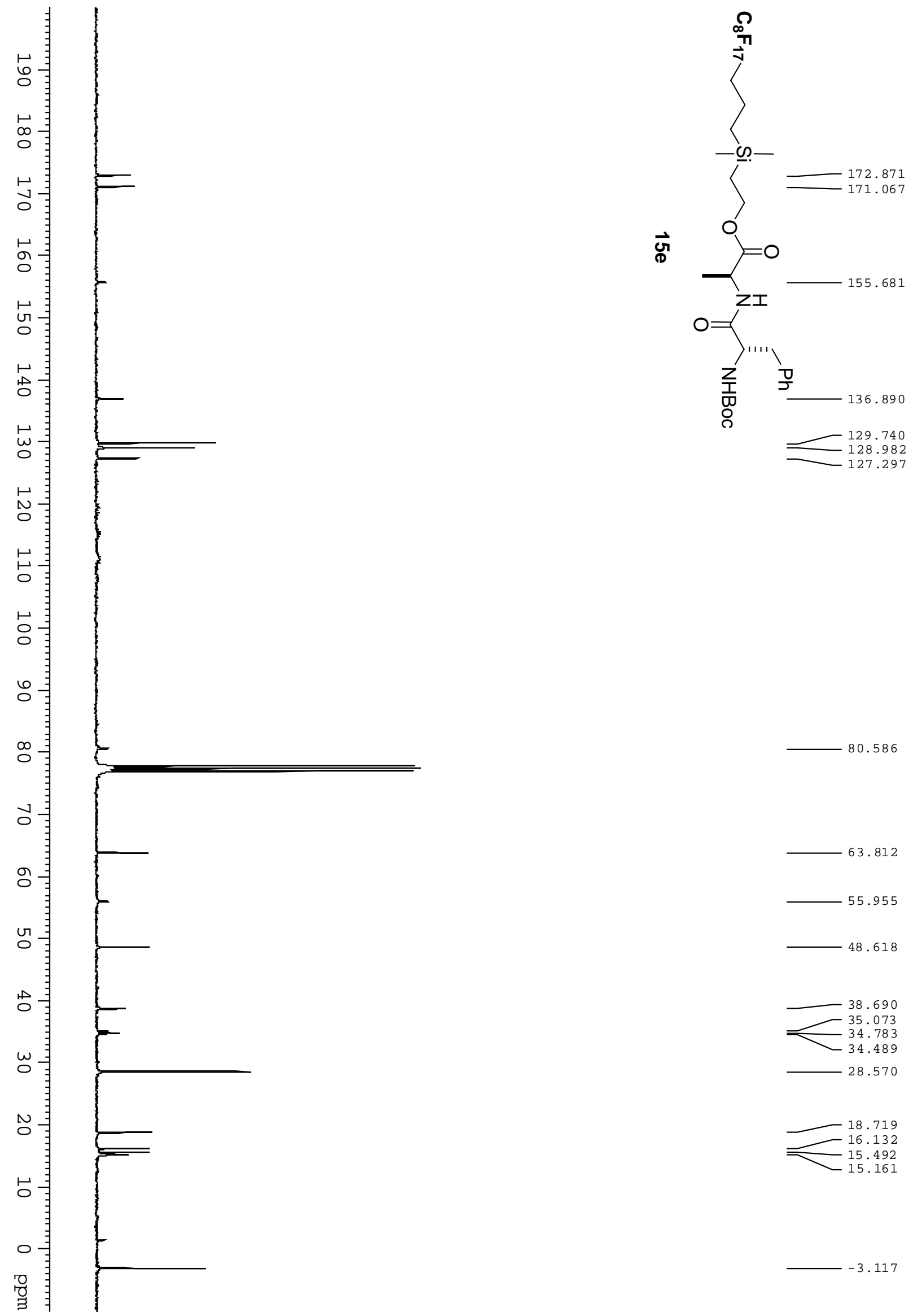

$-3.117$ 


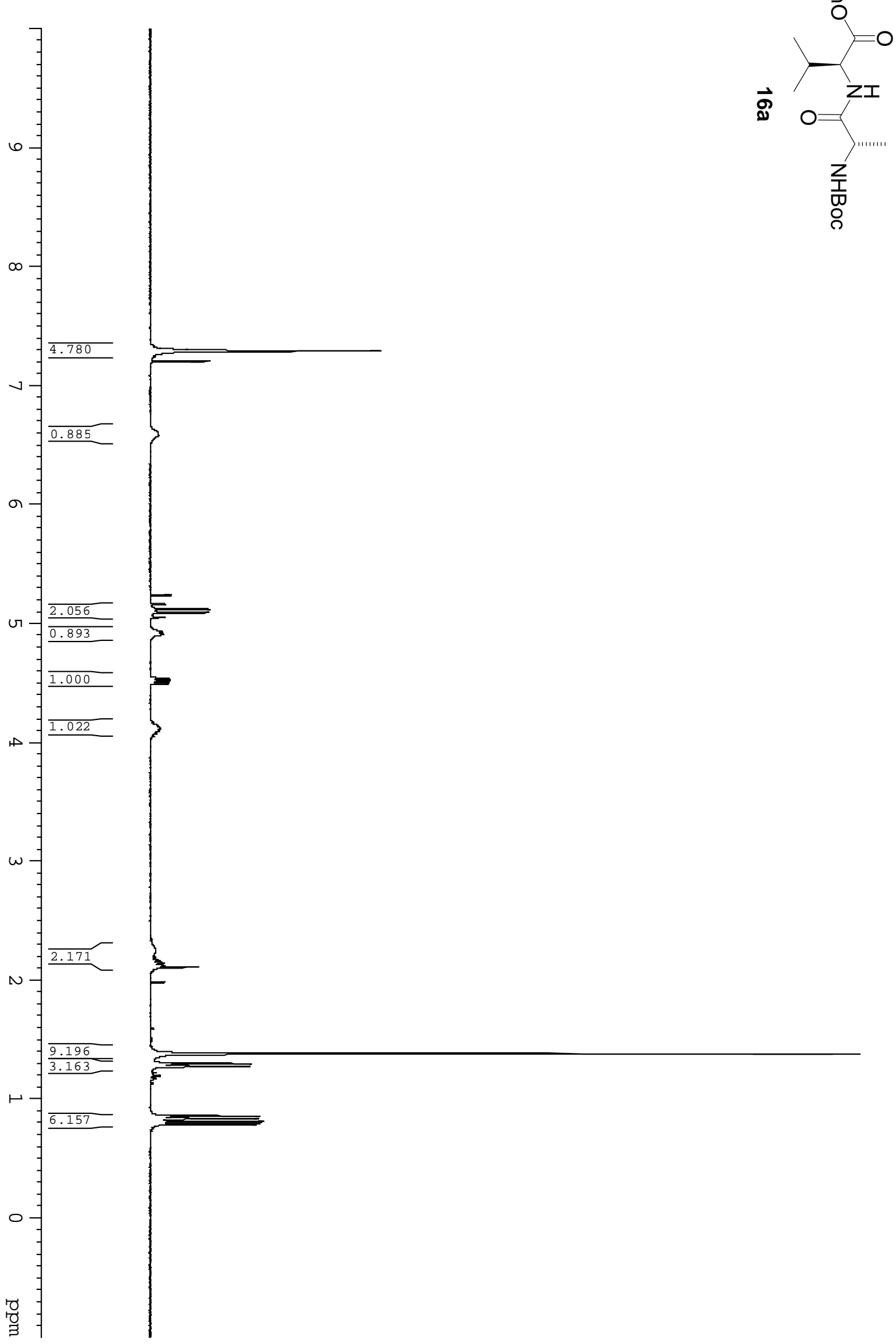




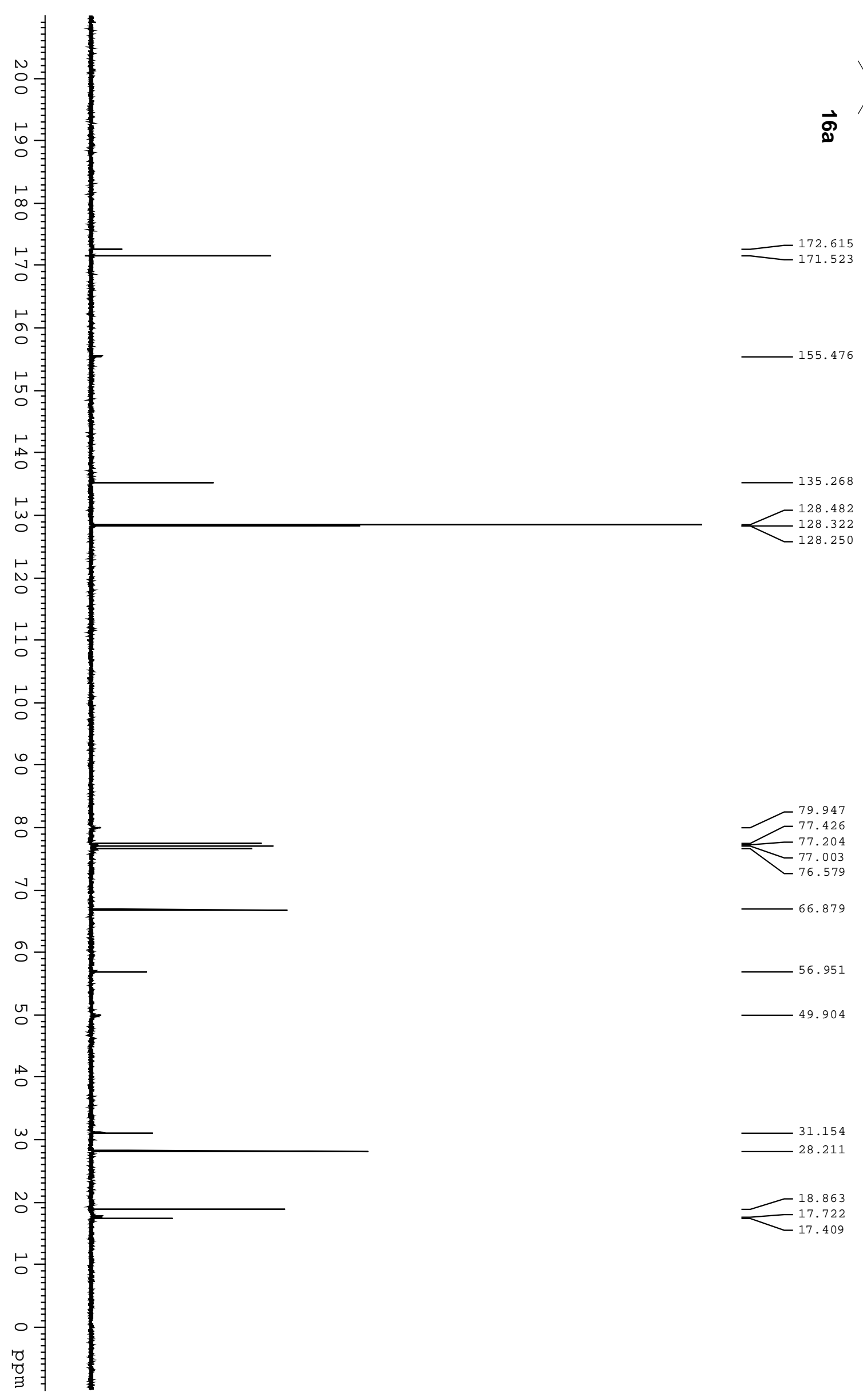




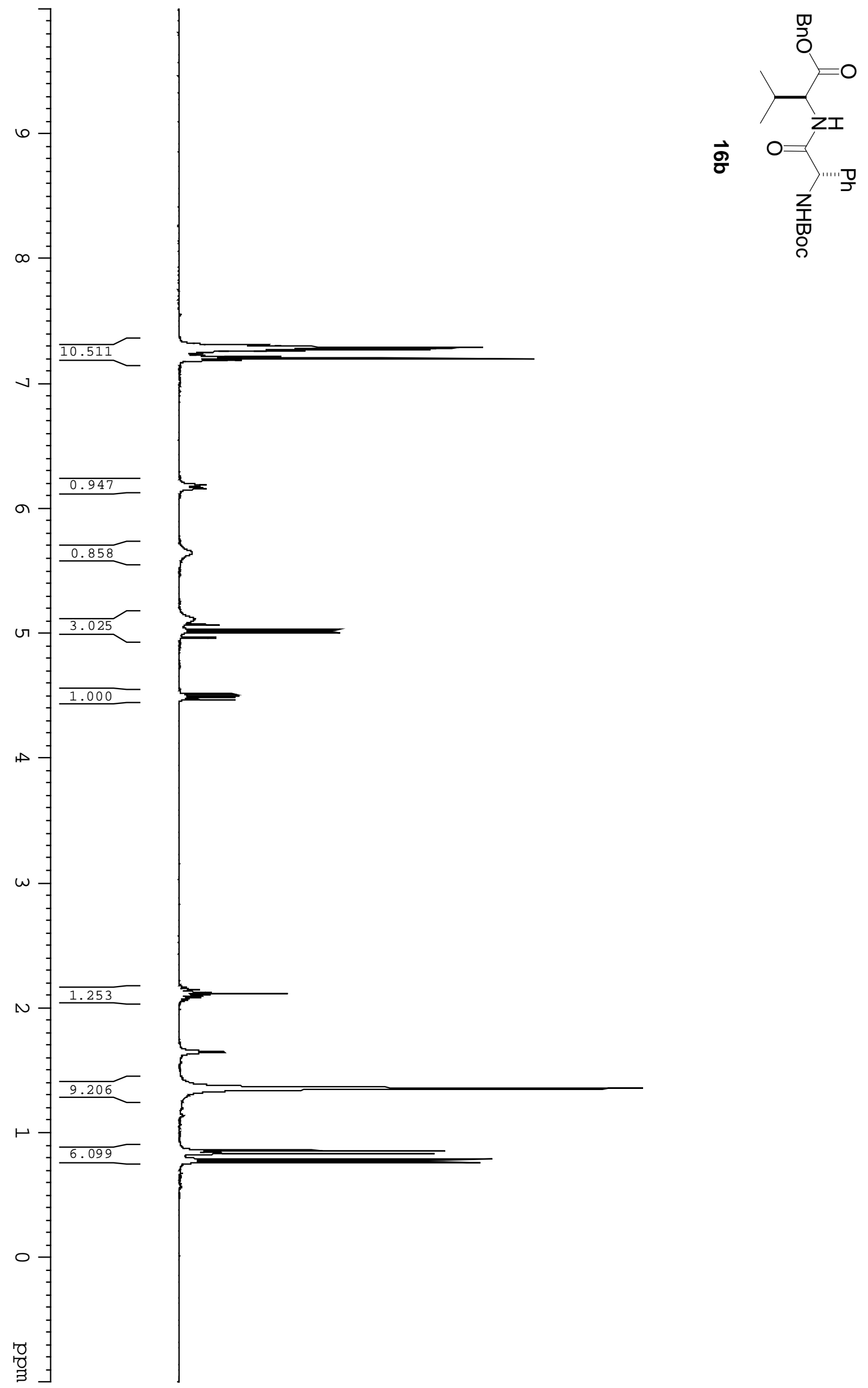



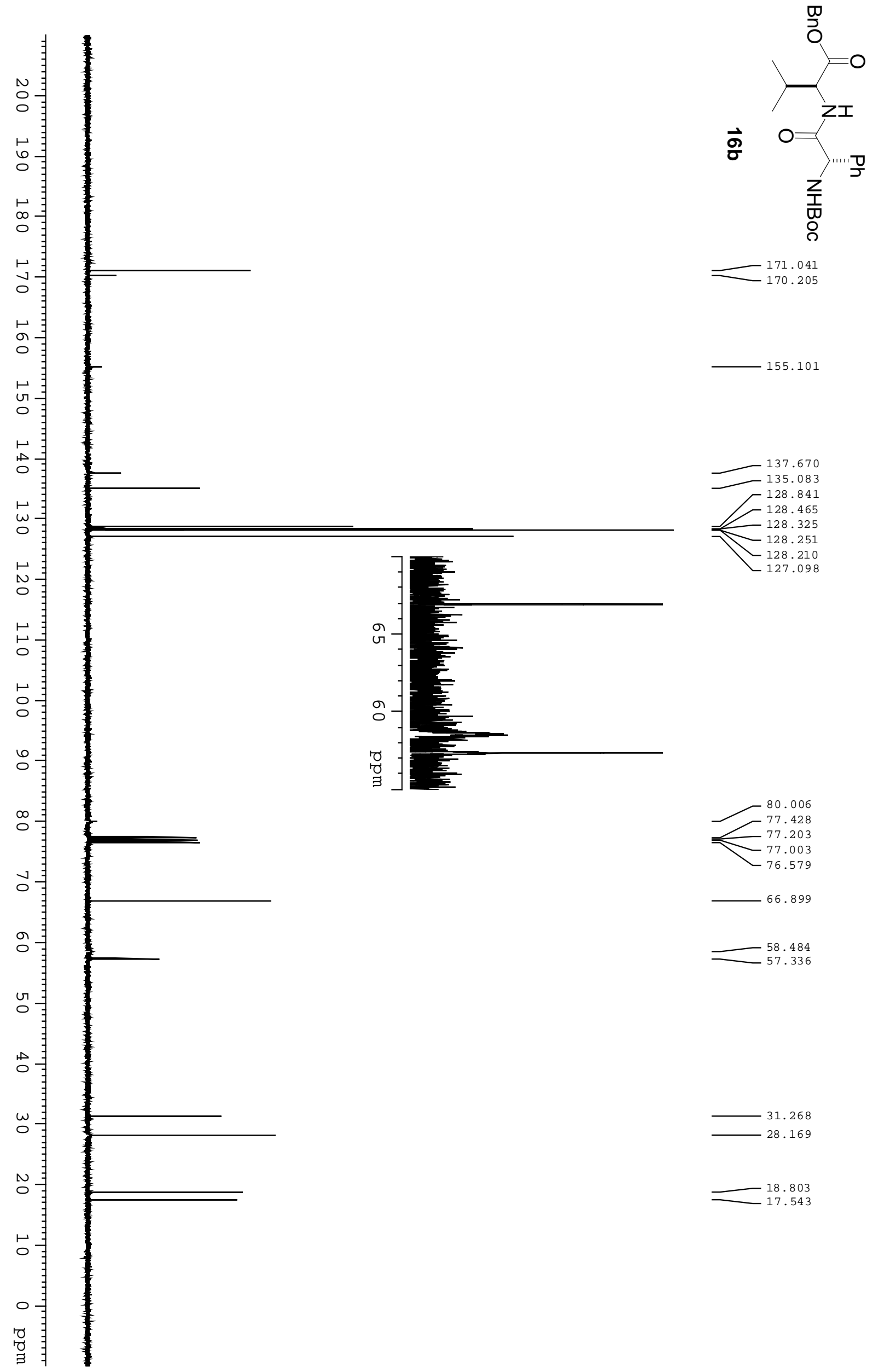

155.101
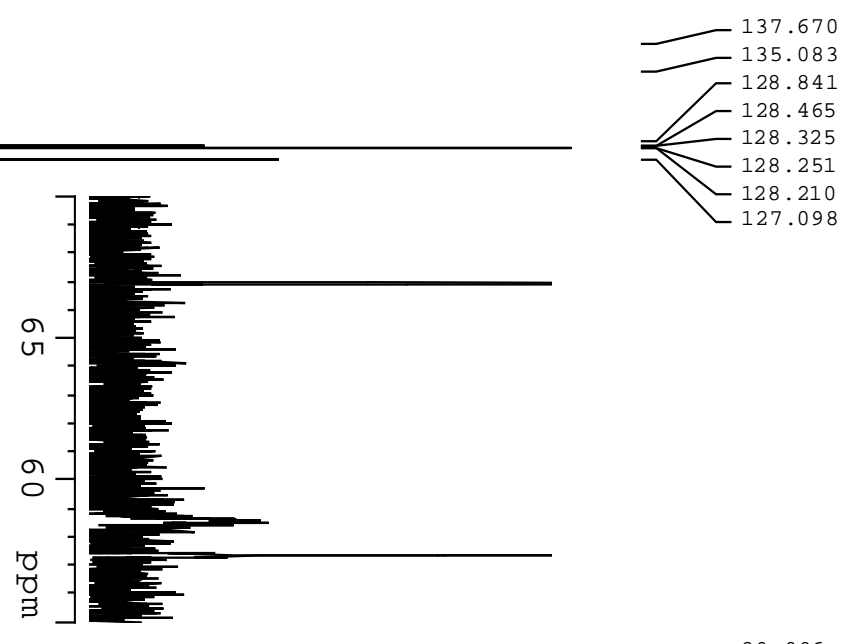

$-128.210$

$-80.006$

77.428

77.203

77.003

76.579

66.899

58.484

57.336

31.268

28.169

18.803

17.543 


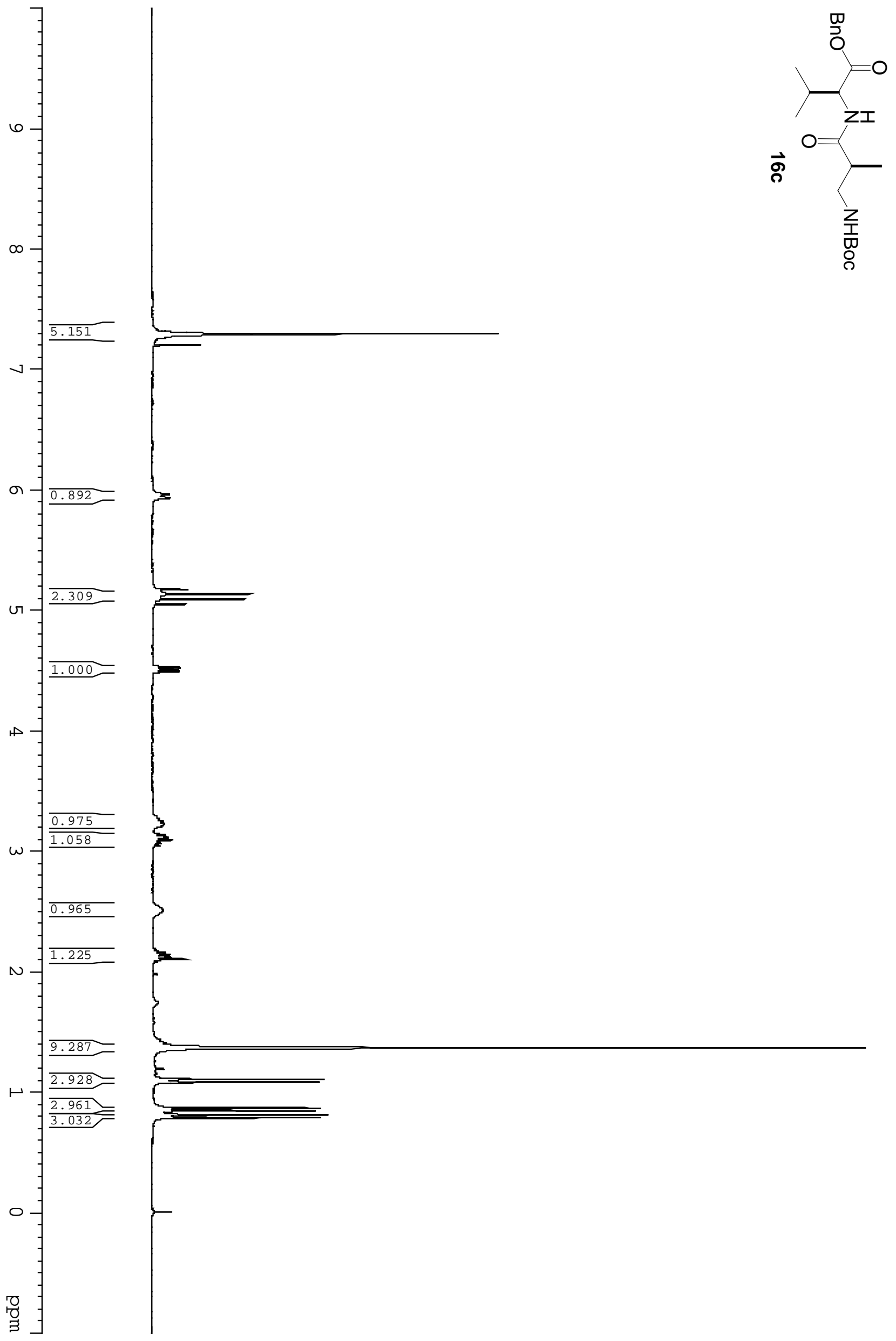




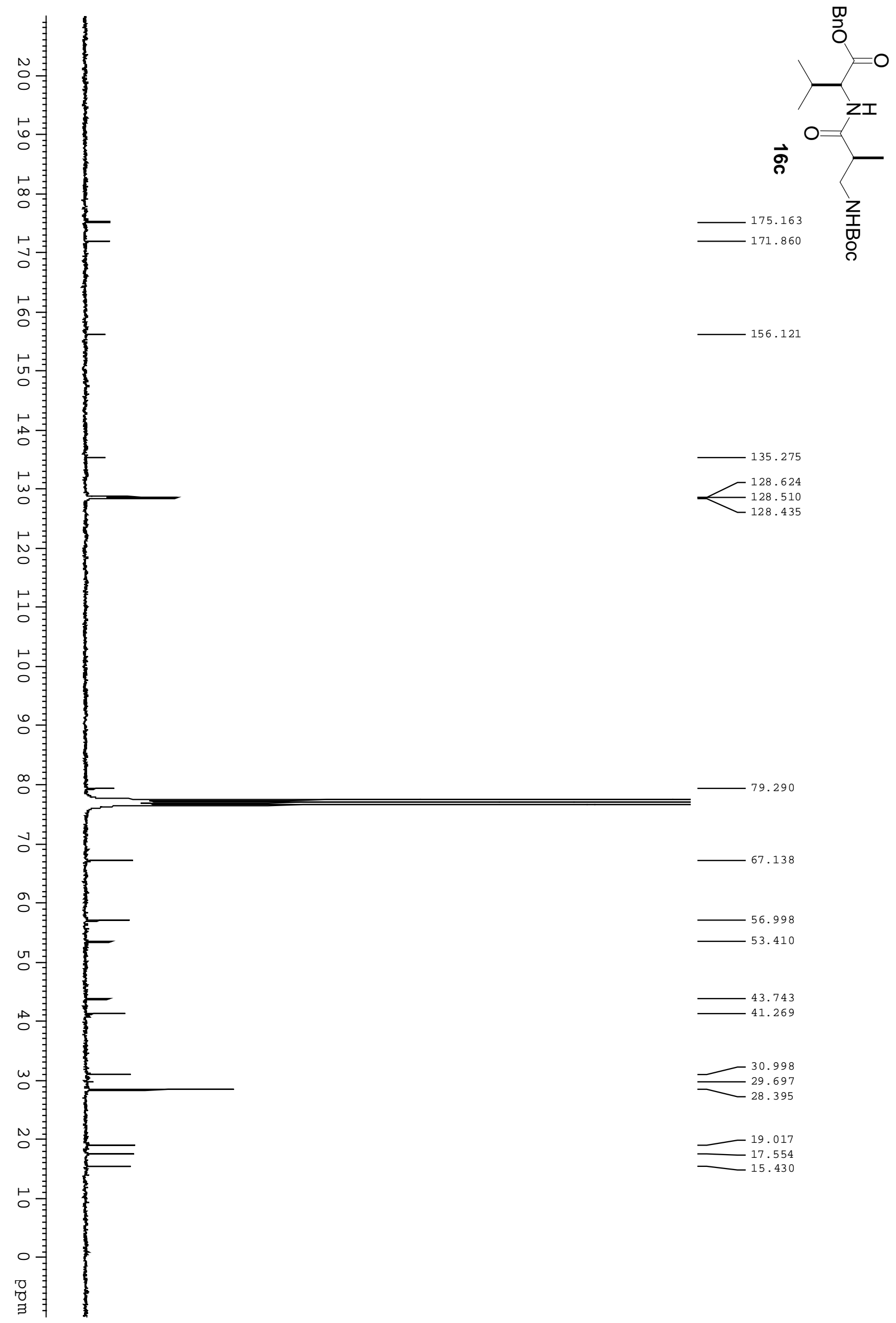



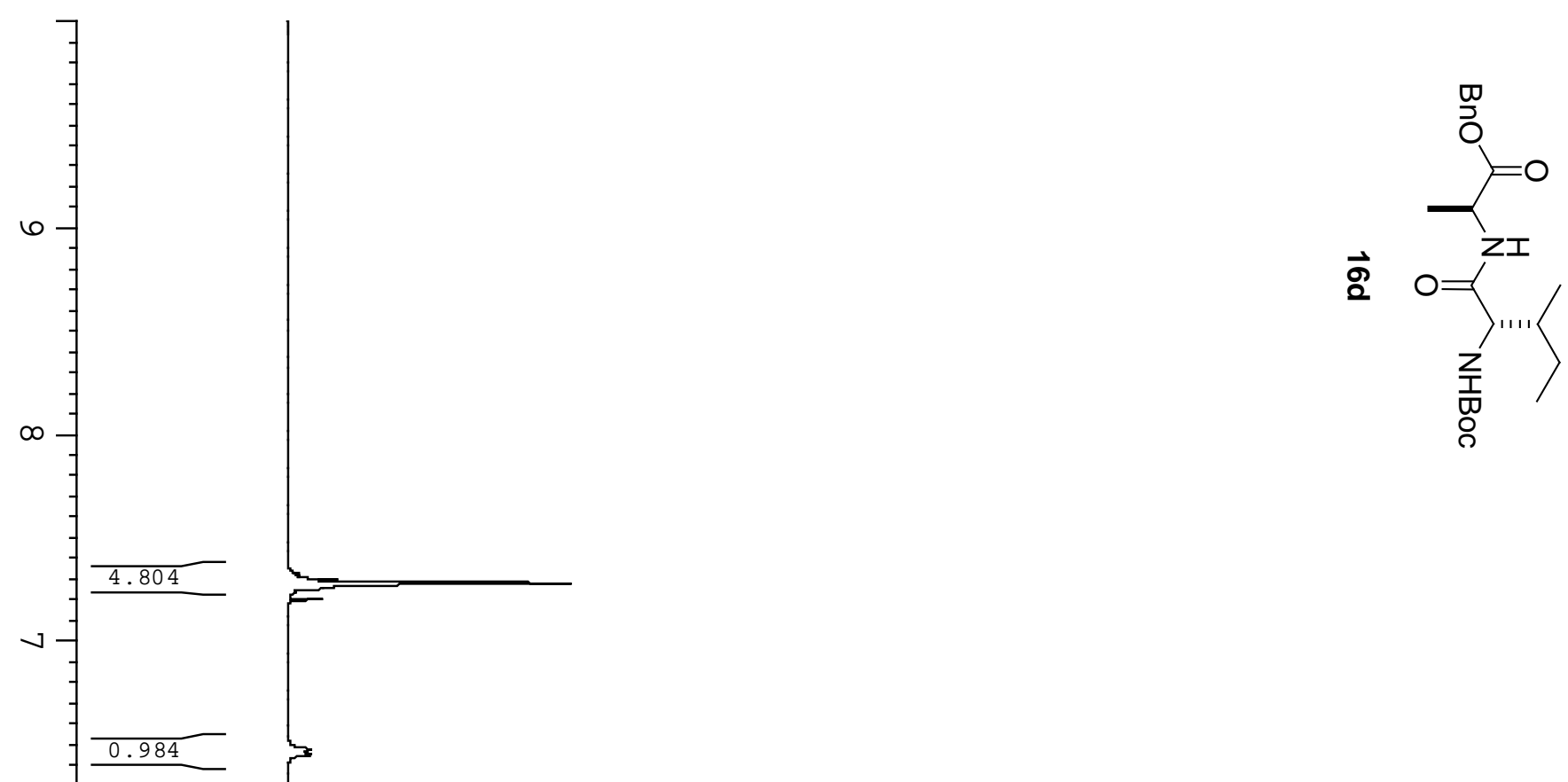

a

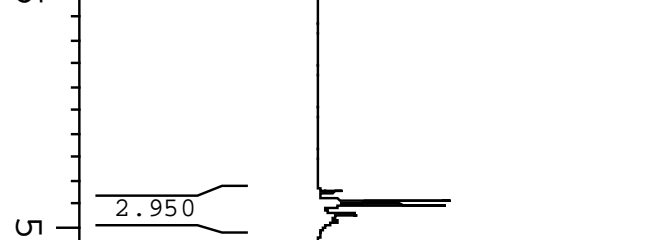
1.000

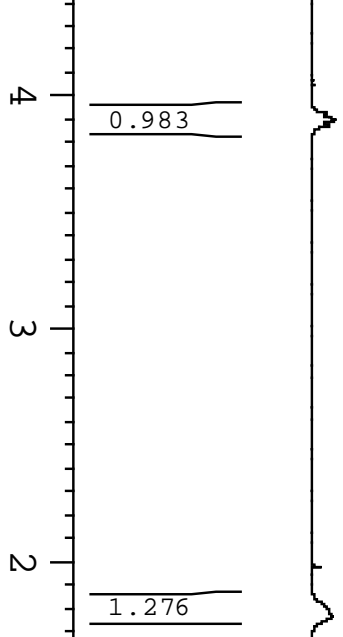

13.436

$-\sqrt{\frac{1.544}{6.286}}$

몰 

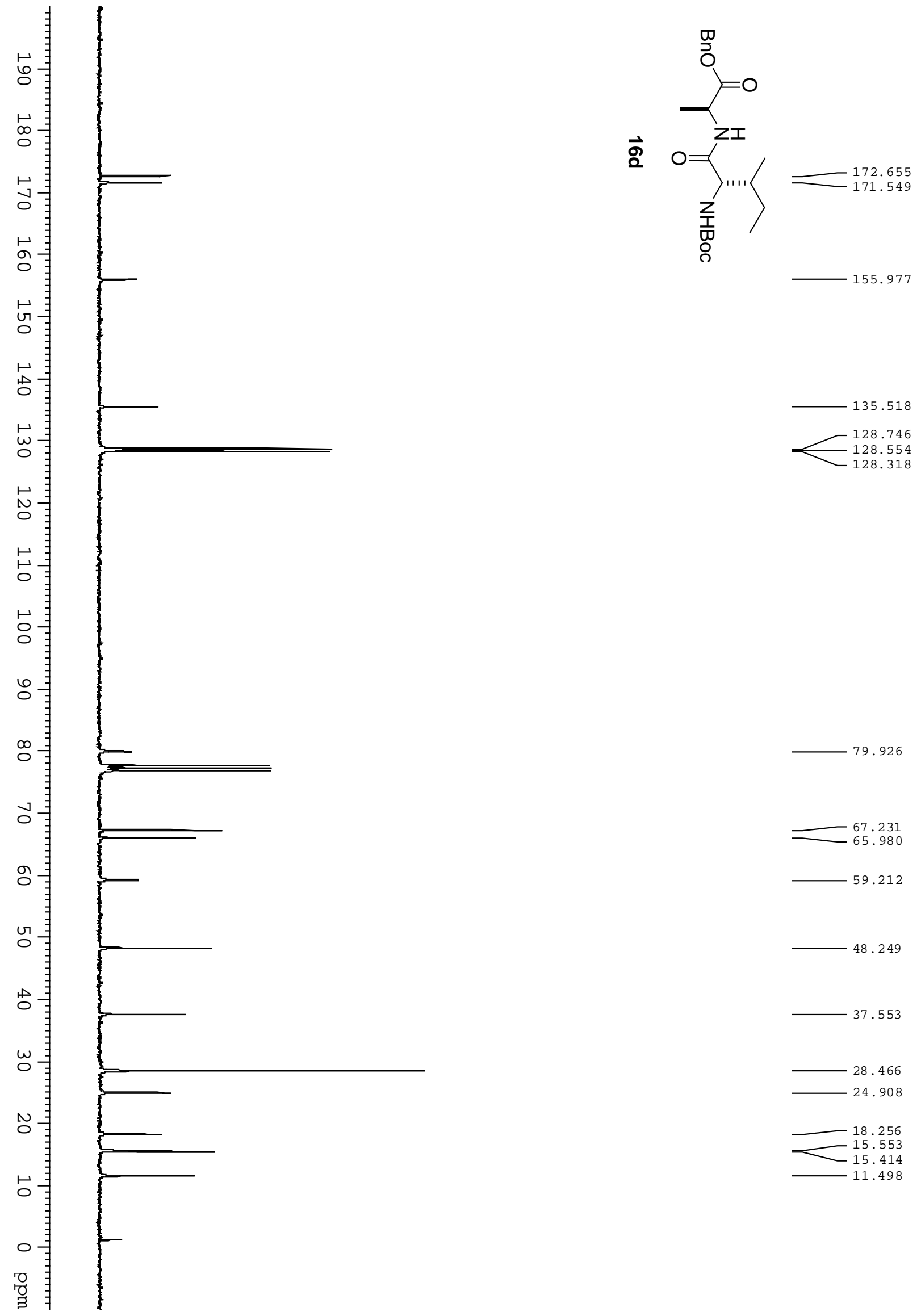


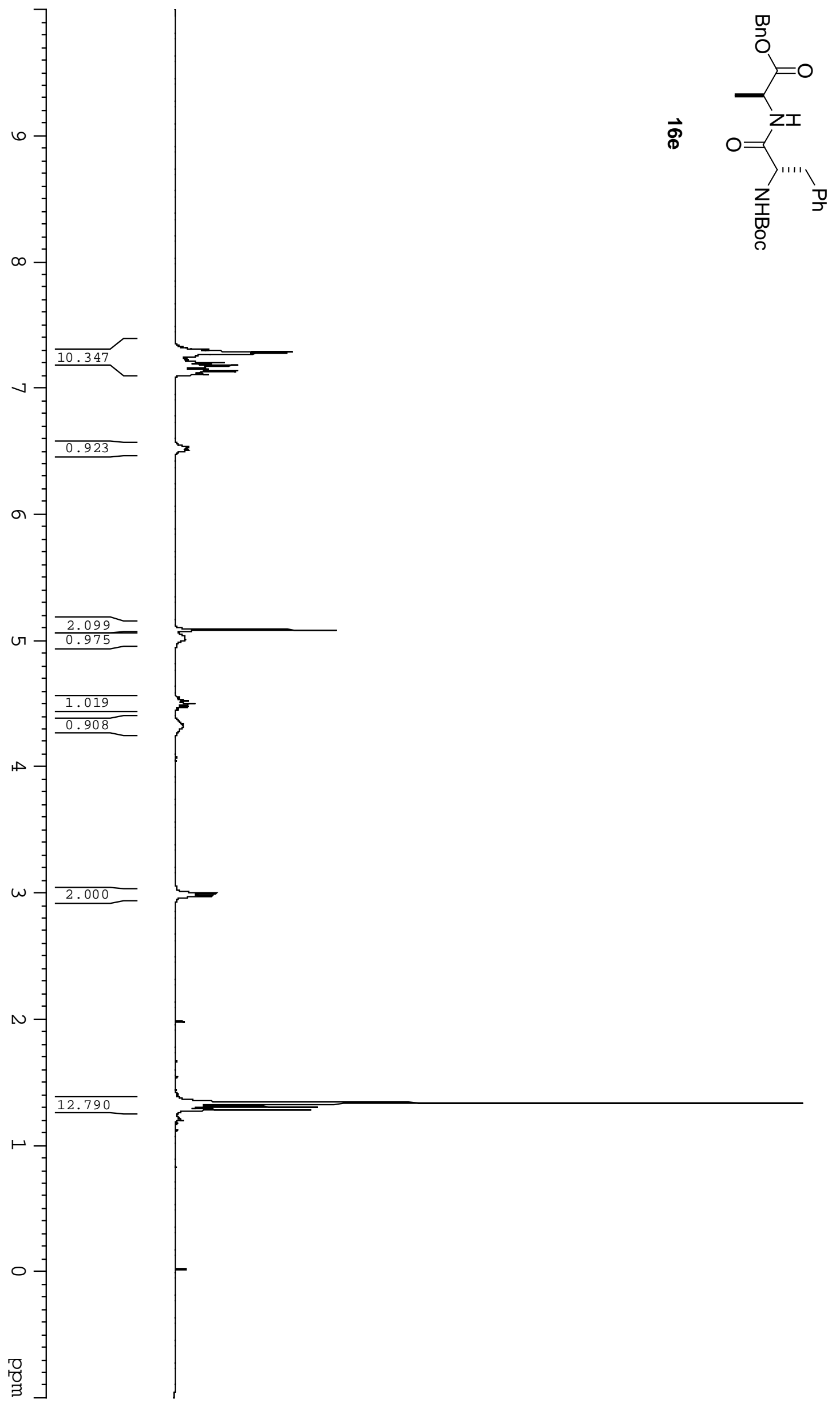



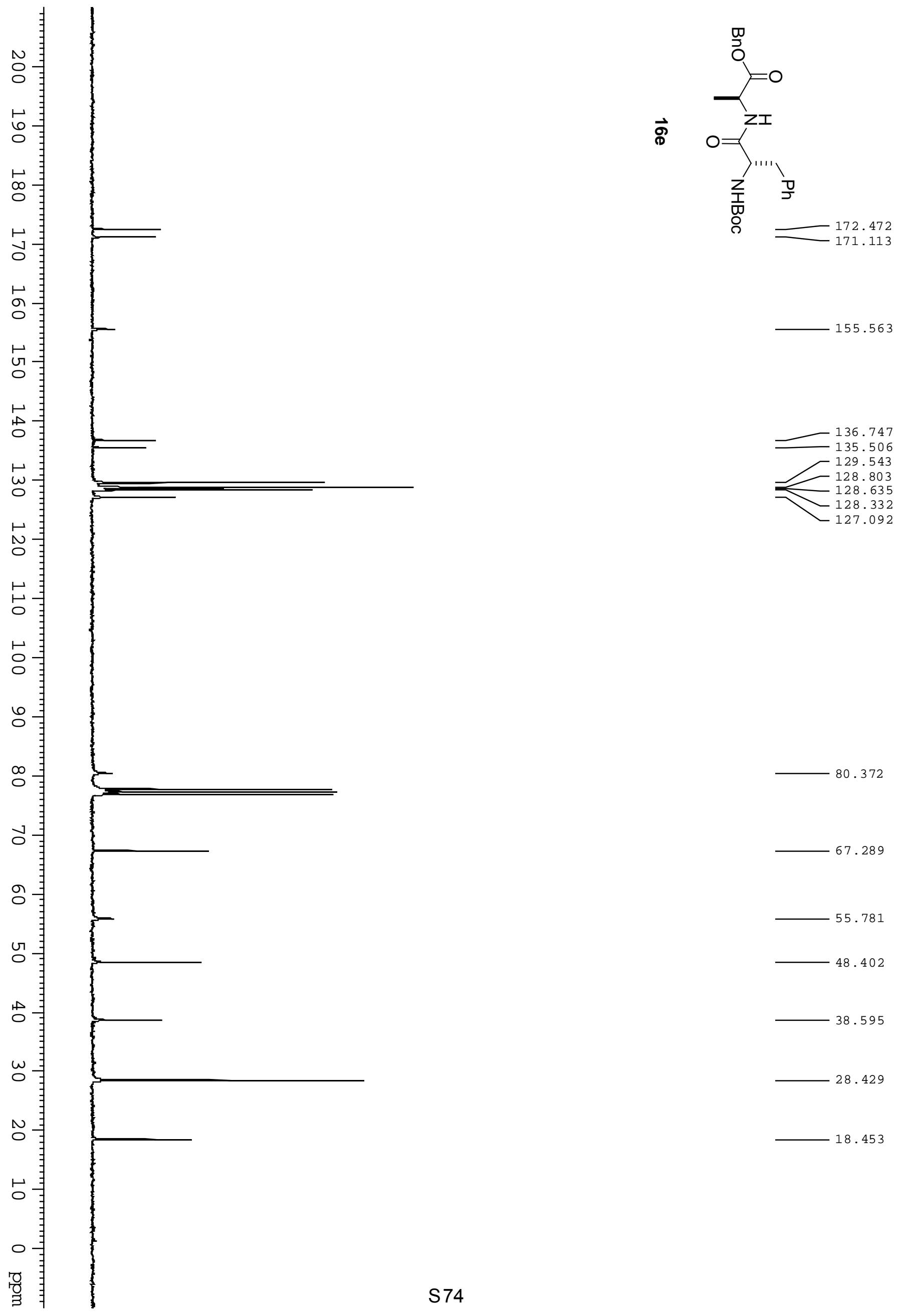

155.563
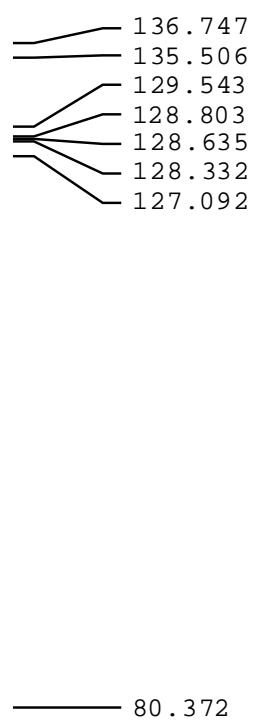

80.372

67.289

55.781

48.402

38.595

28.429

18.453 

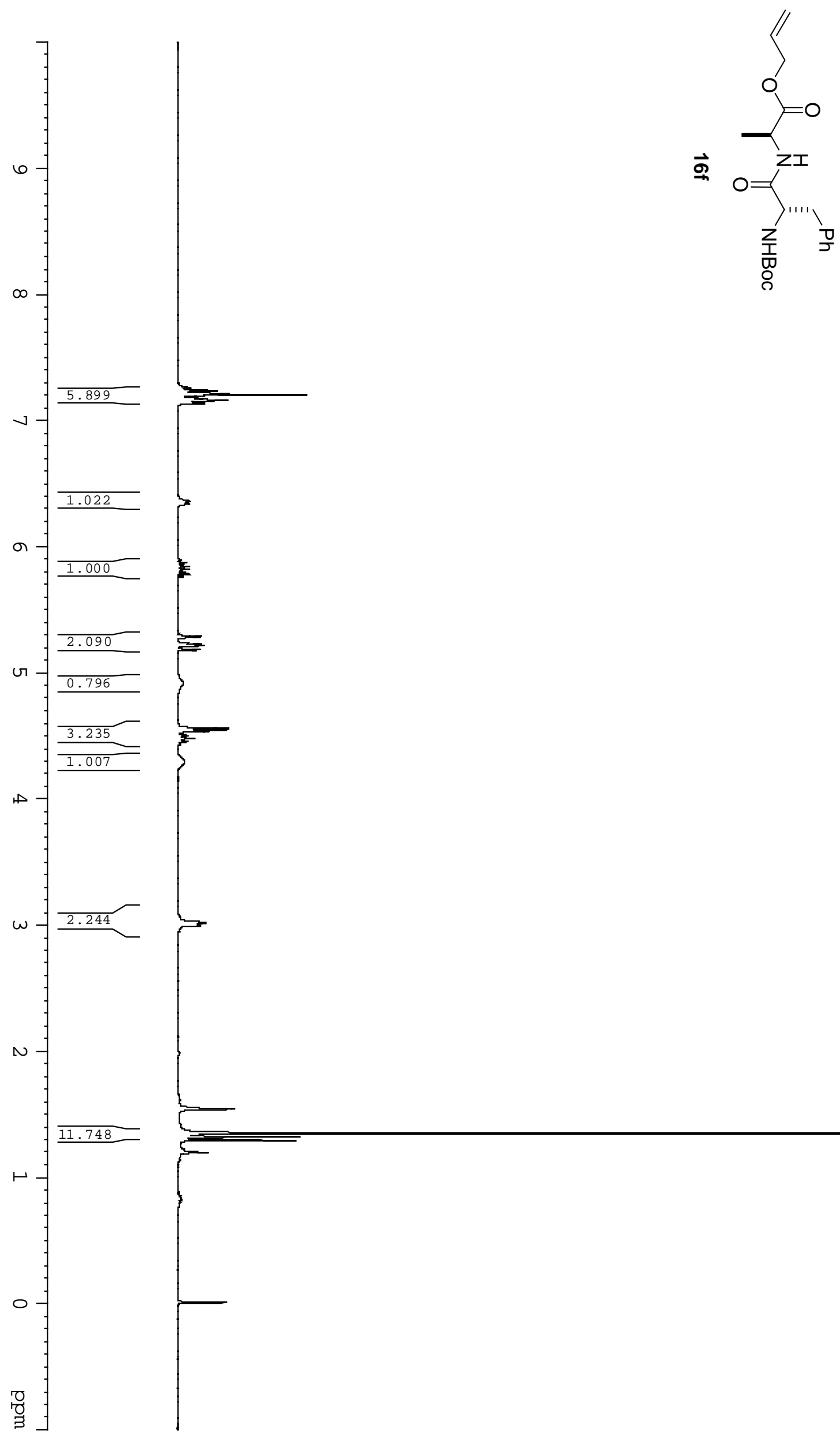


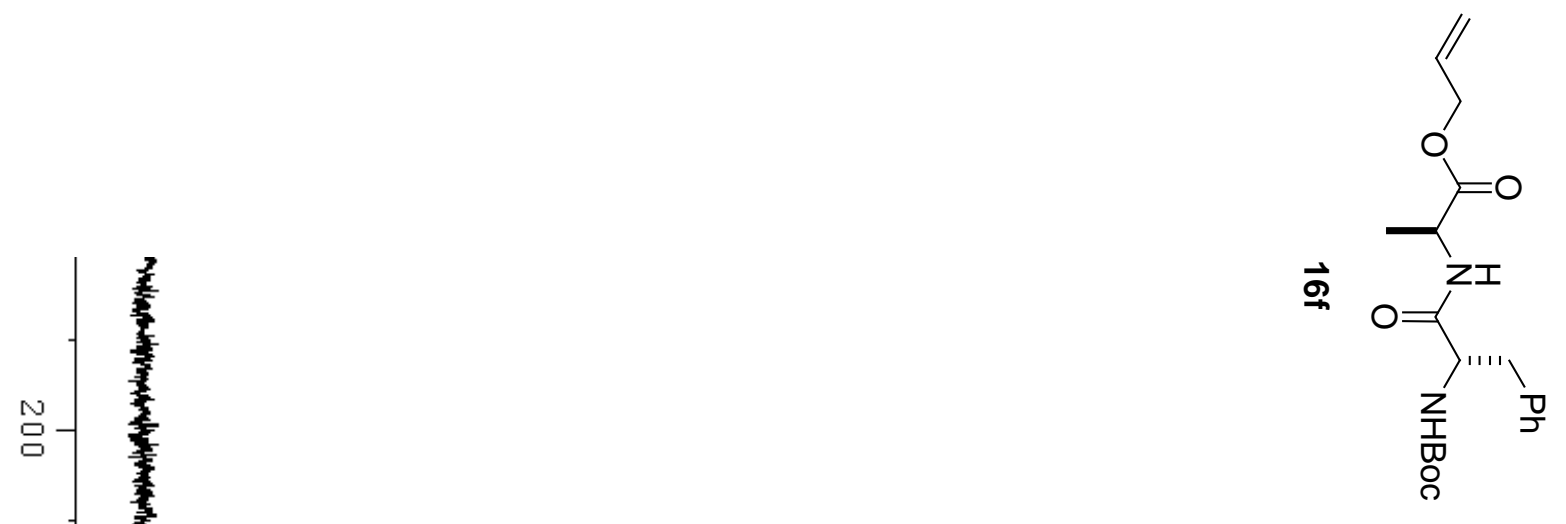

$\stackrel{\circ}{\circ}$

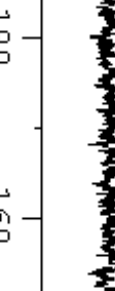

里

$\stackrel{\bullet}{\bullet}$

令

$\stackrel{\stackrel{N}{口}}{ }$

宫

器

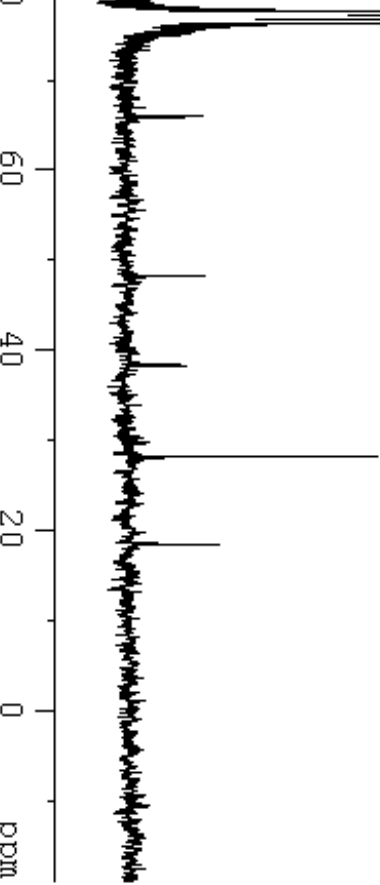

80.230

65.951

48.202

38.323

28.239

18.454 


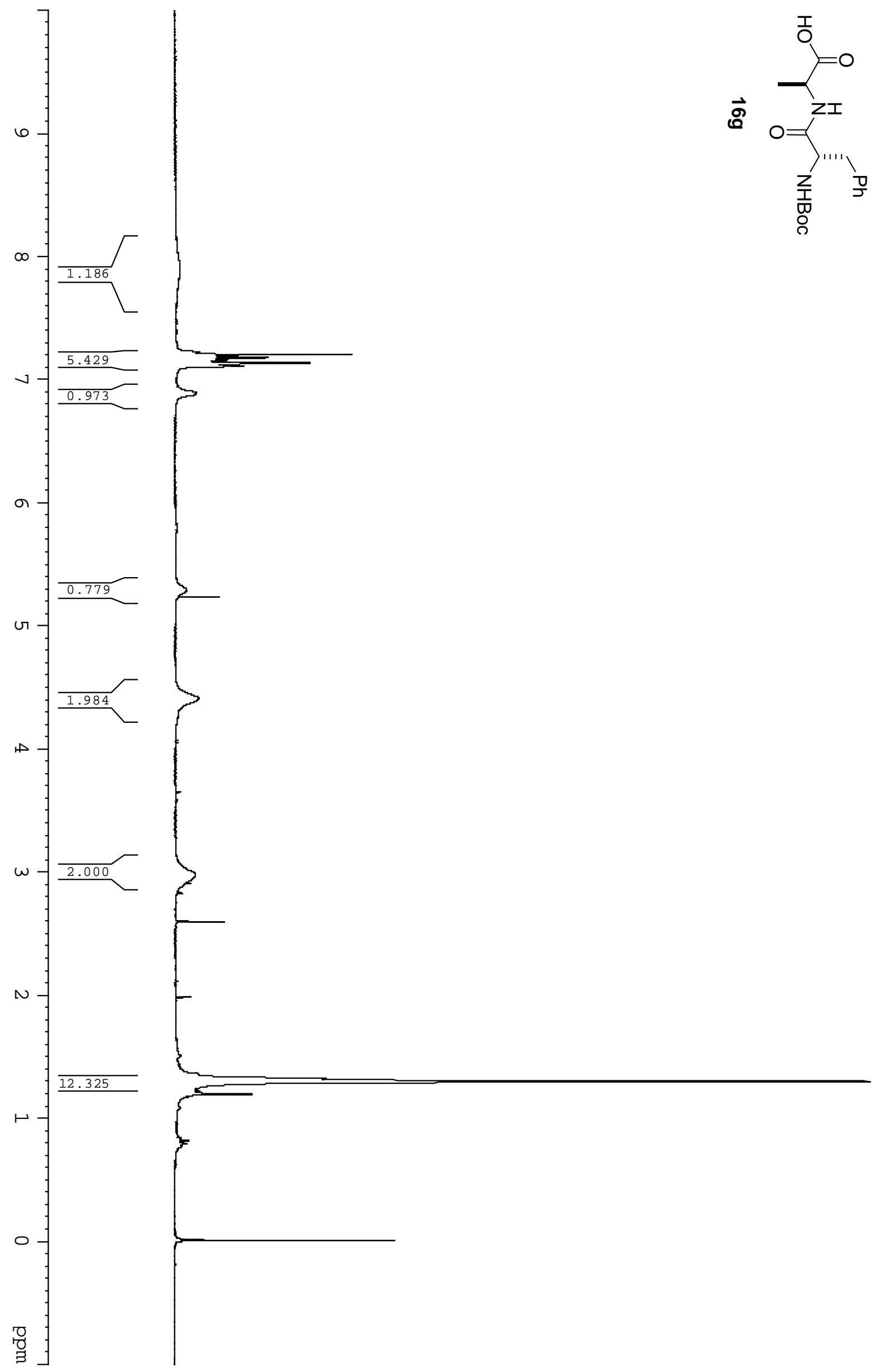




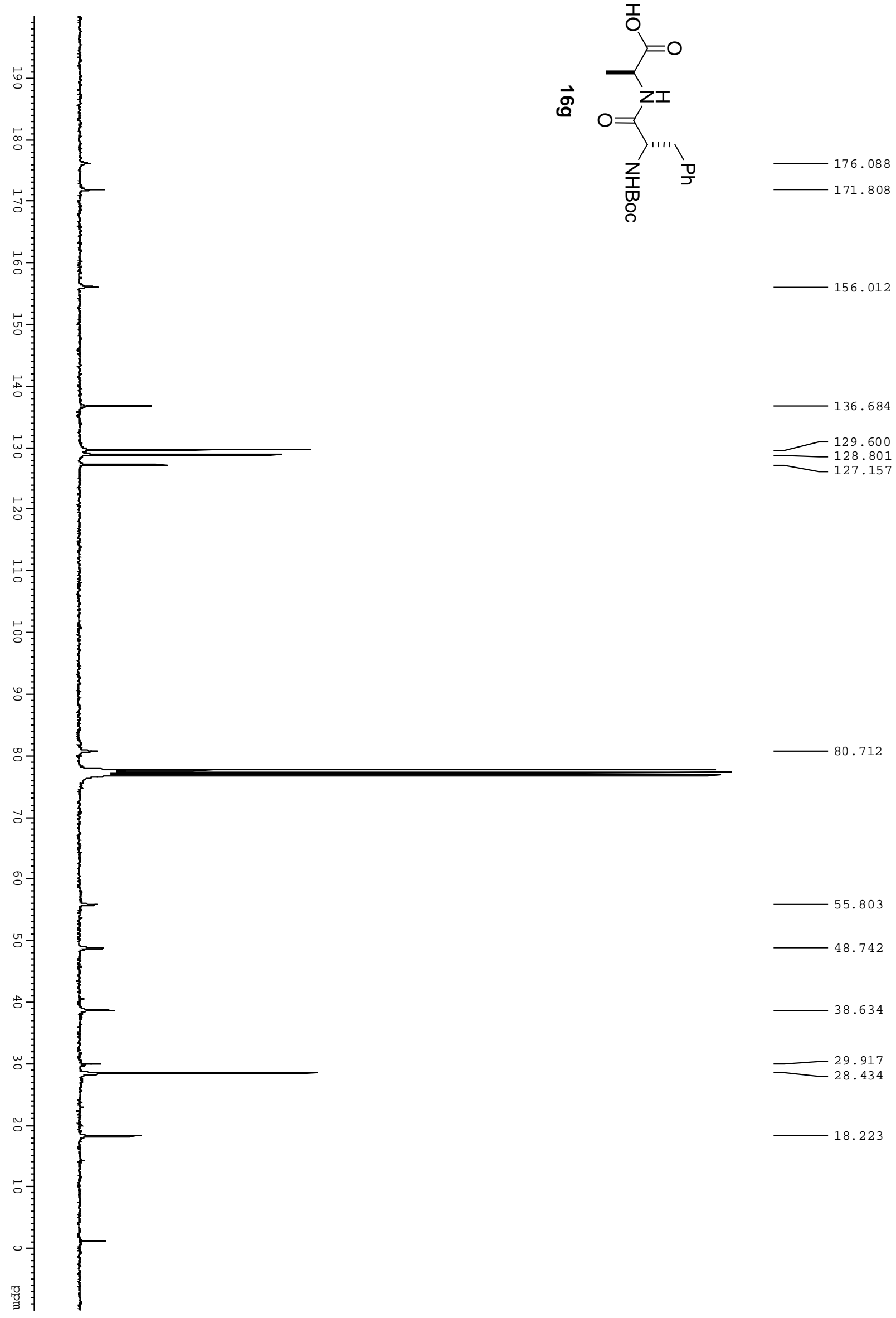




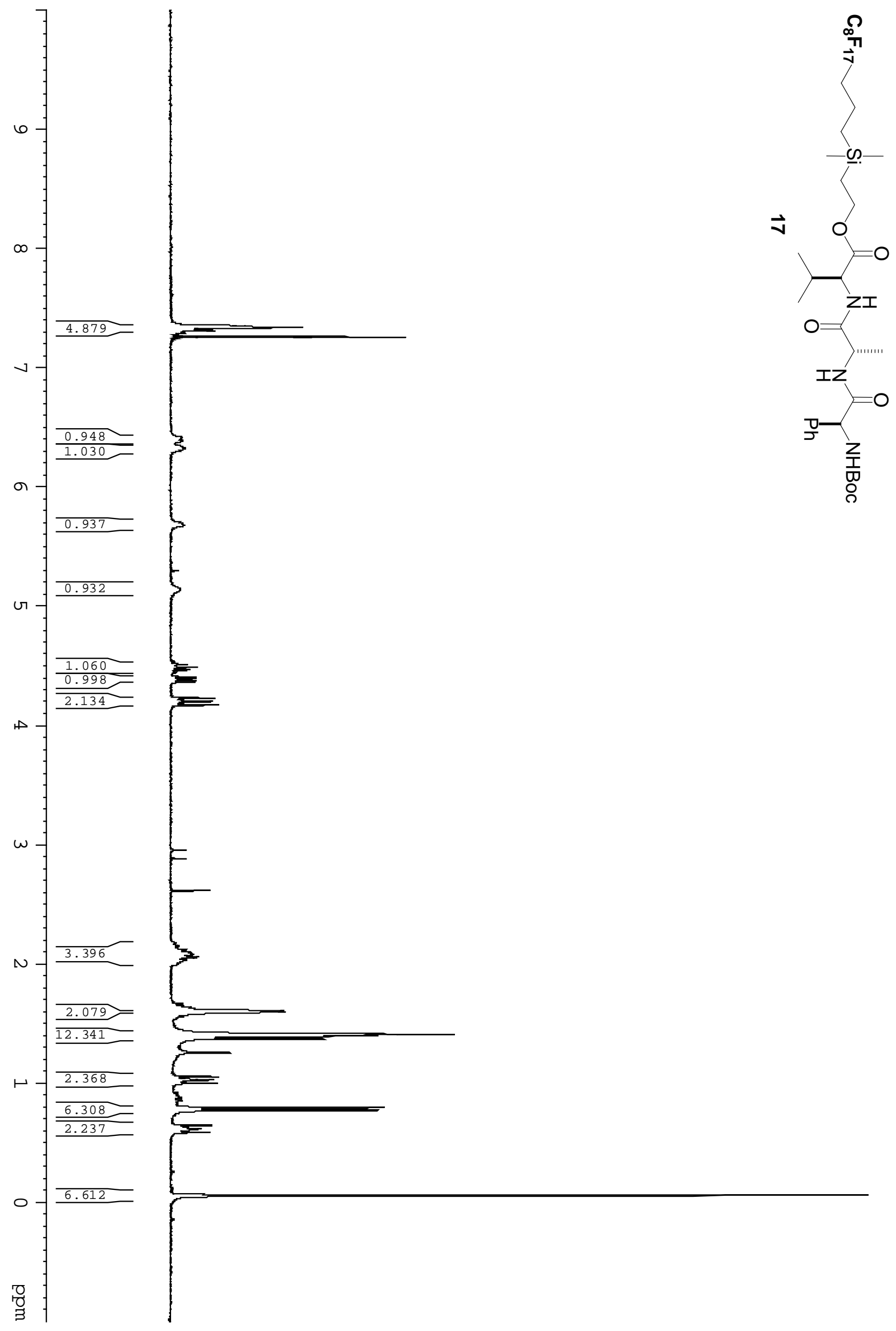




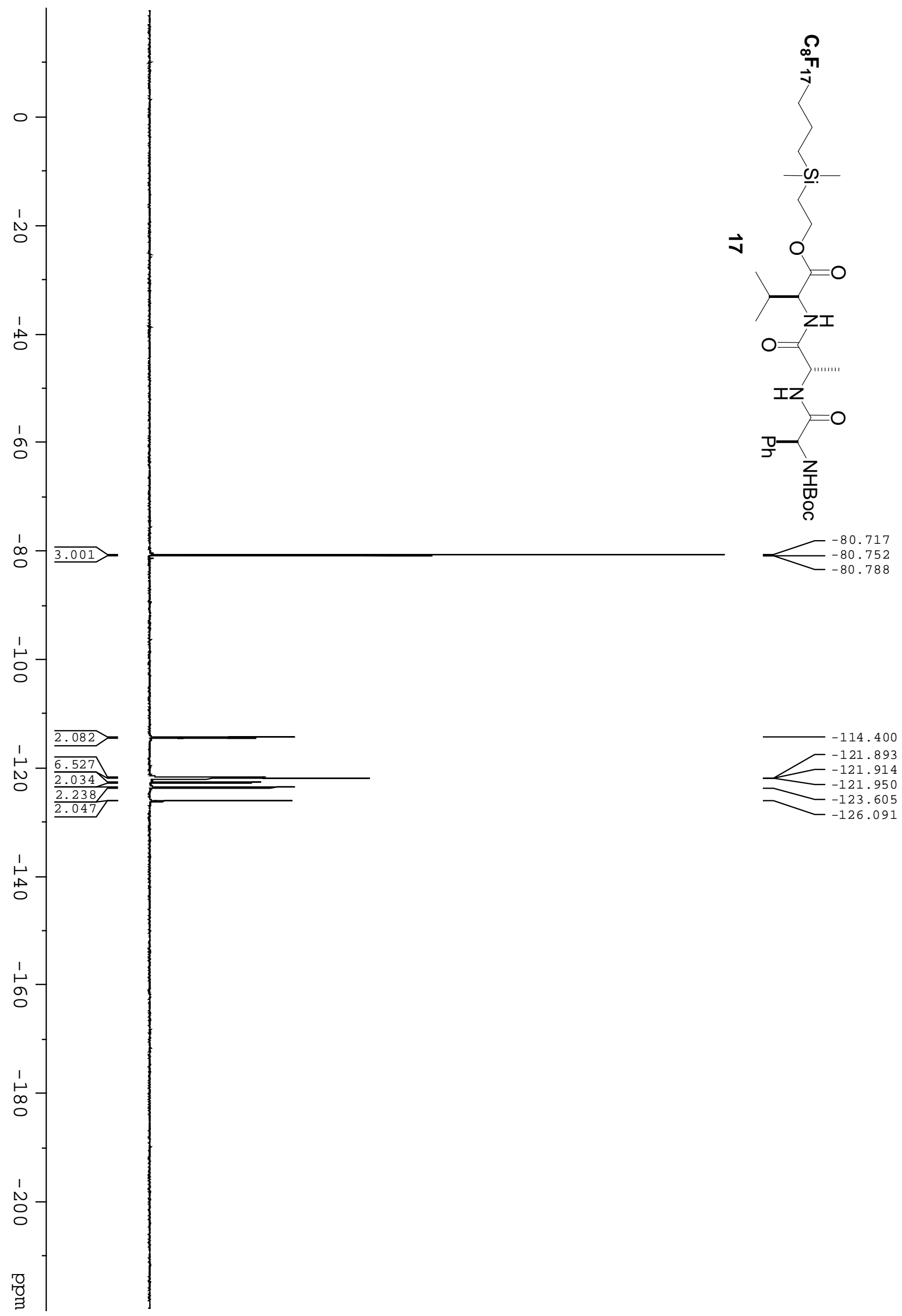



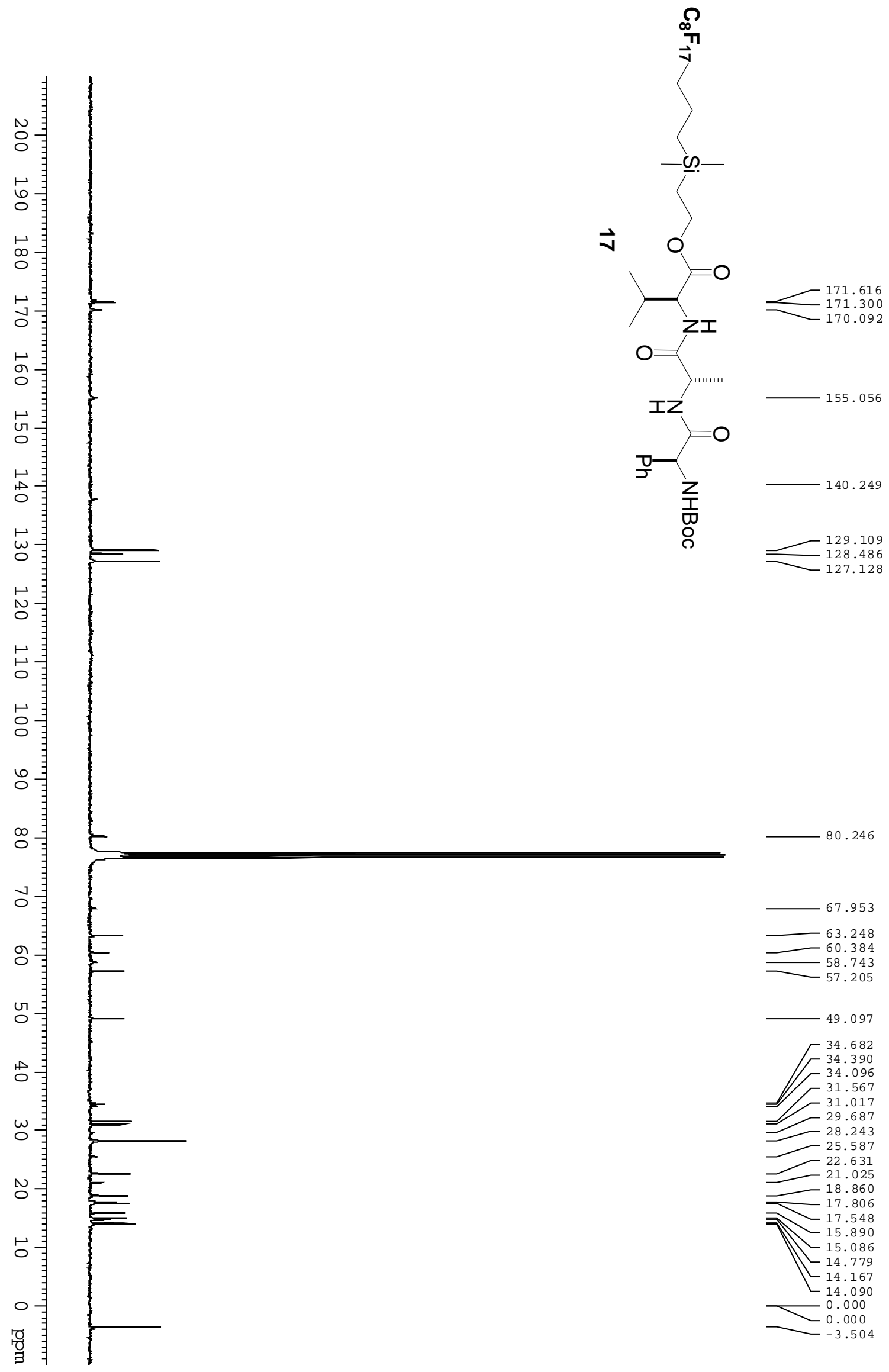


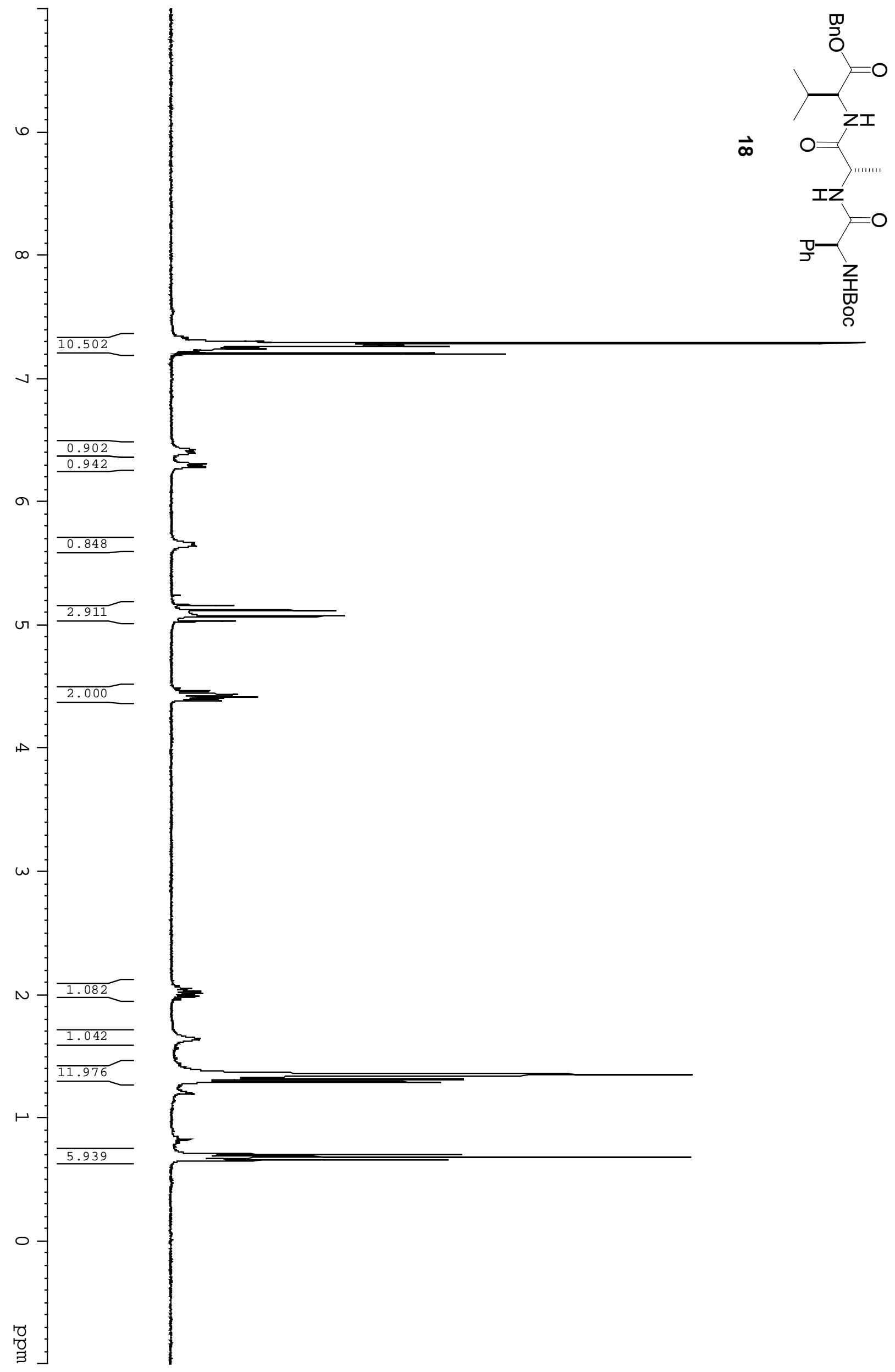



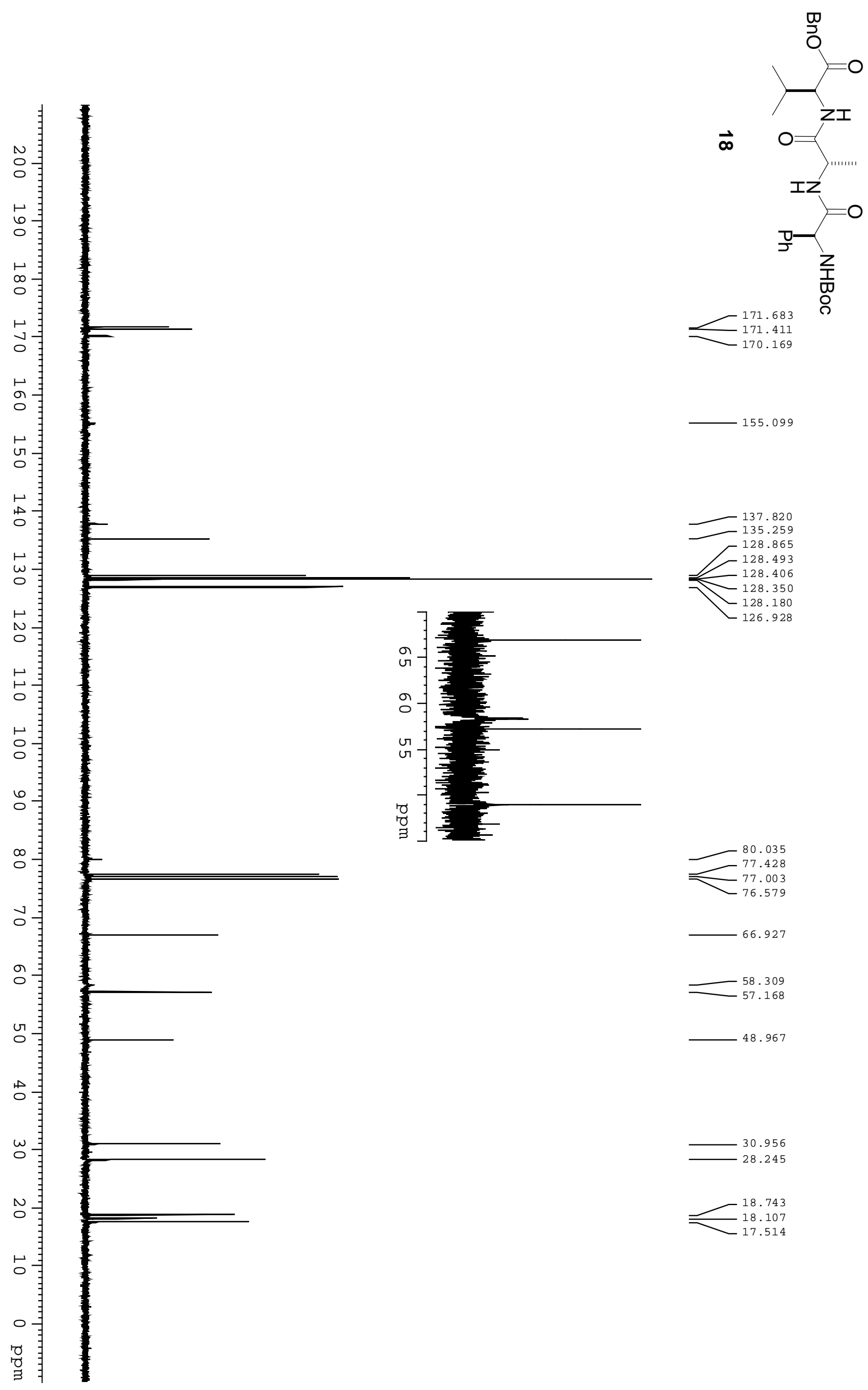


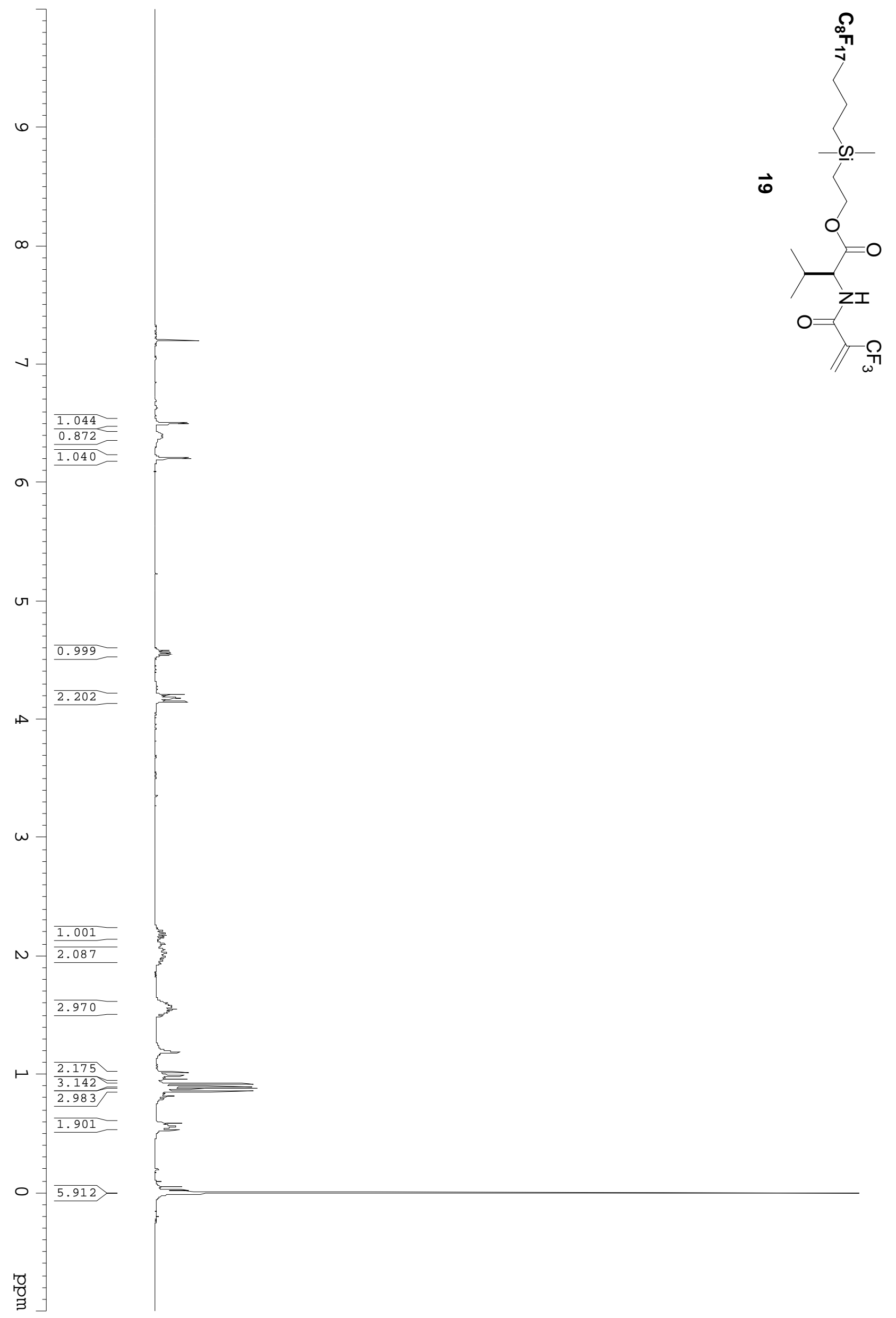



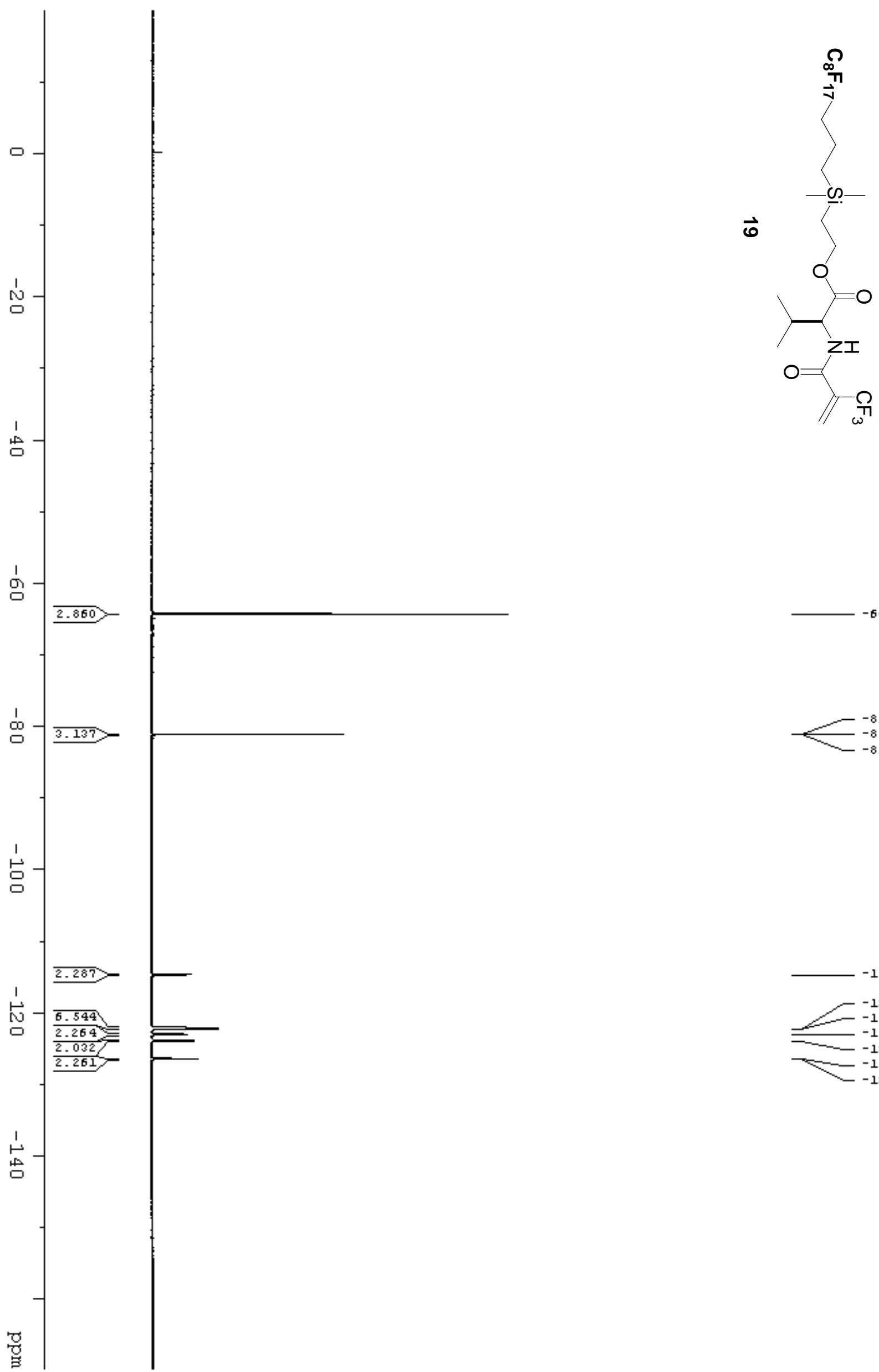

$-64.306$

$-81.119$

$-81.154$
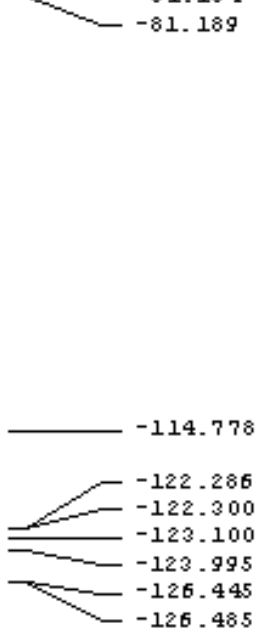


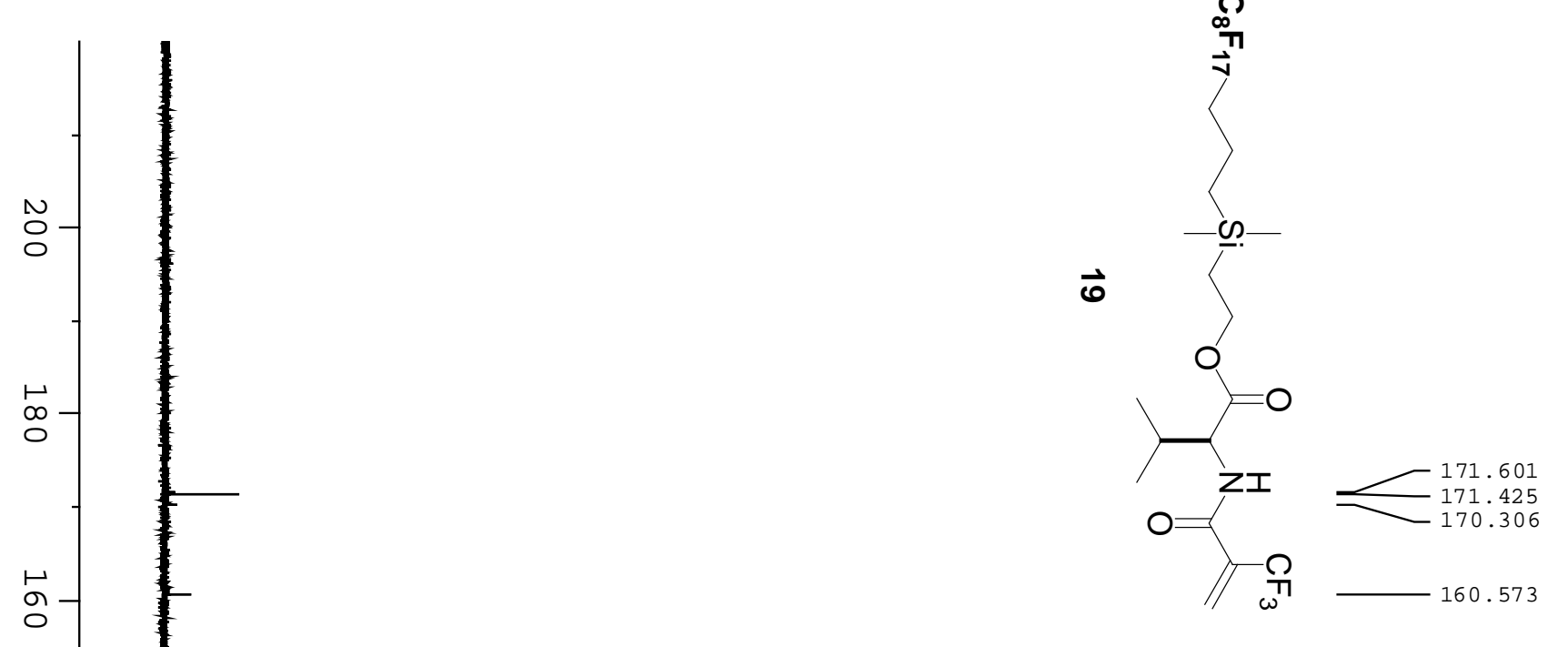

63.576

58.754

$\begin{array}{r}58.754 \\ -57.522 \\ \hline\end{array}$

8

$\stackrel{\circ}{\circ}$

$\stackrel{\infty}{\circ}$
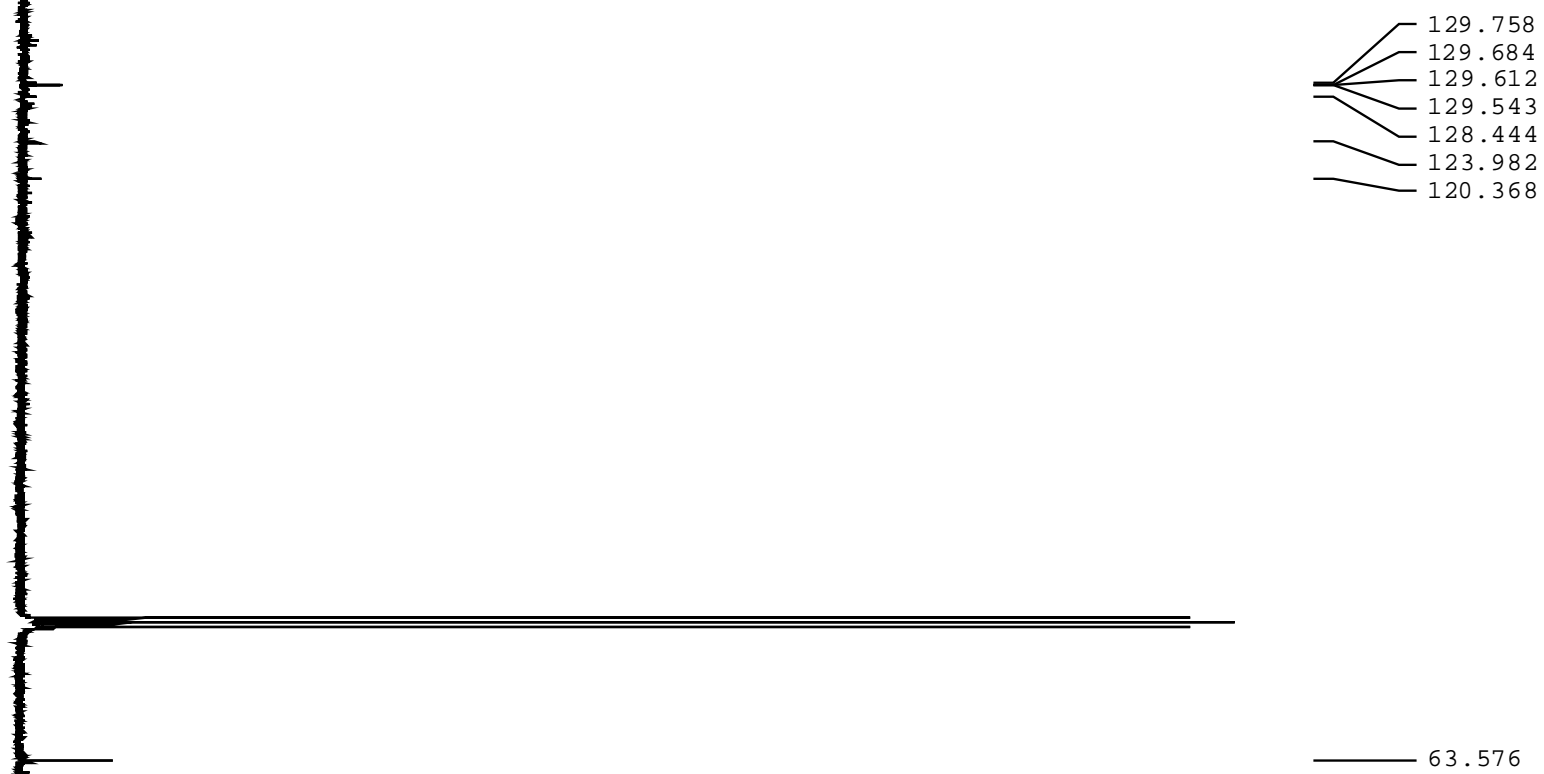

(1)

古
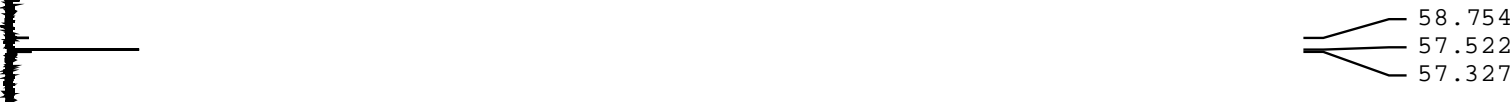

$-34.704$ F 34.411

34.118

- 31.921

$-31.386$

$-31.290$

31.092

31.092
29.692

$-29.355$

22.683

19.040

18.906

- 17.921

$\longrightarrow 17.578$

$-15.939$

$-15.097$

-14.800
-14.743

$-14.743$

$-14.097$

$\odot-$

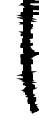

몰 


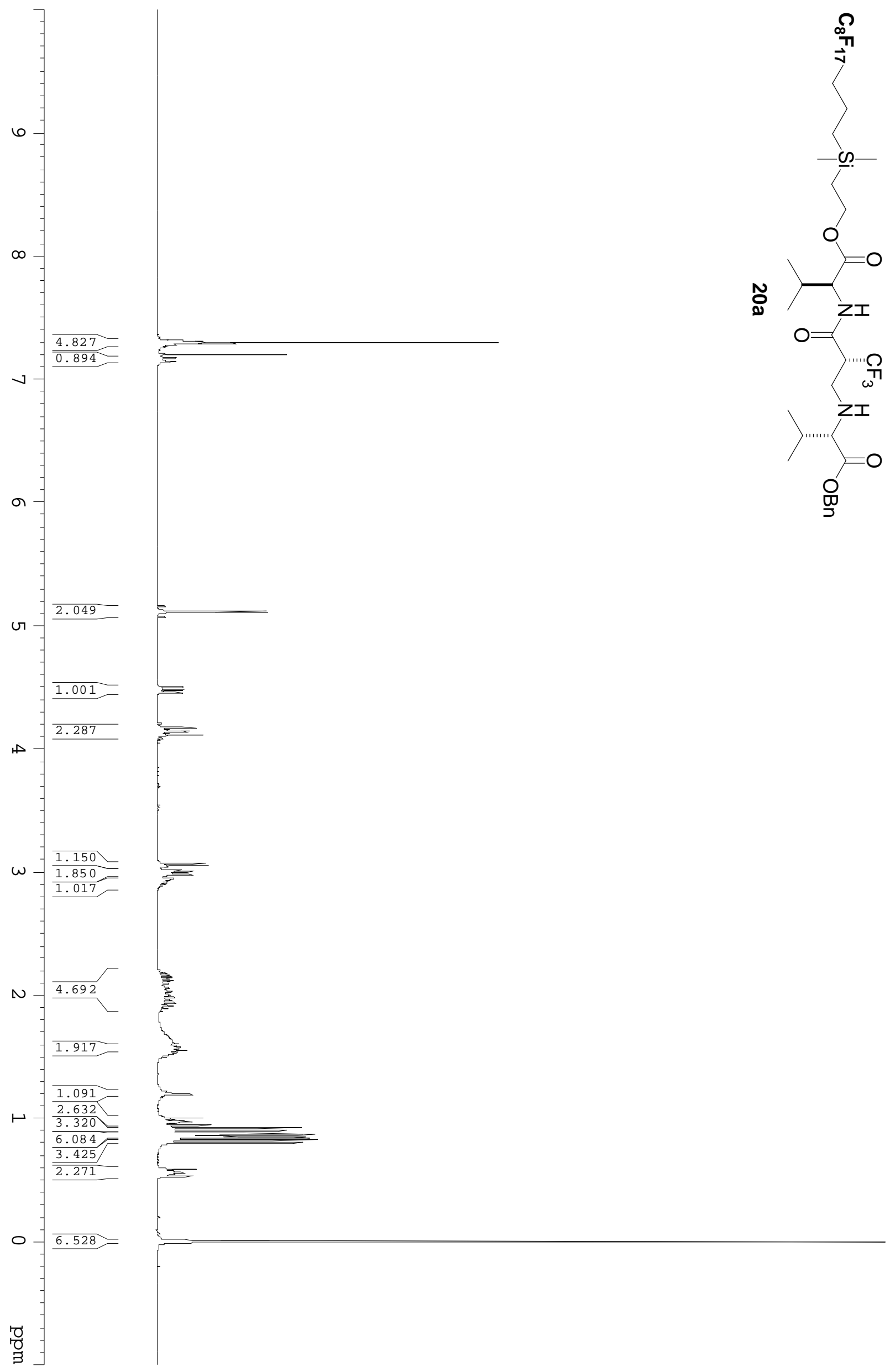




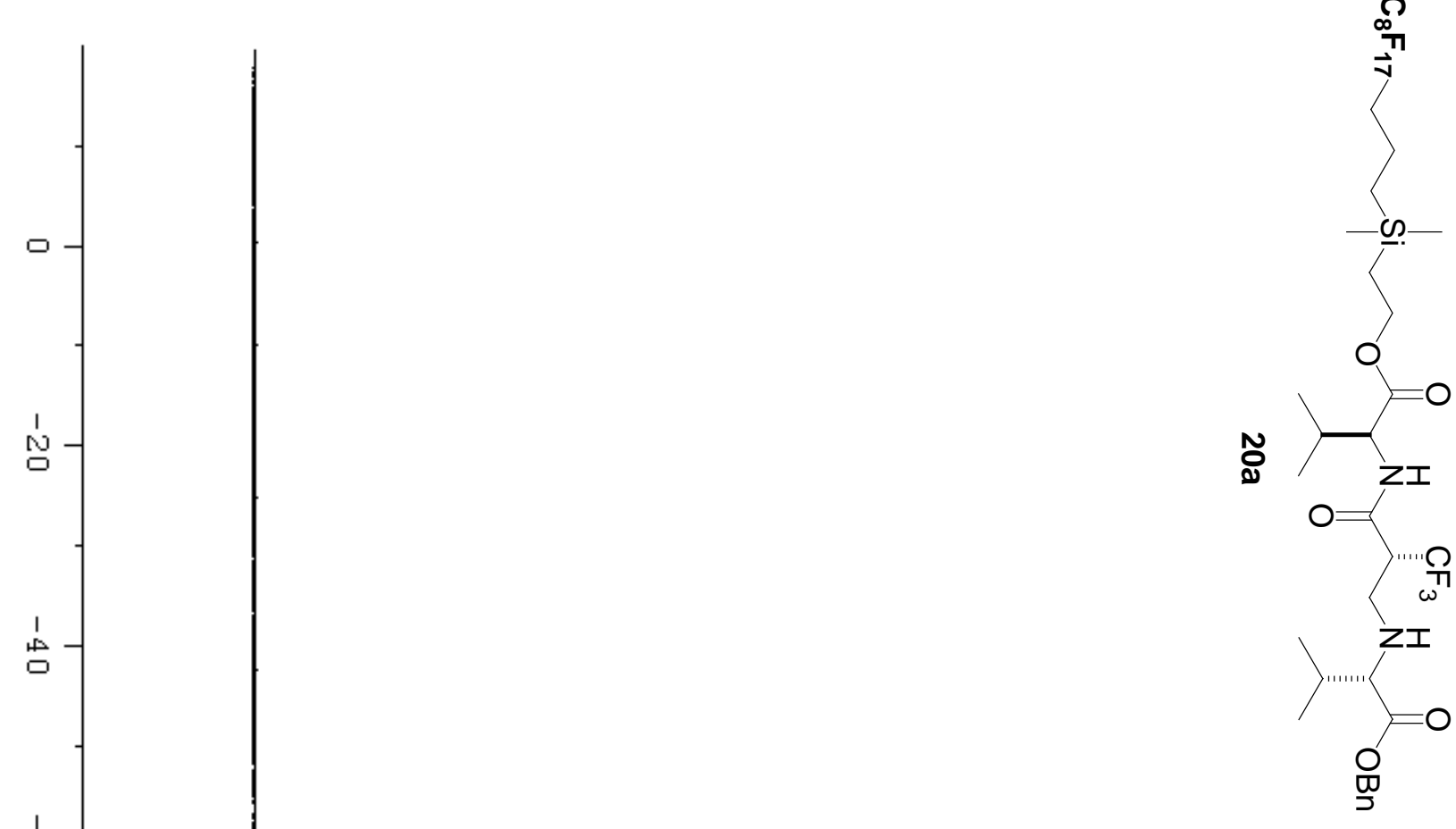

$\stackrel{1}{\circ}$

읨

吕
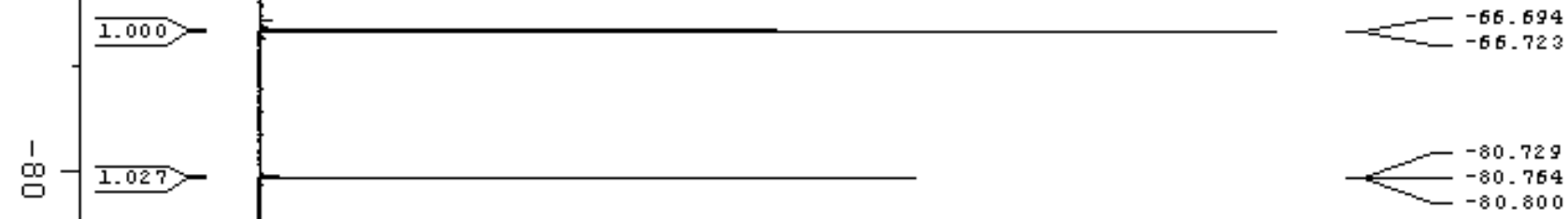

$-66.723$

$\stackrel{\text { I }}{口}$

$0.747=$

$\stackrel{!}{口}-\frac{\frac{0.214}{0.731}}{\frac{0.734}{0.709}}$

$0.709 /$

$\stackrel{\stackrel{1}{\bullet}}{\stackrel{4}{口}}$
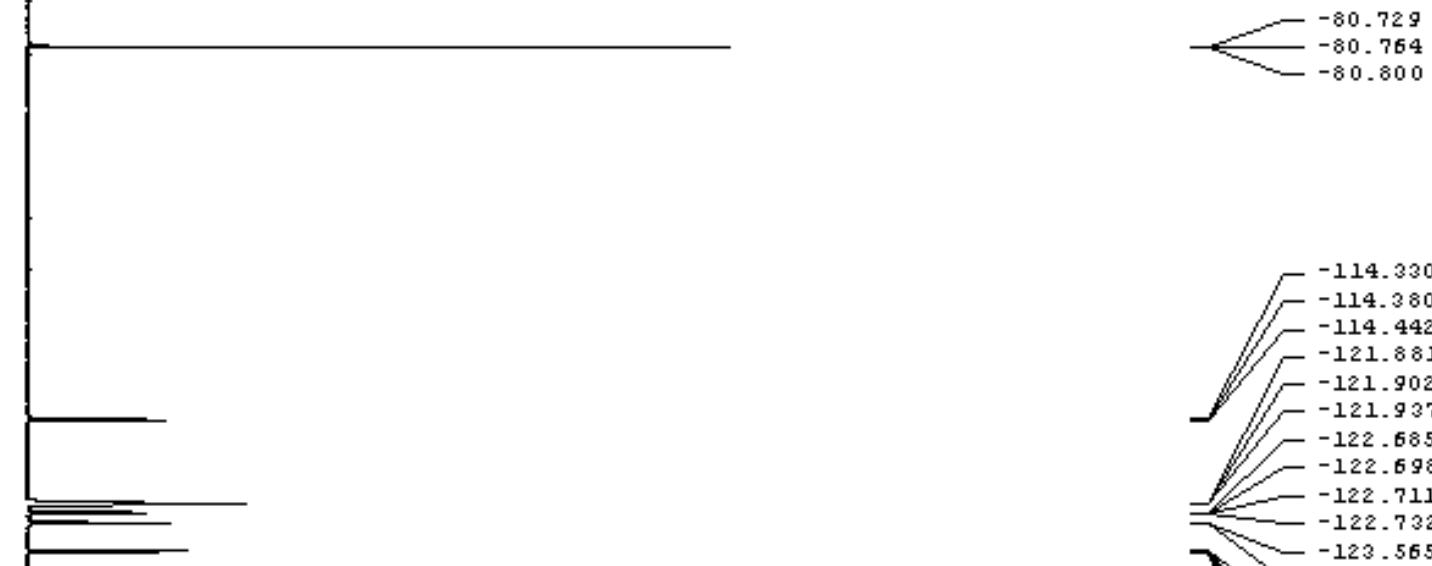

몸

$\stackrel{\stackrel{\bullet}{\bullet}}{\stackrel{9}{口}}$

g
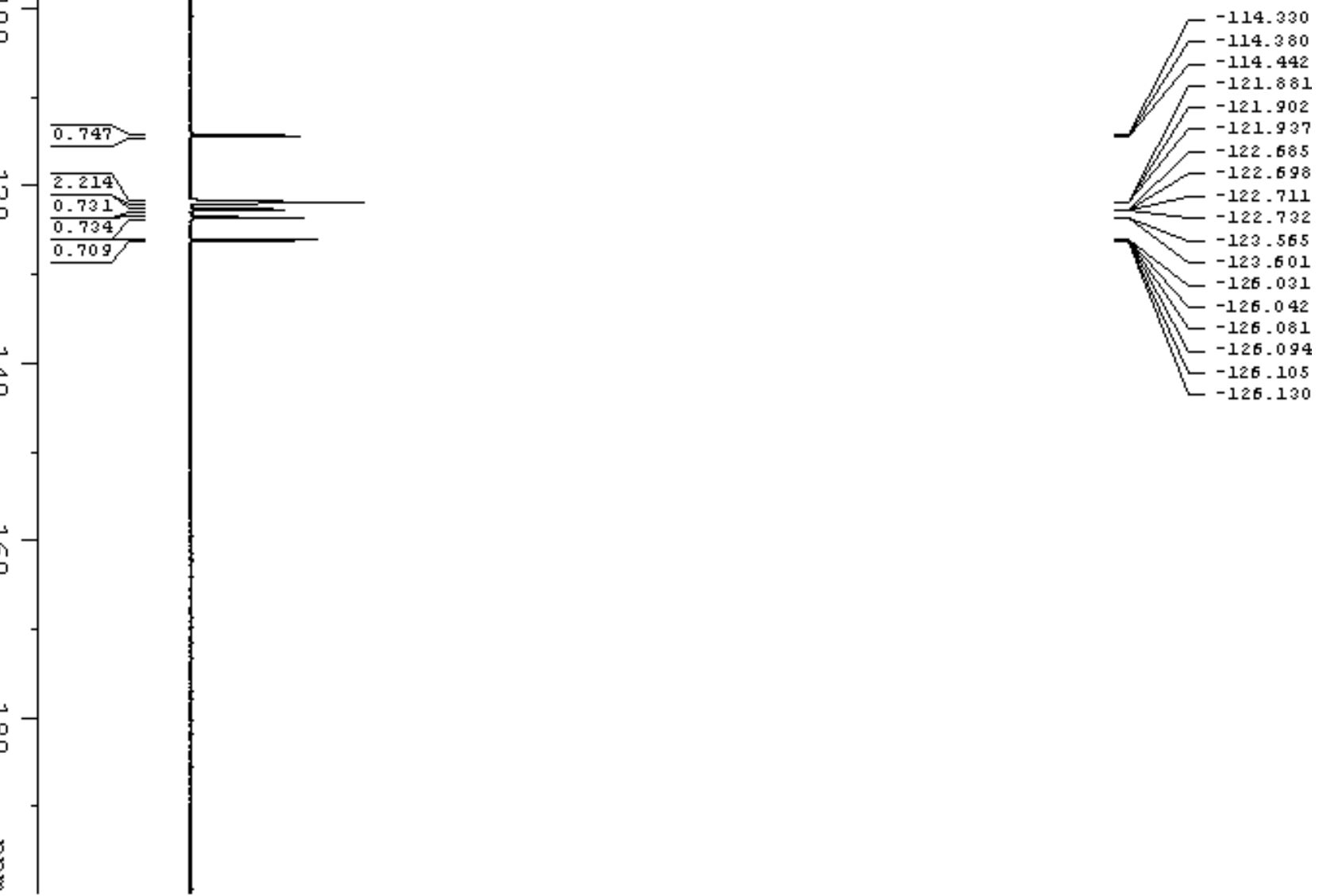


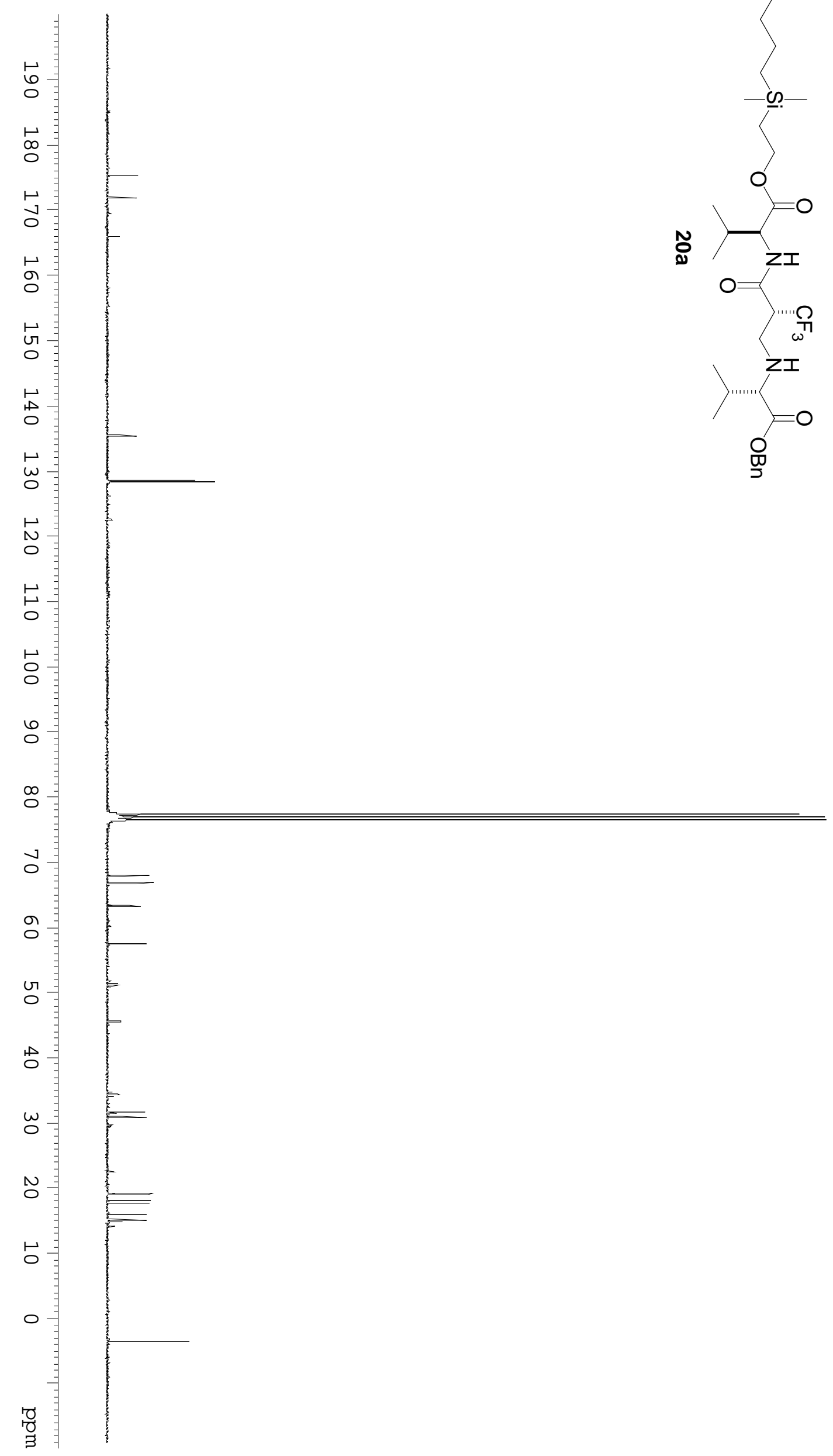

135.514 129.982 128.625

128.481 128.439 126.269 122.555

67.913 66.785 63.284 57.483 51.772 51.441

51.109 50.776 45.538 45.504 34.706 34.416 34.125 31.667
34.629 30.929 19.124 19.055 18.115 17.641 15.925 15.110 14.854 14.803 $-14.748$ $-3.493$ 


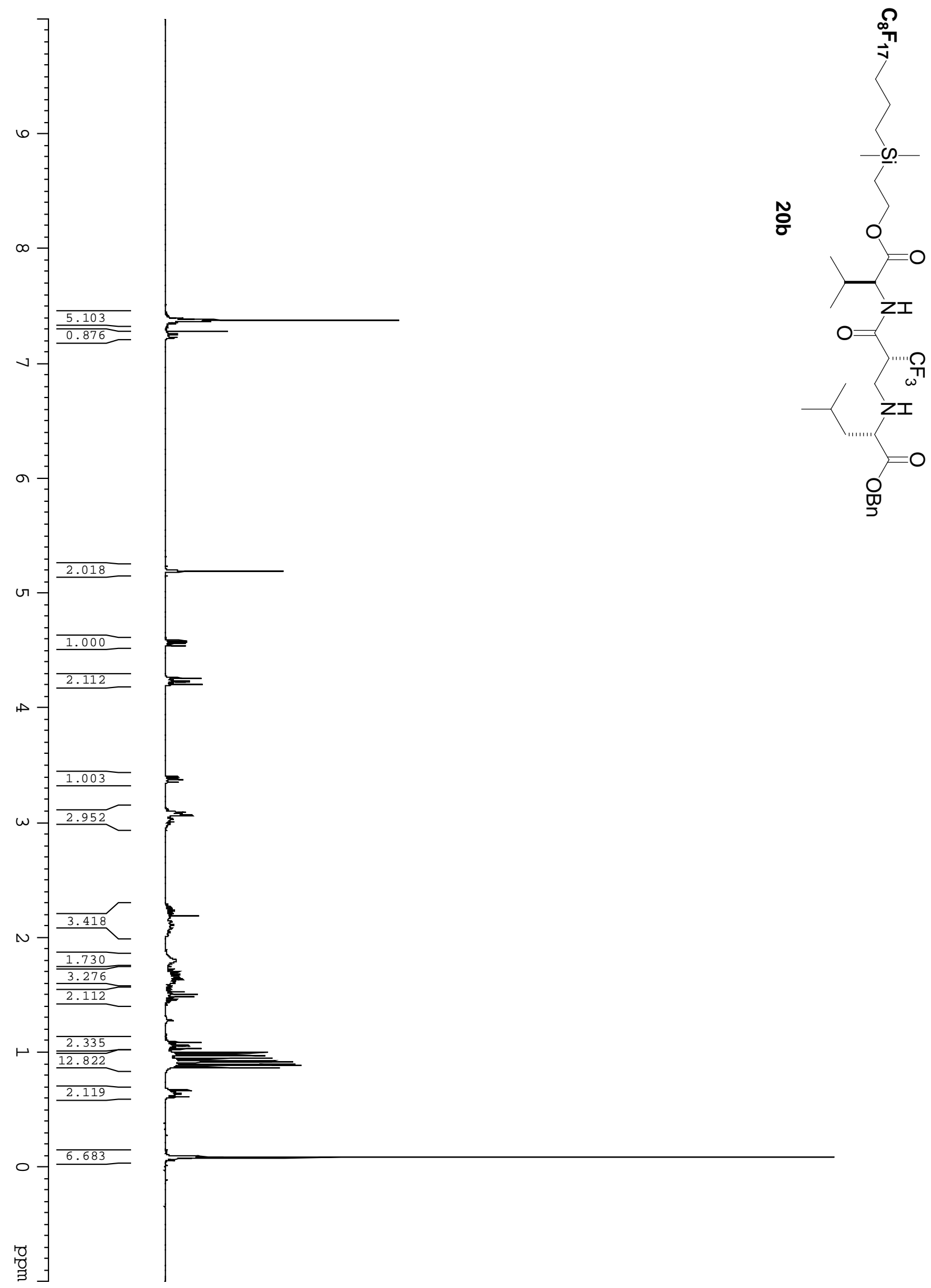




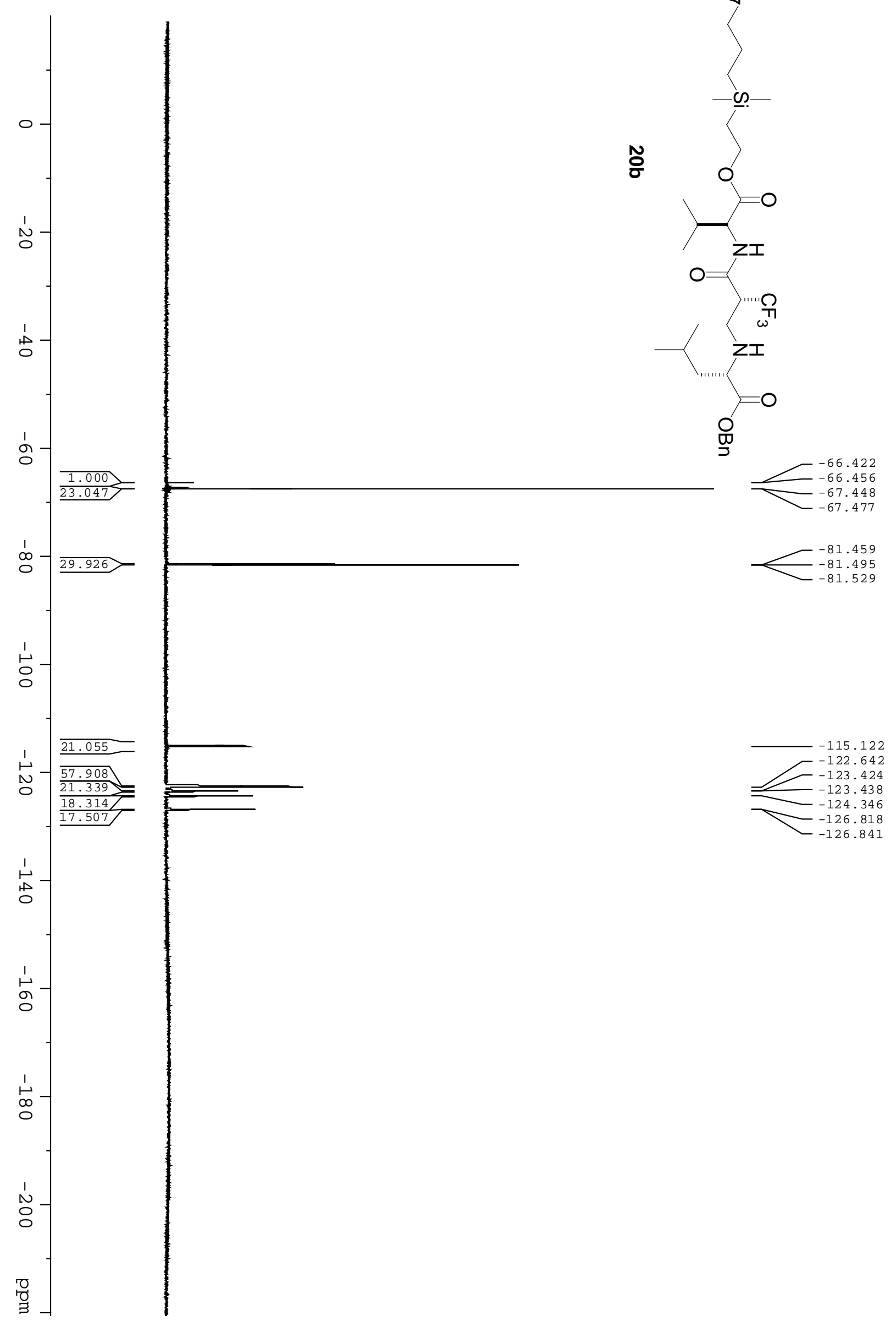




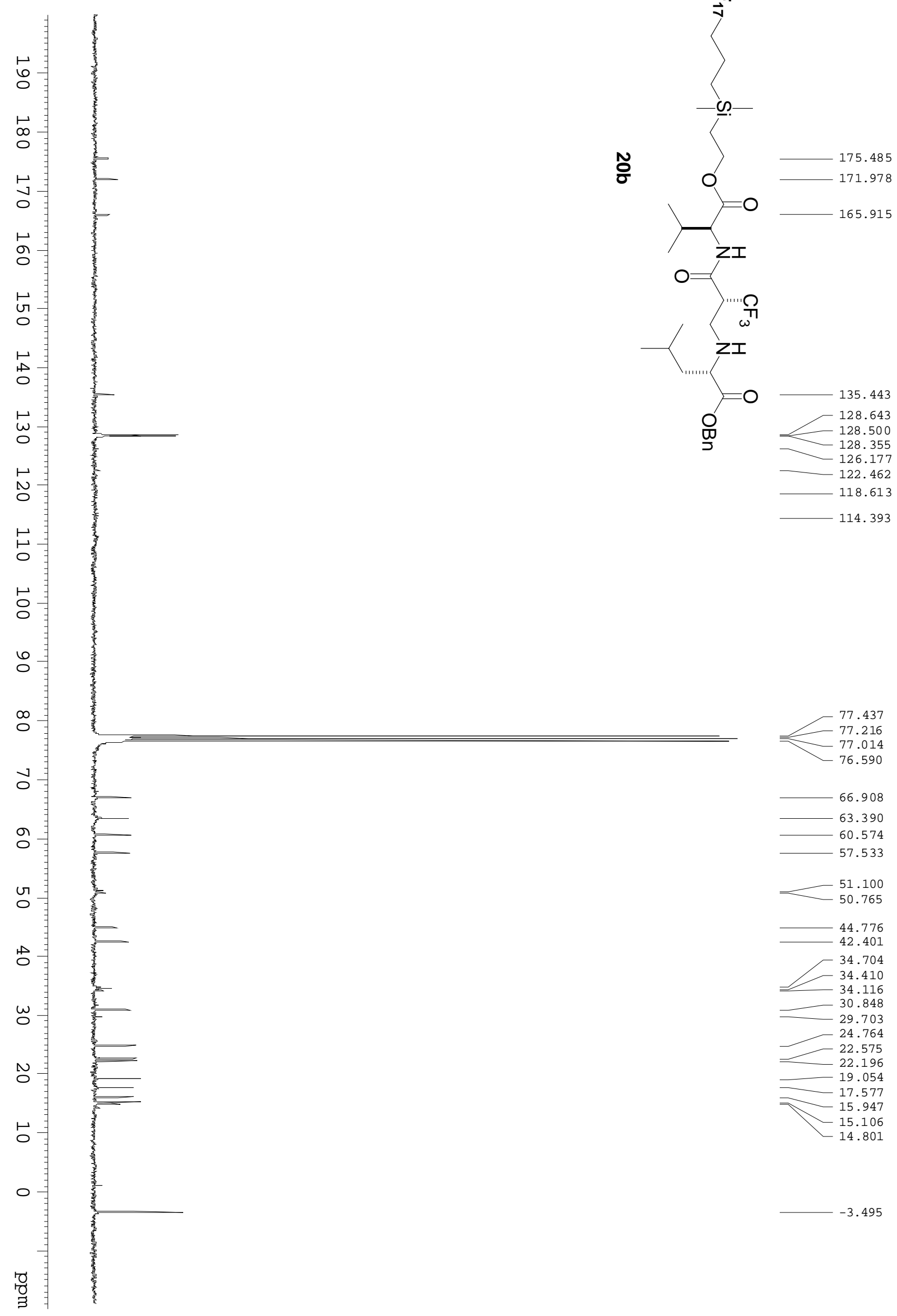




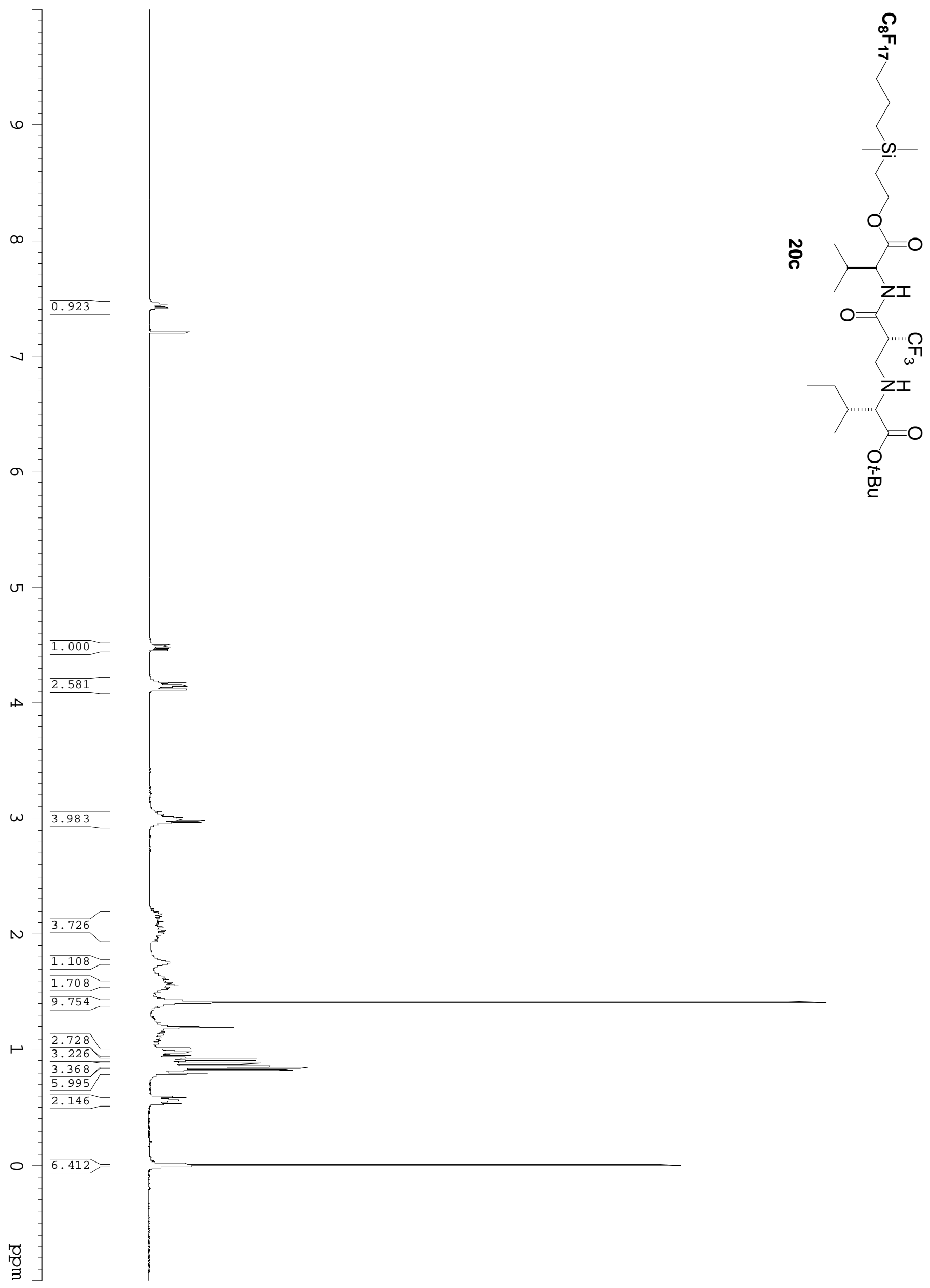




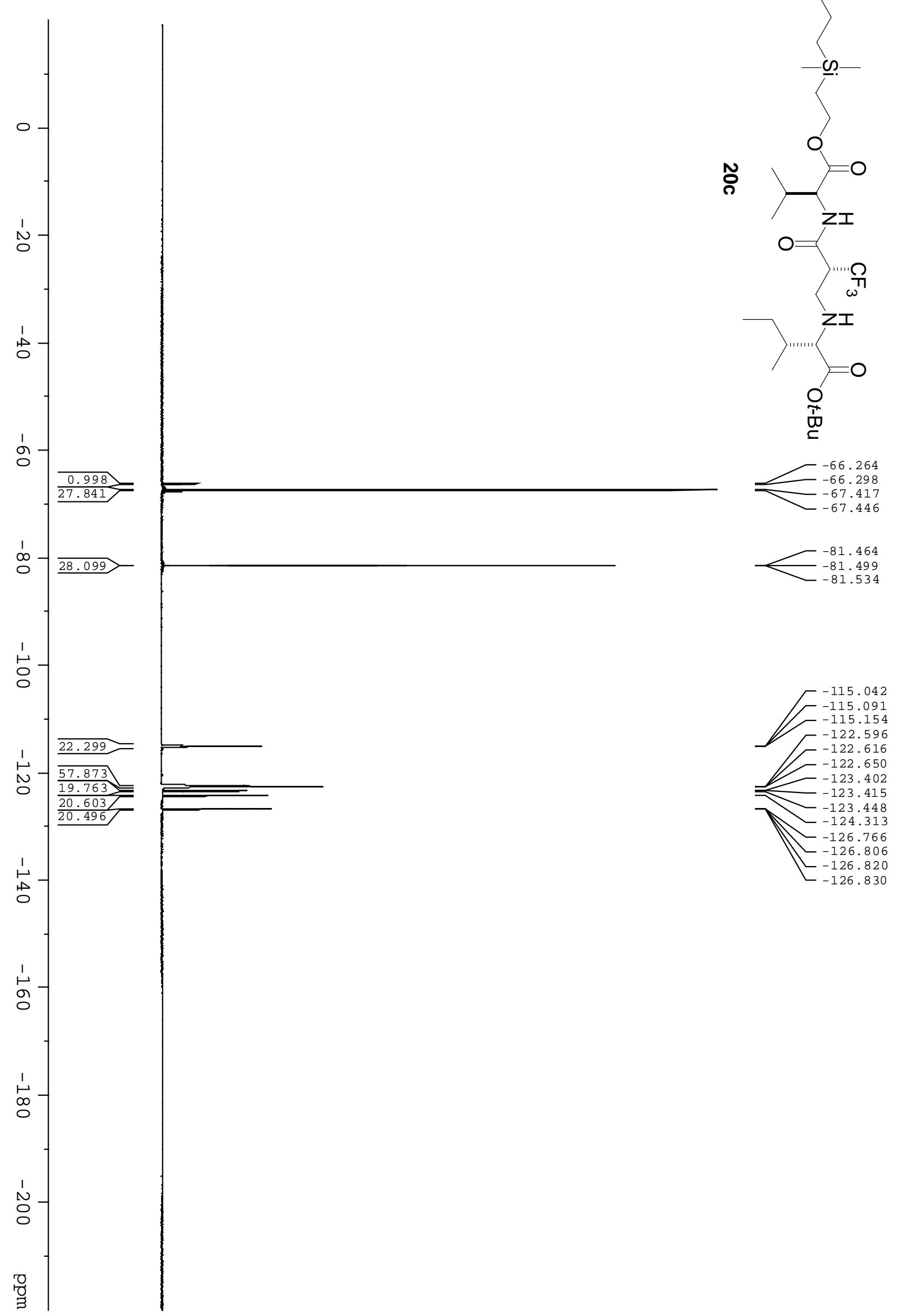




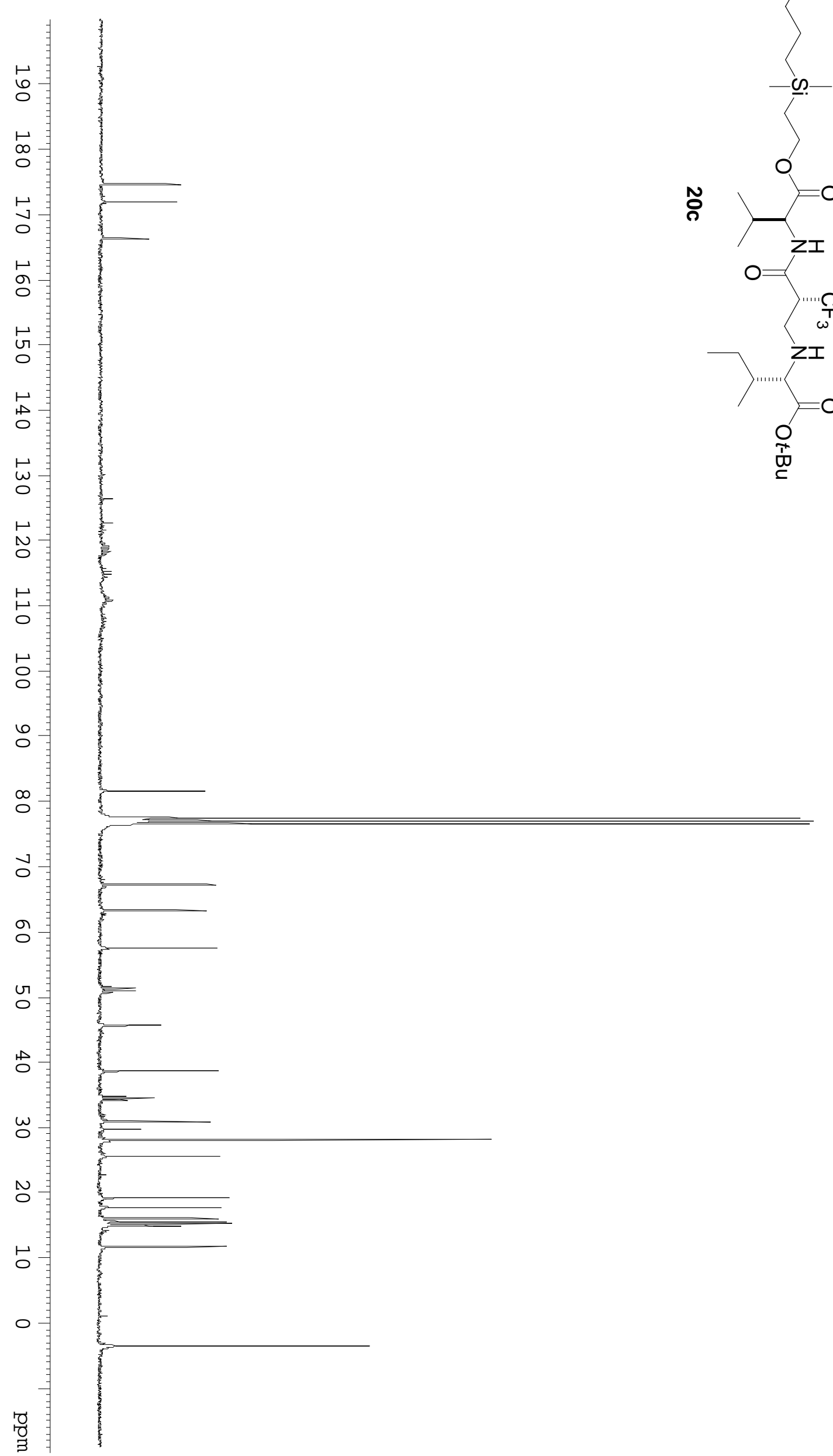

174.603 171.854 166. 277

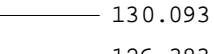
126.383 122.673 119.466 81.555 67.149 63.211 57.468 51.608

51.277 50.946 50.614 45.518 38.540 34.693 34.401 34.107 30.816 28.049 25.524 19.065 17.588 15.433 15.089 14.781 11.577 


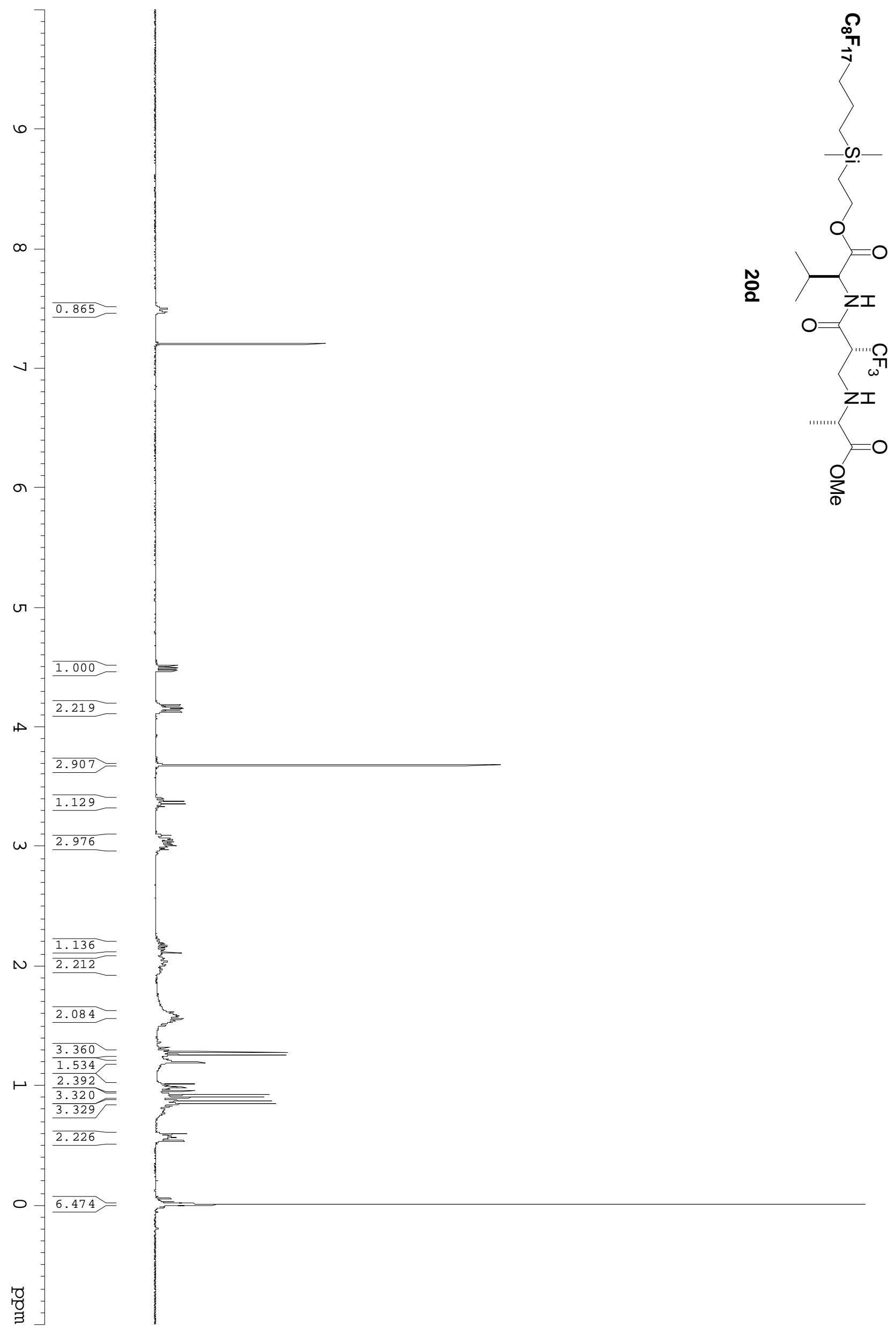




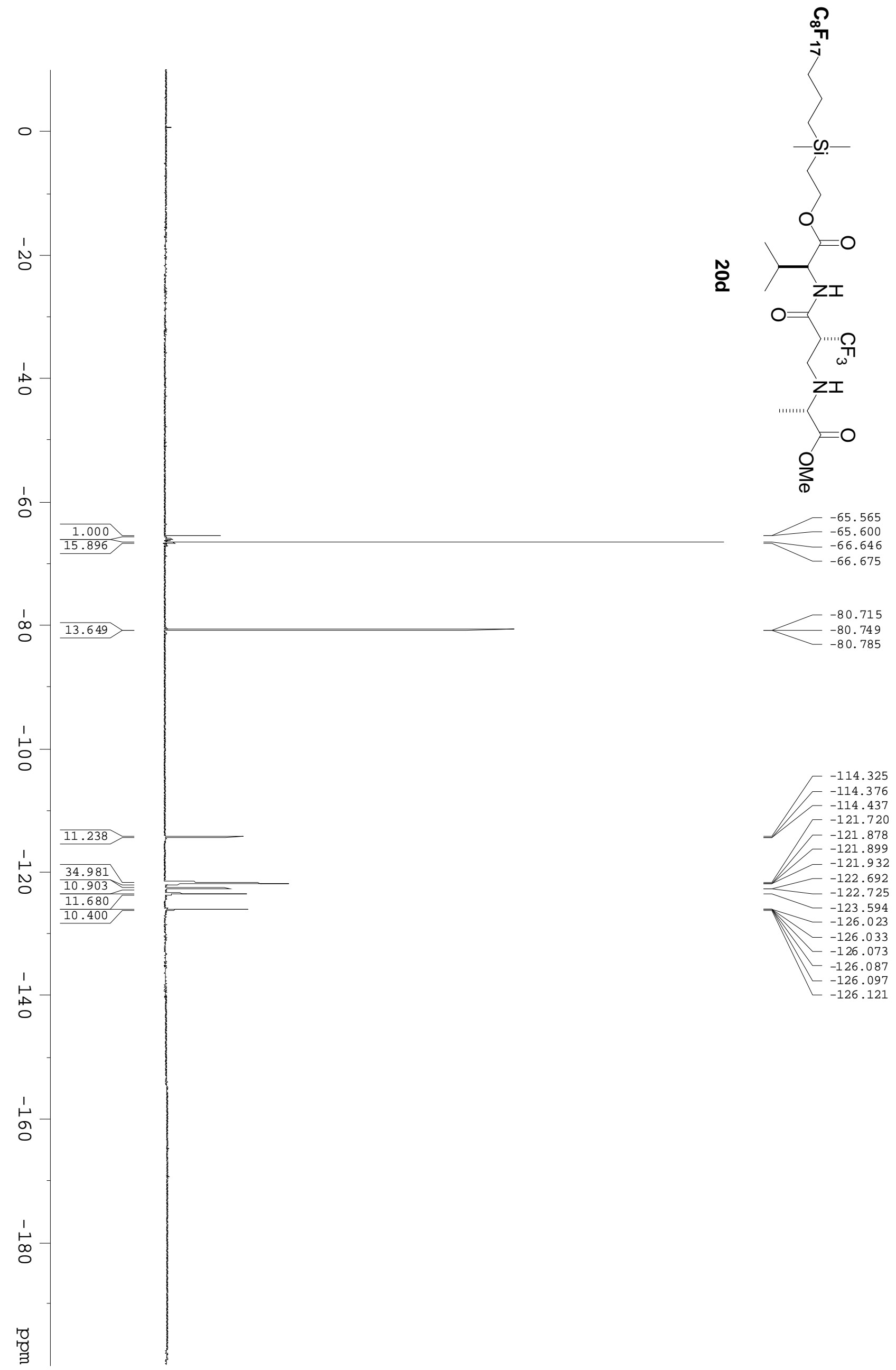



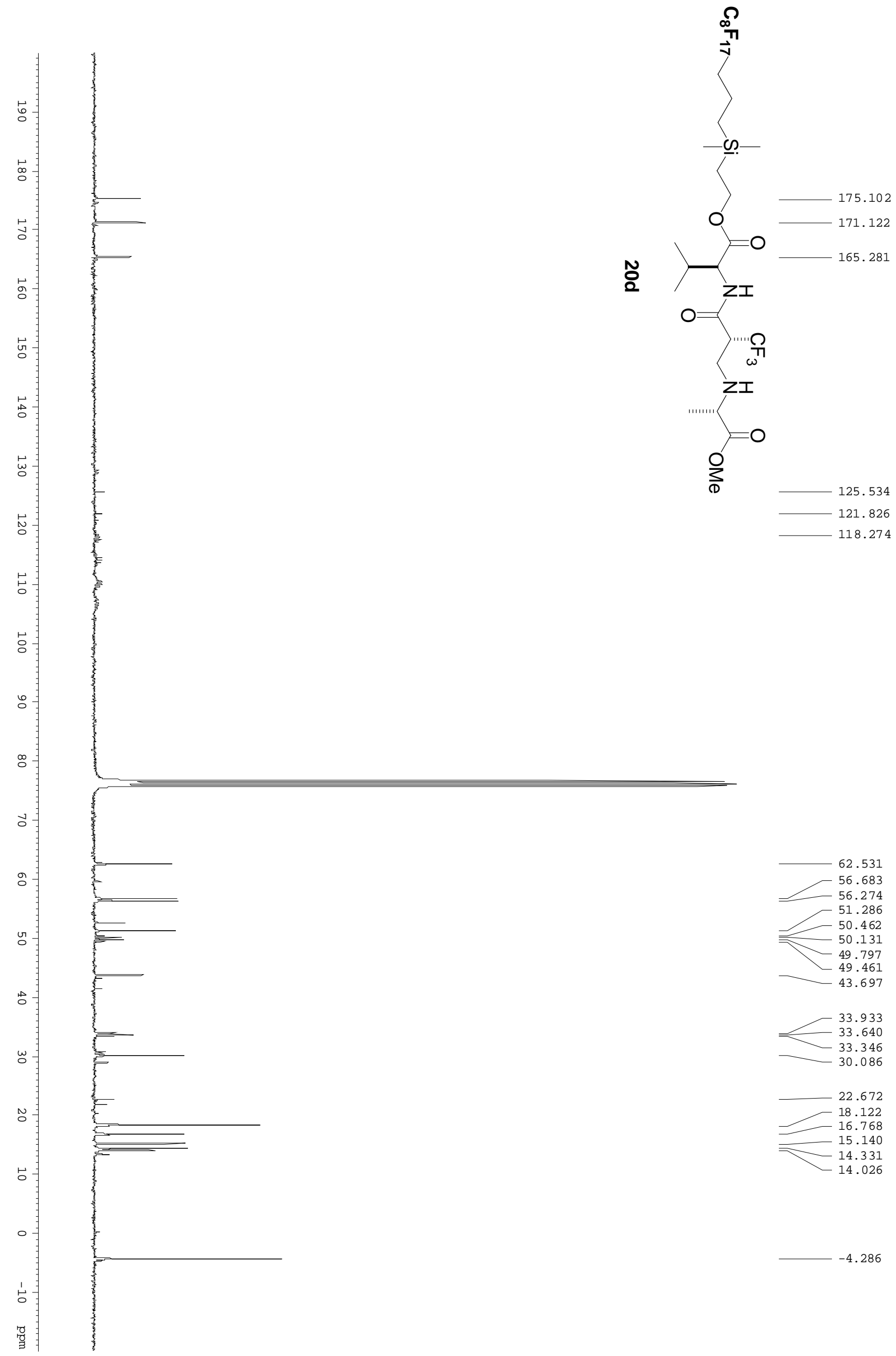

62.531

56.683

56.274

51.286

50.131

49.797
49.461

43.697

33.933

33.640

33.346

30.086

22.672

18.122

16.768

15.140

14.331

14.026

$-4.286$ 


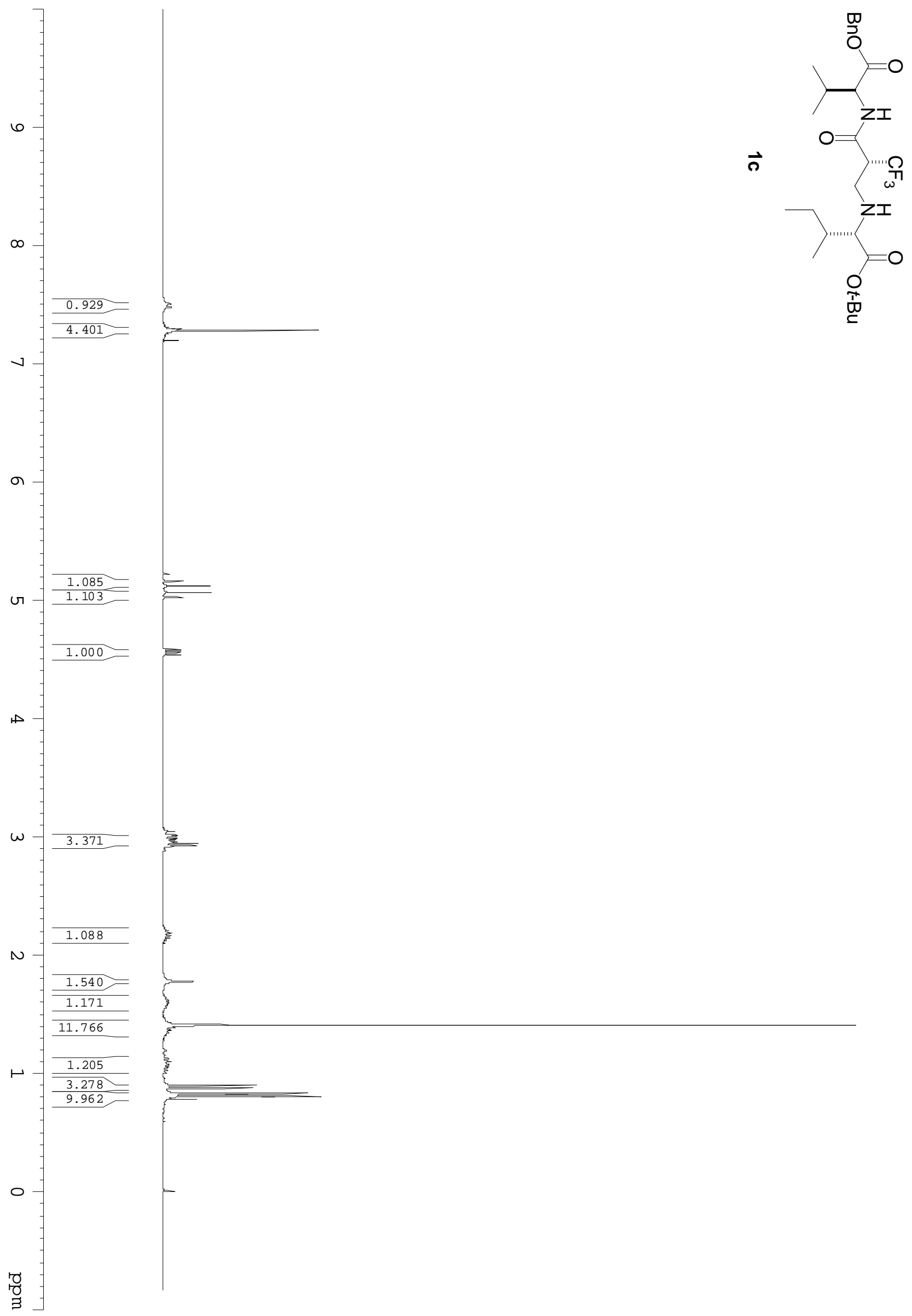




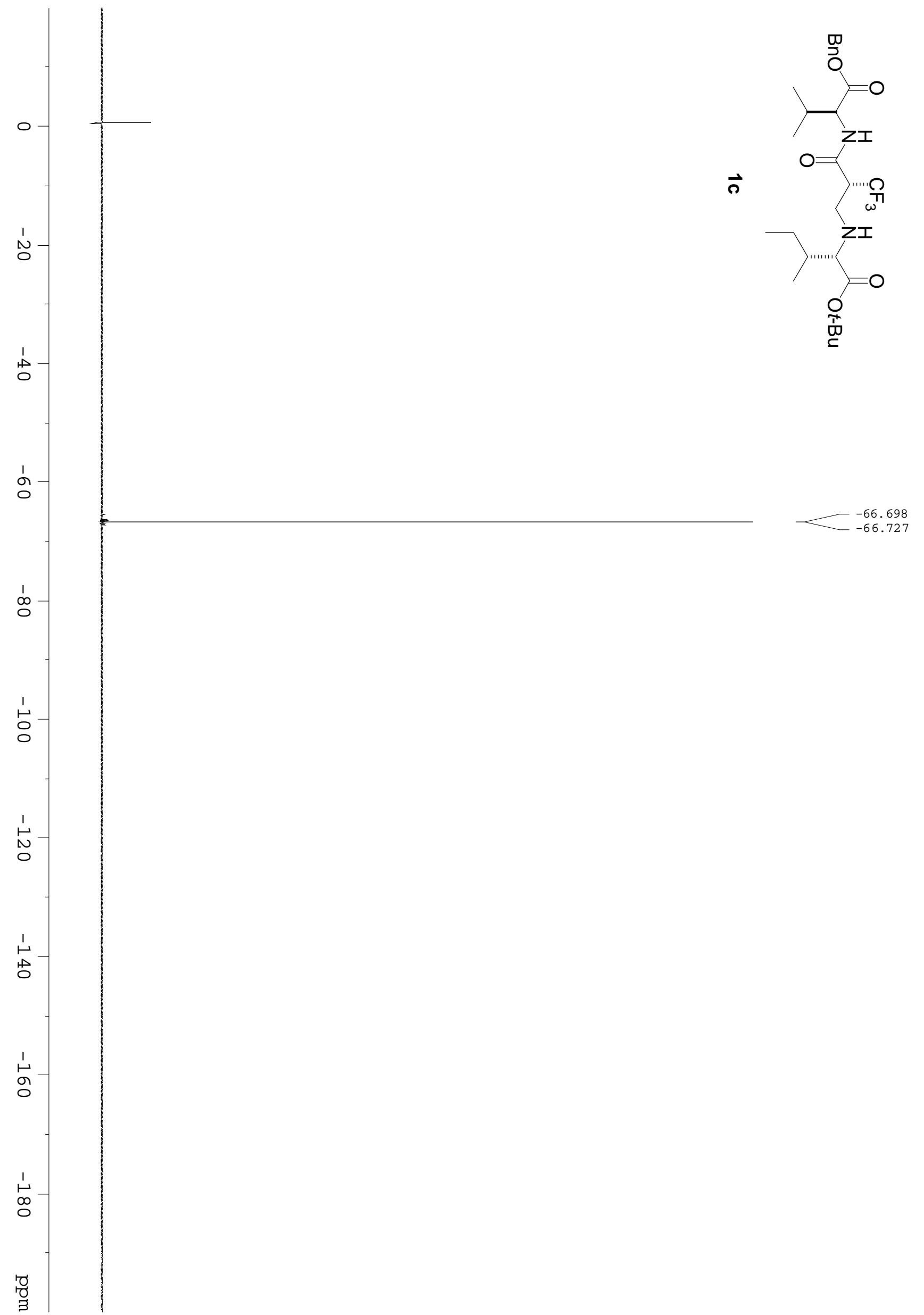




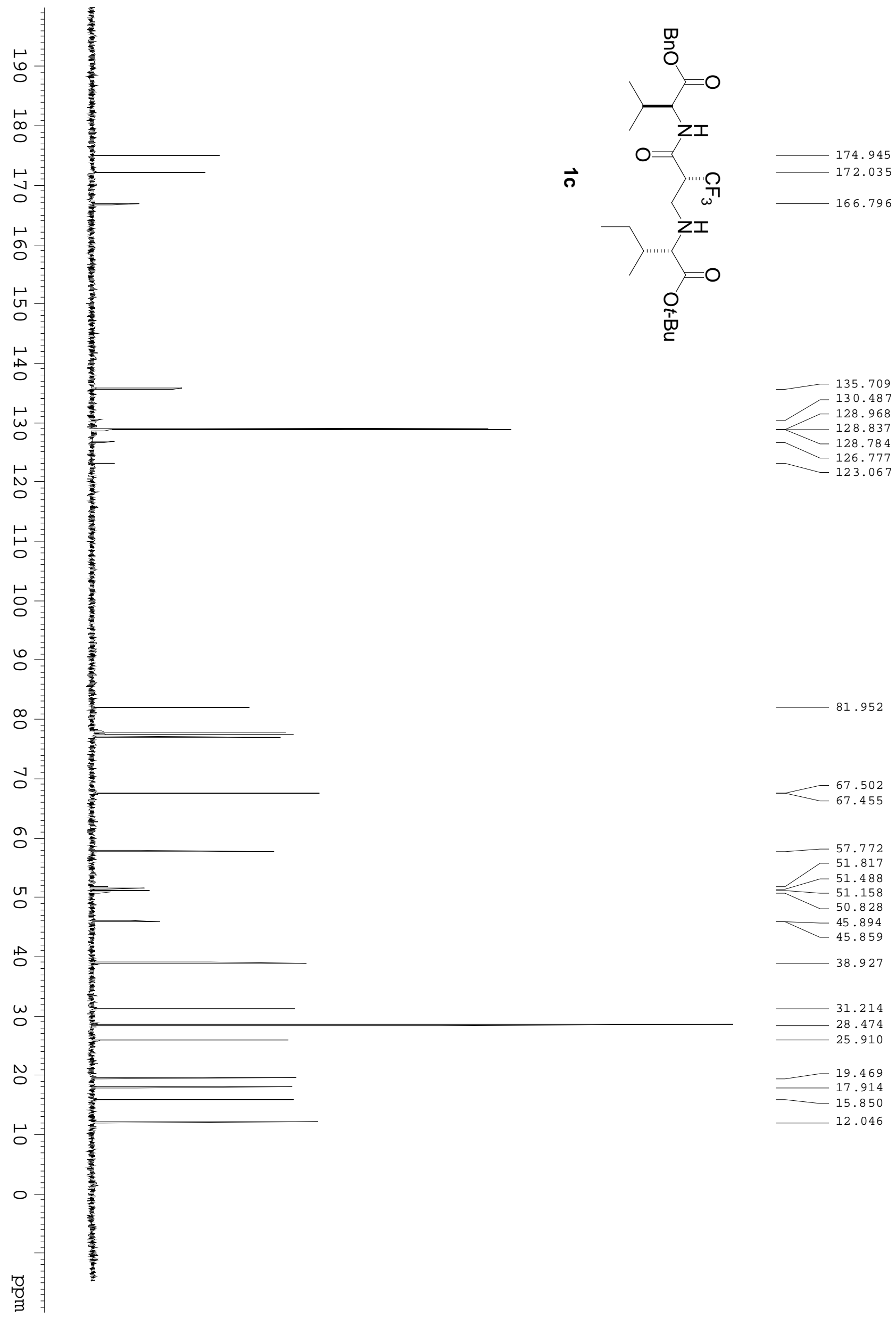

